\title{
Four-Dimensional Painlevé-Type Equations Associated with Ramified Linear Equations III: Garnier Systems and Fuji-Suzuki Systems
}

\author{
Hiroshi KAWAKAMI
}

College of Science and Engineering, Aoyama Gakuin University, 5-10-1 Fuchinobe, Chuo-ku, Sagamihara-shi, Kanagawa 252-5258, Japan

E-mail:kawakami@gem.aoyama.ac.jp

Received April 19, 2017, in final form December 07, 2017; Published online December 25, 2017 https://doi.org/10.3842/SIGMA.2017.096

\begin{abstract}
This is the last part of a series of three papers entitled "Four-dimensional Painlevé-type equations associated with ramified linear equations". In this series of papers we aim to construct the complete degeneration scheme of four-dimensional Painlevé-type equations. In the present paper, we consider the degeneration of the Garnier system in two variables and the Fuji-Suzuki system.
\end{abstract}

Key words: isomonodromic deformation; Painlevé equations; degeneration; integrable systems

2010 Mathematics Subject Classification: 34M55; 34M56; 33E17

\section{Introduction}

This is the last part of a series of three papers on four-dimensional Painlevé-type equations associated with ramified linear equations. By the term "Painlevé-type equations" we mean Hamiltonian systems which describe isomonodromic deformations of linear equations. The isomonodromic deformation is the deformation of a linear differential equation which do not change its "monodromy data" (see, for example, [9]), and it is known that isomonodromic deformation equations can be written in Hamiltonian form. In this terminology, the classical Painlevé equations are Painlevé-type equations with two-dimensional phase space.

The classical Painlevé equations are non-linear ordinary differential equations which were discovered by Painlevé [26] and Gambier [4]. Originally they were classified into six equations and are often denoted by $P_{\mathrm{I}}, P_{\mathrm{II}}, \ldots, P_{\mathrm{VI}}$. However, from a geometric viewpoint, it is natural to classify them into eight equations [27]. More precisely, the third Painlevé equation $P_{\mathrm{III}}$ is divided into three cases $P_{\mathrm{III}\left(D_{6}\right)}, P_{\mathrm{III}\left(D_{7}\right)}$, and $P_{\mathrm{III}\left(D_{8}\right)}$. The so-called third Painlevé equation is then $P_{\mathrm{III}\left(D_{6}\right)}$.

The eight Hamiltonians associated with the Painlevé equations are as follows [20, 22, 23, 24, 25]:

$$
\begin{aligned}
& t(t-1) H_{\mathrm{VI}}\left(\begin{array}{c}
\alpha, \beta \\
\gamma, \delta
\end{array} ; t ; q, p\right)=q(q-1)(q-t) p^{2} \\
& \quad+\{\delta q(q-1)-(2 \alpha+\beta+\gamma+\delta) q(q-t)+\gamma(q-1)(q-t)\} p+\alpha(\alpha+\beta)(q-t), \\
& t H_{\mathrm{V}}\left(\begin{array}{c}
\alpha, \beta \\
\gamma
\end{array} ; t ;, p\right)=p(p+t) q(q-1)+\beta p q+\gamma p-(\alpha+\gamma) t q, \\
& H_{\mathrm{IV}}(\alpha, \beta ; t ; q, p)=p q(p-q-t)+\beta p+\alpha q, \\
& t H_{\mathrm{III}\left(D_{6}\right)}(\alpha, \beta ; t ; q, p)=p^{2} q^{2}-\left(q^{2}-\beta q-t\right) p-\alpha q, \\
& t H_{\mathrm{III}\left(D_{7}\right)}(\alpha ; t ; q, p)=p^{2} q^{2}+\alpha q p+t p+q,
\end{aligned}
$$




$$
\begin{aligned}
& t H_{\mathrm{III}\left(D_{8}\right)}(t ; q, p)=p^{2} q^{2}+q p-q-\frac{t}{q}, \\
& H_{\mathrm{II}}(\alpha ; t ; q, p)=p^{2}-\left(q^{2}+t\right) p-\alpha q, \\
& H_{\mathrm{I}}(t ; q, p)=p^{2}-q^{3}-t q .
\end{aligned}
$$

The standard linear equations associated with the classical Painlevé equations are given by certain second order single linear equations, or equivalently, by first order $2 \times 2$ systems. Here we review the classification of the classical Painlevé equations in terms of associated linear equations.

It is well-known that the classical Painlevé equations admit degeneration. We use the term degeneration in the following sense. Suppose a differential equation $E$ has some parameter $\varepsilon$. When the equation $E$ tends to another equation $E^{\prime}$ as $\varepsilon$ tends to 0 , we say that $E$ degenerates to $E^{\prime}$. The following scheme is the well-known degeneration scheme among the six Painlevé equations:

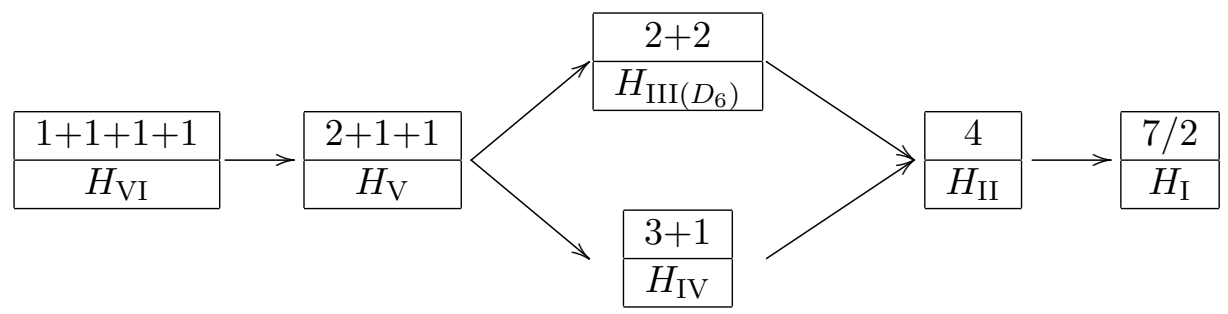

The number in each box is the "singularity pattern" of an associated linear equation, which has information on the Paincaré ranks of the singular points of the linear equation. In this case, the linear equations are well characterized by their singularity pattern.

Here we point out that

- this scheme lacks the third Painlevé equations of type $D_{7}^{(1)}$ and $D_{8}^{(1)}$,

- from the viewpoint of associated linear equations, the degeneration $H_{\mathrm{II}} \rightarrow H_{\mathrm{I}}$ is distinguished from the others. Namely, the other degenerations correspond to the "confluence of singular points", while the degeneration $H_{\mathrm{II}} \rightarrow H_{\mathrm{I}}$ corresponds to the "degeneration of an HTL canonical form".

We note that there are two kinds of degenerations concerning linear equations: confluence of singular points and degeneration of an HTL canonical form (where HTL is an abbreviation for Hukuhara-Turrittin-Levelt).

By considering all the possible degenerations of HTL canonical forms (and possible confluences), one can obtain the following complete degeneration scheme of the classical Painlevé equations $[10,11,21]$.

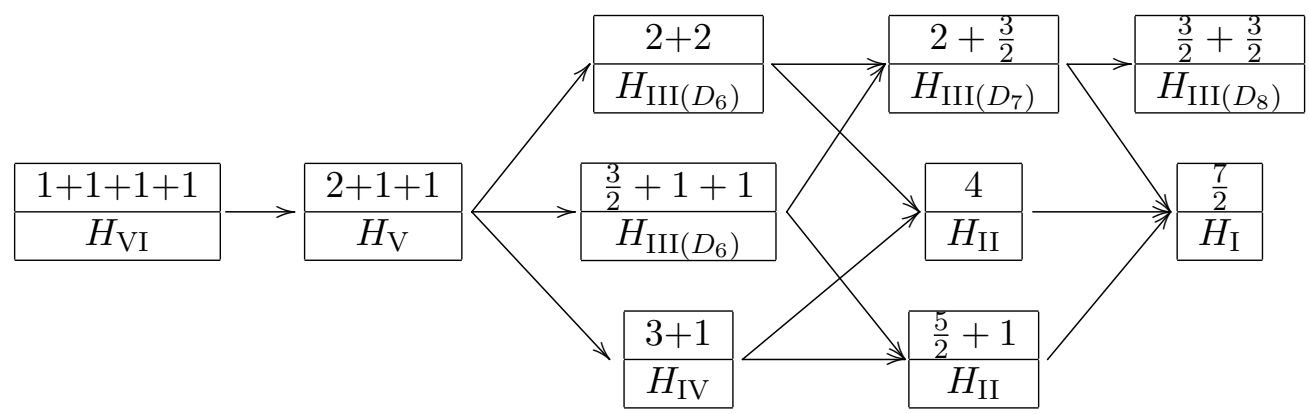

Thus we can say that the sixth Painlevé equation is the "master equation" from which we can derive all the other Painlevé equations by successive degenerations. 
Recently, many generalizations of the Painlevé equations have been discovered. What is important here is that they can be written as compatibility conditions of linear differential equations. Thus they can be regarded as isomonodromic deformation equations of some linear differential equations.

The purpose of our study is to understand those of four-dimensional phase space using associated linear equations. More specifically, we construct the degeneration scheme starting from suitable Painlevé-type equations so that we can provide a unified perspective of four-dimensional analogues of the Painlevé equations.

In [15], the degeneration scheme of four-dimensional Painlevé-type equations associated with unramified linear equations was obtained. They considered degenerations from the following four Painlevé-type equations:

$$
H_{\mathrm{Gar}}^{1+1+1+1+1}, \quad H_{\mathrm{FS}}^{A_{5}}, \quad H_{\mathrm{Ss}}^{D_{6}}, \quad H_{\mathrm{VI}}^{\mathrm{Mat}} .
$$

These stand for the Hamiltonians for the four-dimensional Painlevé-type equations: the Garnier system in two variables [5], the Fuji-Suzuki system [3, 31], the Sasano system [29], and the sixth matrix Painlevé system [2, 12, 15]. They correspond to four Fuchsian systems characterized by the following spectral types:

$$
11,11,11,11,11 \quad 21,21,111,111 \quad 31,22,22,1111 \quad 22,22,22,211
$$

respectively. The above four Painlevé-type equations are the master equations in four-dimensional case. We note that the degenerations considered in the paper correspond to "confluence of singular points" of linear equations.

The aim of the present series of papers is to obtain the "complete" degeneration scheme of the Painlevé-type equations with four-dimensional phase space. In order to achieve this goal, we have to consider the degeneration of HTL canonical forms in four-dimensional case. Note that, unlike the two-dimensional case, degenerations of HTL canonical forms treated in this study do not necessarily correspond to degenerations of Jordan canonical forms (see Section 2.4 and [14]).

In Part I and Part II [13, 14] we consider degenerations from the 22, 22, 22, 211-system and the 31,22,22,1111-system. These correspond to the degeneration of the sixth matrix Painlevé system and the Sasano system, respectively.

In this Part III, we treat the degeneration of the Garnier system in two variables and what we call the Fuji-Suzuki system.

Note that the degeneration scheme of the Garnier system corresponding to confluences of singular points (and a degeneration of an HTL canonical form) was already obtained by Kimura [17]. The complete list of degenerate Garnier systems associated with ramified linear equations was obtained by Kawamuko [16]. In this paper, we recalculated the Hamiltonians and Lax pairs for the degenerate Garnier systems.

This paper is organized as follows. In Section 2 we recall the notions of HTL canonical forms, Riemann schemes, singularity patterns, and spectral types. We also discuss the degeneration of HTL canonical forms. In Section 3 we present the linear equations of ramified type which can be obtained by degeneration from the 11,11,11,11,11-system and the 21,21,111,111-system and the corresponding Hamiltonians. In Section 4 we discuss correspondences of linear systems through the Laplace transform. In Section 5 we give the degeneration scheme of the Hamiltonians of four-dimensional Painlevé-type equations. In the Appendix we give data on degenerations.

Here we present the degeneration scheme of the Garnier system and the Fuji-Suzuki system, which is the main result of this paper. 


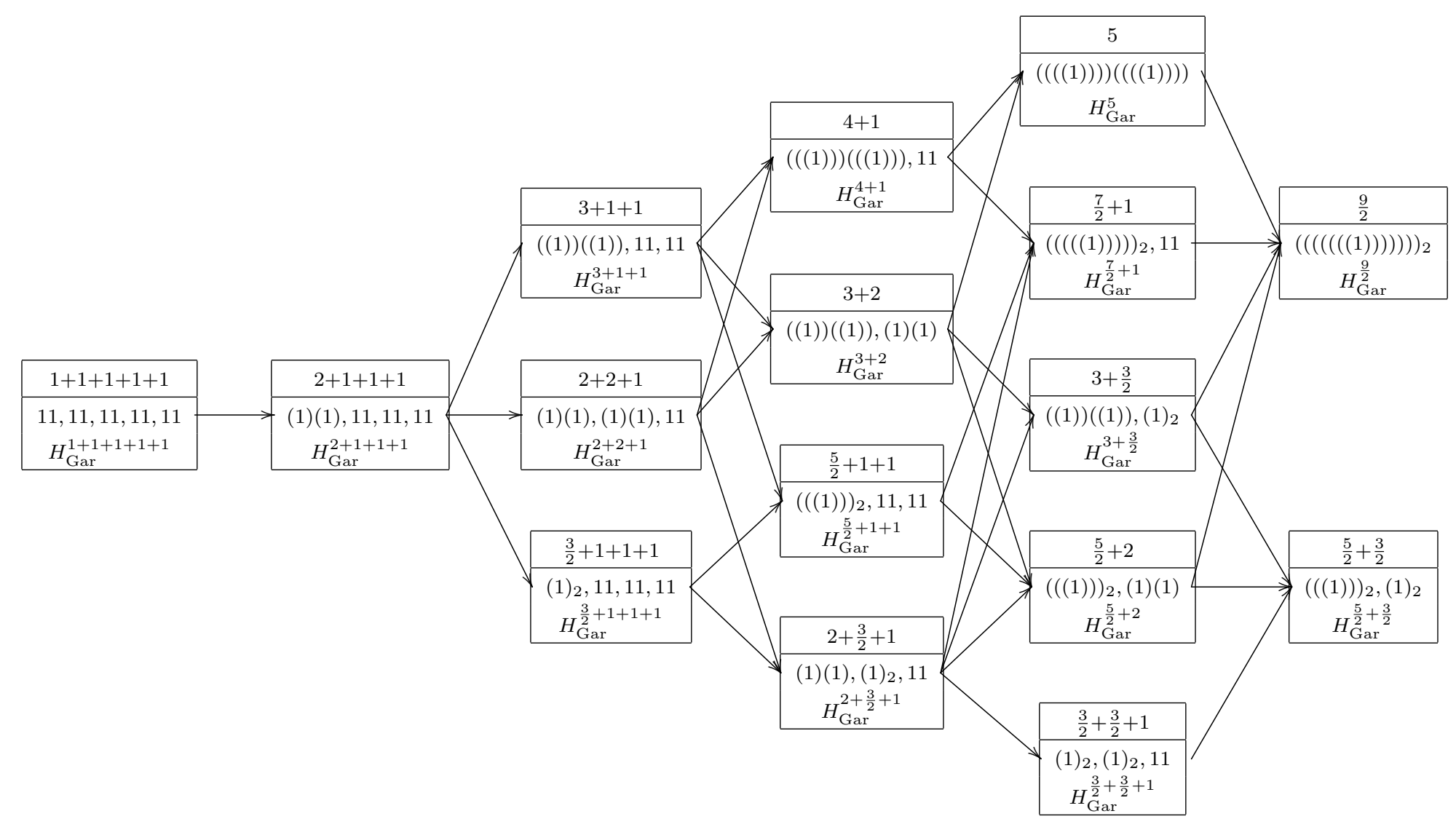




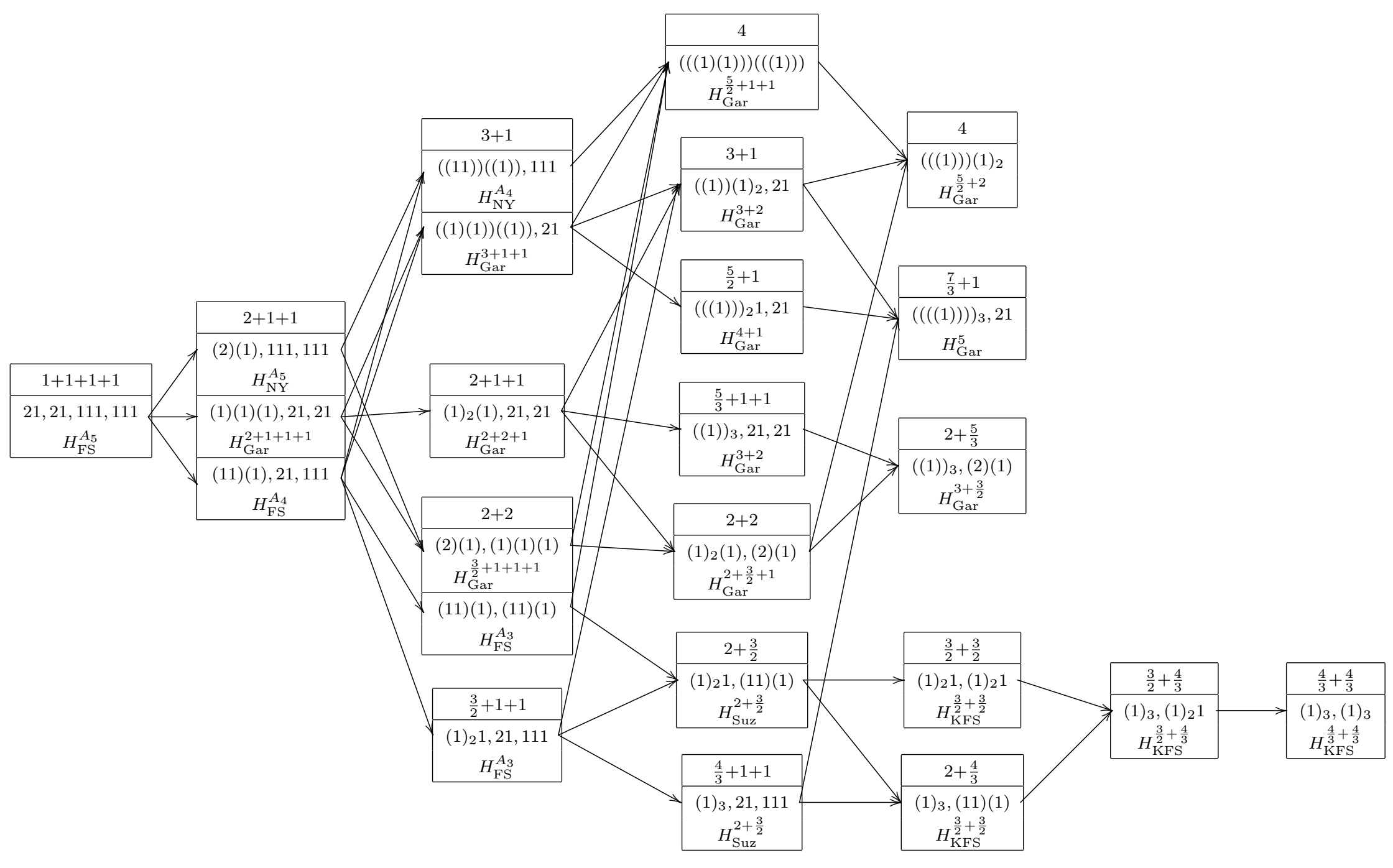


Symbols such as $H_{\mathrm{Gar}}^{2+1+1+1}$ stand for the Hamiltonians for four-dimensional Painlevé-type equations. The explicit expressions for them are given in Section 3.

\section{Notions on linear differential equations}

In this section we recall some notions on linear differential equations.

\subsection{HTL canonical forms}

Consider a system of linear differential equations

$$
\frac{\mathrm{d} Y}{\mathrm{~d} x}=A(x) Y
$$

A change of the dependent variable $Y=P Z$ with an invertible matrix $P=P(x)$ yields the following system:

$$
\frac{\mathrm{d} Z}{\mathrm{~d} x}=\left(P^{-1} A(x) P-P^{-1} P^{\prime}\right) Z .
$$

The transformation

$$
A(x) \mapsto A^{P}(x):=P^{-1} A(x) P-P^{-1} P^{\prime}
$$

is called the gauge transformation by $P$.

A system of linear differential equations with rational function coefficients

$$
\frac{\mathrm{d} Y}{\mathrm{~d} x}=\left(\sum_{\nu=1}^{n} \sum_{k=0}^{r_{\nu}} \frac{A_{\nu}^{(k)}}{\left(x-u_{\nu}\right)^{k+1}}+\sum_{k=1}^{r_{\infty}} A_{\infty}^{(k)} x^{k-1}\right) Y, \quad A_{*}^{(k)} \in M(m, \mathbb{C})
$$

can be transformed into the "canonical form" at each singular point.

The system (2.1) has singularity at $x=u_{\nu}(\nu=1, \ldots, n)$ and $x=u_{0}:=\infty$. Set $z=x-u_{\nu}$ $(\nu=1, \ldots, n)$ or $z=1 / x$. We consider the system around $z=0$ :

$$
\frac{\mathrm{d} Y}{\mathrm{~d} z}=\left(\frac{A_{0}}{z^{r+1}}+\frac{A_{1}}{z^{r}}+\cdots+A_{r+1}+A_{r+2} z+\cdots\right) Y .
$$

Let $\mathcal{P}_{z}=\cup_{p>0} \mathbb{C}\left(\left(z^{1 / p}\right)\right)$ be the field of Puiseux series where $\mathbb{C}((t))$ is the field of formal Laurent series in $t$.

Definition 2.1 (HTL canonical form). An element

$$
\frac{D_{0}}{z^{l_{0}}}+\frac{D_{1}}{z^{l_{1}}}+\cdots+\frac{D_{s-1}}{z^{l_{s-1}}}+\frac{\Theta}{z^{l_{s}}}
$$

in $M_{m}\left(\mathcal{P}_{z}\right)$ satisfying the following conditions:

- $l_{j}$ is a rational number, $l_{0}>l_{1}>\cdots>l_{s-1}>l_{s}=1$,

- $D_{0}, \ldots, D_{s-1}$ are commuting diagonalizable matrices,

- $\Theta$ is a (not necessarily diagonalizable) matrix that commutes with all $D_{j}$ 's

is called an HTL canonical form, or HTL form for short. 
Theorem 2.2 (Hukuhara [8], Levelt [18], Turrittin [32]). For any

$$
A(z)=\frac{A_{0}}{z^{r+1}}+\frac{A_{1}}{z^{r}}+\cdots \in M_{m}(\mathbb{C}((z))),
$$

there exists $P \in \mathrm{GL}\left(m, \mathcal{P}_{z}\right)$ such that $A^{P}(z)$ is an $H T L$ form

$$
\frac{D_{0}}{z^{l_{0}}}+\frac{D_{1}}{z^{l_{1}}}+\cdots+\frac{D_{s-1}}{z^{l_{s-1}}}+\frac{\Theta}{z} .
$$

Here $l_{0}, \ldots, l_{s-1}$ are uniquely determined only by $A(z)$.

If

$$
\frac{\tilde{D}_{0}}{z^{l_{0}}}+\frac{\tilde{D}_{1}}{z^{l_{1}}}+\cdots+\frac{\tilde{D}_{s-1}}{z^{l_{s-1}}}+\frac{\tilde{\Theta}}{z}
$$

is another HTL form of the same $A(z)$, then there exist $g \in \mathrm{GL}(m, \mathbb{C})$ and $k \in \mathbb{Z}_{\geq 1}$ such that

$$
\tilde{D}_{j}=g^{-1} D_{j} g, \quad \exp (2 \pi i k \tilde{\Theta})=g^{-1} \exp (2 \pi i k \Theta) g
$$

hold.

There is an algorithm for constructing the HTL forms of a given linear system. We will briefly explain how to construct HTL forms in Section 2.3.

The number $l_{0}-1$ is called the Poincaré rank of the singular point. If there is a rational number $l_{j} \in \mathbb{Q} \backslash \mathbb{Z}$, the singular point is called a ramified irregular singular point. A linear equation is said to be of ramified type if the equation has a ramified irregular singular point.

When we roughly express the singularity of a linear differential equation, we attach the number "Poincaré rank +1 " to each singular point and connect the numbers with "+". We call it the singularity pattern of the equation.

\section{$2.2 \quad$ Riemann schemes}

Consider an HTL form

$$
\frac{D_{0}}{z^{l_{0}}}+\frac{D_{1}}{z^{l_{1}}}+\cdots+\frac{D_{s-1}}{z^{l_{s-1}}}+\frac{\Theta}{z} .
$$

Here we assume $D_{j}$ 's and $\Theta$ are in Jordan canonical form.

Let $d \in \mathbb{Z}_{>0}$ be the minimum element of

$$
\left\{k \in \mathbb{Z}_{>0} \mid k l_{j} \in \mathbb{Z}(j=0, \ldots, s-1)\right\} .
$$

Notice that $d=1$ is equivalent to the unramifiedness of the singular point. Then the HTL form (2.2) can be rewritten as

$$
\frac{T_{0}}{z^{\frac{b}{d}+1}}+\frac{T_{1}}{z^{\frac{b-1}{d}+1}}+\cdots+\frac{T_{b-1}}{z^{\frac{1}{d}+1}}+\frac{\Theta}{z}
$$

where $l_{0}=\frac{b}{d}+1$.

When $\Theta$ is a diagonal matrix, we denote this HTL form by

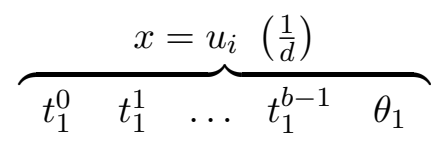

$$
\begin{aligned}
& \begin{array}{ccccc}
\vdots & \vdots & & \vdots & \vdots \\
t_{m}^{0} & t_{m}^{1} & \ldots & t_{m}^{b-1} & \theta_{m}
\end{array}
\end{aligned}
$$

where $T_{j}=\operatorname{diag}\left(t_{1}^{j}, \ldots, t_{m}^{j}\right), \Theta=\operatorname{diag}\left(\theta_{1}, \ldots, \theta_{m}\right)$. In the case of $d=1$, we omit $\left(\frac{1}{d}\right)$. 
Remark 2.3. In this series of papers, the matrix $\Theta$ is always diagonalizable.

The table of the HTL forms (represented by the above formula) at all singular points is called the Riemann scheme of a linear equation.

Concerning the computation of HTL forms, the following two theorems are fundamental.

Theorem 2.4 ([33]). For any

$$
A(z)=\frac{1}{z}\left(A_{0}+A_{1} z+\cdots\right)
$$

there exists a formal power series $P=P(z)$ such that

$$
A^{P}(z)=\frac{\tilde{A}_{0}}{z}
$$

When no two different eigenvalues of $A_{0}$ differ by an integer, we can choose the gauge so that $\tilde{A}_{0}=A_{0}$.

Theorem 2.5 (block diagonalization [33]). Let $A(z)$ be

$$
A(z)=\frac{1}{z^{r+1}}\left(A_{0}+A_{1} z+\cdots\right), \quad r \in \mathbb{Z}_{>0},
$$

where the eigenvalues of $A_{0}$ are assumed to be $\lambda_{1}, \ldots, \lambda_{n}$. Without loss of generality, we can assume that $A_{0}$ is in Jordan canonical form

$$
A_{0}=J_{1}\left(\lambda_{1}\right) \oplus \cdots \oplus J_{n}\left(\lambda_{n}\right)
$$

where $J_{k}\left(\lambda_{k}\right)$ is a direct sum of Jordan blocks with the eigenvalue $\lambda_{k}$.

Then there exists a formal power series $P=P(z)$ such that

$$
A^{P}(z)=B_{1}(z) \oplus \cdots \oplus B_{n}(z), \quad B_{k}(z)=\frac{1}{z^{r+1}}\left(B_{0}^{k}+B_{1}^{k} z+\cdots\right),
$$

where $B_{0}^{k}=J_{k}\left(\lambda_{k}\right)$.

\section{$2.3 \quad$ Spectral types}

We have introduced the notion of singularity pattern to represent the singularity of a linear system. However, if the rank of a linear system is greater than two, the singularity pattern is too rough to describe the singularity of the linear system sufficiently since it only has information of the Poincaré ranks.

In order to describe the singularity of a linear system in more detail, we need the notion of spectral type of linear equations.

Spectral types are defined through HTL forms.

- In the unramified case, the spectral type is defined to be the (tuple of) "refining sequence of partitions (RSP)".

- In the ramified case, the spectral type consists of "copies" of RSPs.

Thus we begin with the unramified case. 
2.3.1 Spectral types of unramified irregular singularities

Consider a system of linear differential equations whose coefficient matrix is

$$
A(z)=\frac{1}{z^{r+1}}\left(A_{0}+A_{1} z+\cdots\right), \quad r \in \mathbb{Z}_{>0} .
$$

Now suppose that $A_{0}$ is diagonalizable. Applying the block diagonalization (see Theorem 2.5) to $(2.3)$, we see that the leading terms $B_{0}^{k}(k=1, \ldots, n)$ are all scalar matrices.

We focus on the next $B_{1}^{k}$ in each block. Suppose that all the $B_{1}^{k}(k=1, \ldots, n)$ are also diagonalizable. Then diagonalize them by constant gauge transformations and apply Theorem 2.5 to each block. Thus each block decomposes into smaller direct summands again. We note that the first two terms of each direct summands thus obtained are scalar matrices.

In general, if $B_{r-j+1}$ is diagonalizable in a direct summand

$$
\frac{c_{0} I}{z^{r+1}}+\frac{c_{1} I}{z^{r}}+\cdots+\frac{c_{r-j} I}{z^{j+1}}+\frac{B_{r-j+1}}{z^{j}}+\cdots
$$

then, by a gauge transformation, we have

$$
\sim \frac{c_{0} I}{z^{r+1}}+\frac{c_{1} I}{z^{r}}+\cdots+\frac{c_{r-j} I}{z^{j+1}}+\frac{D_{r-j+1}}{z^{j}}+\cdots
$$

where $D_{r-j+1}=d_{1} I_{m_{1}} \oplus \cdots \oplus d_{l} I_{m_{l}}$. After the block diagonalization with respect to the eigenvalues of $D_{r-j+1}$, the above series decomposes into smaller direct summands

$$
\frac{c_{0} I_{m_{i}}}{z^{r+1}}+\frac{c_{1} I_{m_{i}}}{z^{r}}+\cdots+\frac{c_{r-j} I_{m_{i}}}{z^{j+1}}+\frac{d_{i} I_{m_{i}}}{z^{j}}+\frac{*}{z^{j-1}}+\cdots, \quad i=1 \ldots, l .
$$

By repeating the above procedure, we can decompose (2.3) into a direct sum of the series of the following form

$$
\frac{c_{0} I}{z^{r+1}}+\frac{c_{1} I}{z^{r}}+\cdots+\frac{c_{r-1} I}{z^{2}}+\frac{C_{r}}{z}+\cdots
$$

By virtue of Theorem 2.4, we can eliminate all the terms with non-negative powers of $z$ by a suitable gauge transformation. As the result, (2.3) transforms into a direct sum of matrices of the following form

$$
\frac{c_{0} I}{z^{r+1}}+\frac{c_{1} I}{z^{r}}+\cdots+\frac{c_{r-1} I}{z^{2}}+\frac{\tilde{C}_{r}}{z}
$$

The direct sum of the above forms thus obtained is the HTL canonical form.

In this case (i.e., the unramified case), by construction, the feature of an HTL form can be well represented by a "refining sequence of partitions".

Definition 2.6 (refining sequence of partitions [15]). Let $\lambda=\lambda_{1} \ldots \lambda_{p}, \mu=\mu_{1} \ldots \mu_{q}$ be partitions of a natural number $m$ :

$$
\lambda_{1}+\cdots+\lambda_{p}=\mu_{1}+\cdots+\mu_{q}=m
$$

Here we assume that $\lambda_{i}$ 's and $\mu_{i}$ 's are not necessarily arranged in ascending or descending order.

If there exists a disjoint decomposition $\{1,2, \ldots, p\}=I_{1} \amalg \cdots \amalg I_{q}$ of the index set of $\lambda$ such that $\mu_{k}=\sum_{j \in I_{k}} \lambda_{j}$ holds, then we call $\lambda$ a refinement of $\mu$.

Let $\left[p_{0}, \ldots, p_{r}\right]$ be an $(r+1)$-tuple of partitions of $m$. When $p_{i+1}$ is a refinement of $p_{i}$ for all $i(i=0, \ldots, r-1)$, we call $\left[p_{0}, \ldots, p_{r}\right]$ a refining sequence of partitions, or $R S P$ for short. 
We denote an RSP in the following way.

Example 2.7. We consider the following RSP

$[321,2121,111111]$

as an example.

First, write the rightmost partition:

111111.

Second, put the numbers that are grouped together in the central partition in parentheses:

$$
(11)(1)(11)(1) \text {. }
$$

Finally, put the numbers that are grouped together in the leftmost partition in parentheses:

$$
((11)(1))((11))((1))
$$

Thus we can denote the above RSP by $((11)(1))((11))((1))$.

\subsubsection{Spectral types of ramified irregular singularities}

In general, non-semisimple matrices may appear in a sequence of block diagonalizations. Such a case can be reduced to the above semisimple case by "shearing transformations". Here we point out that, usually, the non-semisimplicity implies the ramifiedness of a singular point.

A shearing transformation is a gauge transformation by a diagonal matrix, which is typically of the form

$$
S=\operatorname{diag}\left(1, z^{s}, \ldots, z^{(m-1) s}\right),
$$

where $s$ is a positive rational number. The aim of the shearing transformation is to make nonsemisimple coefficient matrices semisimple by repeating gauge transformations of the above kind $[8,32]$. Instead of describing shearing transformations and constructions of HTL forms in the ramified case in full generality, we demonstrate a construction of an HTL form using the linear system (3.5). A general method of constructing HTL forms can be found in [33] (see also [14]).

First we change the dependent and independent variables as $z=1 / x, Y=\operatorname{diag}(1,-1,1) Z$, we rewrite (3.5) as follows

$$
\begin{aligned}
& \frac{\mathrm{d} Z}{\mathrm{~d} z}=A(z) Z \\
& A(z)=\left(\frac{A_{0}}{z^{2}}+\frac{A_{1}}{z}+A_{2}\right)
\end{aligned}
$$

where

$$
\begin{aligned}
A_{0} & =\left(\begin{array}{lll}
0 & 1 & 0 \\
0 & 0 & 0 \\
0 & 0 & 0
\end{array}\right), \quad A_{1}=\left(\begin{array}{ccc}
p_{2} q_{2} & -p_{2} & p_{1} p_{2} \\
0 & p_{1} q_{1}-p_{2} q_{2}-\theta_{2}^{0} & 1 \\
-t & q_{1} & -p_{1} q_{1}-\theta_{1}^{0}
\end{array}\right), \\
A_{2} & =\left(\begin{array}{ccc}
0 & 0 & 0 \\
q_{2} & -1 & p_{1} \\
0 & 0 & 0
\end{array}\right) .
\end{aligned}
$$

We consider the singular point $z=0$. 
Now we perform shearing transformations. Let $S_{1}$ be the diagonal matrix $\operatorname{diag}\left(1, z^{1 / 3}, z^{2 / 3}\right)$. Then we have

$$
\begin{aligned}
A^{S_{1}}(z)= & \frac{1}{z^{5 / 3}}\left(\begin{array}{ccc}
0 & 1 & 0 \\
0 & 0 & 0 \\
-t & 0 & 0
\end{array}\right)+\frac{1}{z^{4 / 3}}\left(\begin{array}{ccc}
0 & 0 & 0 \\
0 & 0 & 0 \\
0 & q_{1} & 0
\end{array}\right) \\
& +\frac{1}{z}\left(\begin{array}{ccc}
p_{2} q_{2} & 0 \\
0 & p_{1} q_{1}-p_{2} q_{2}-\theta_{2}^{0}-1 / 3 & 0 \\
0 & 0 & -p_{1} q_{1}-\theta_{1}^{0}-2 / 3
\end{array}\right)+\cdots
\end{aligned}
$$

How to find the rational number $s$ of the shearing matrix is described in [33]. In this case, the dimension of the centralizer of the leading coefficient matrix of $A^{S_{1}}(z)$ is less than that of $A(z)$. In fact, let $G_{1}$ be the following matrix

$$
G_{1}=\left(\begin{array}{ccc}
0 & 1 & 0 \\
0 & 0 & 1 \\
-t & 0 & 0
\end{array}\right)
$$

Then we have the following Jordan canonical form of the leading matrix of $A^{S_{1}}(z)$ :

$$
G_{1}^{-1}\left(\begin{array}{ccc}
0 & 1 & 0 \\
0 & 0 & 0 \\
-t & 0 & 0
\end{array}\right) G_{1}=\left(\begin{array}{ccc}
0 & 1 & 0 \\
0 & 0 & 1 \\
0 & 0 & 0
\end{array}\right) .
$$

Next, let $S_{2}=\operatorname{diag}\left(1, z^{1 / 3}, z^{2 / 3}\right)$. Then we have

$$
\begin{aligned}
A^{S_{1} G_{1} S_{2}}(z)= & \frac{1}{z^{4 / 3}}\left(\begin{array}{ccc}
0 & 1 & 0 \\
0 & 0 & 1 \\
-t & 0 & 0
\end{array}\right) \\
& +\frac{1}{z}\left(\begin{array}{ccc}
-p_{1} q_{1}-\theta_{1}^{0}-\frac{2}{3} & 0 & 0 \\
0 & p_{2} q_{2}-\frac{1}{3} & 0 \\
0 & 0 & p_{1} q_{1}-p_{2} q_{2}-\theta_{2}^{0}-1
\end{array}\right)+\cdots .
\end{aligned}
$$

Note that the leading matrix of $A^{S_{1} G_{1} S_{2}}(z)$ is diagonalizable. Indeed, the following matrix

$$
G_{2}=\left(\begin{array}{ccc}
1 & 1 & 1 \\
-t^{1 / 3} & -\omega t^{1 / 3} & -\omega^{2} t^{1 / 3} \\
t^{2 / 3} & \omega^{2} t^{2 / 3} & \omega t^{2 / 3}
\end{array}\right)
$$

diagonalizes the leading matrix of $A^{S_{1} G_{1} S_{2}}(z)$ :

$$
\begin{aligned}
A^{S_{1} G_{1} S_{2} G_{2}}(z)= & \frac{1}{z^{4 / 3}}\left(\begin{array}{ccc}
-t^{1 / 3} & 0 & 0 \\
0 & -\omega t^{1 / 3} & 0 \\
0 & 0 & -\omega^{2} t^{1 / 3}
\end{array}\right) \\
& +\frac{1}{z}\left(\begin{array}{ccc}
\theta_{1}^{\infty} / 3-2 / 3 & * & * \\
* & \theta_{1}^{\infty} / 3-2 / 3 & * \\
* & * & \theta_{1}^{\infty} / 3-2 / 3
\end{array}\right)+\cdots .
\end{aligned}
$$

Since the leading matrix has three distinct eigenvalues, we can remove the off-diagonal entries by virtue of Theorem 2.5. That is, there exits a matrix $P=P(z)$ whose entries are formal Laurent series in $z^{1 / 3}$ such that $A^{S_{1} G_{1} S_{2} G_{2} P}$ is diagonal:

$$
A^{S_{1} G_{1} S_{2} G_{2} P}(z)=\frac{1}{z^{4 / 3}}\left(\begin{array}{ccc}
-t^{1 / 3} & 0 & 0 \\
0 & -\omega t^{1 / 3} & 0 \\
0 & 0 & -\omega^{2} t^{1 / 3}
\end{array}\right)
$$




$$
+\frac{1}{z}\left(\begin{array}{ccc}
\theta_{1}^{\infty} / 3-2 / 3 & 0 & 0 \\
0 & \theta_{1}^{\infty} / 3-2 / 3 & 0 \\
0 & 0 & \theta_{1}^{\infty} / 3-2 / 3
\end{array}\right)+\cdots
$$

Then, by virtue of Theorem 2.4, we can truncate $A^{S_{1} G_{1} S_{2} G_{2} P}(z)$ after the principal part by a certain diagonal gauge transformation. Furthermore, by the scalar gauge transformation by $z^{-2 / 3}$, we can cancel the term $\frac{-2 / 3}{z}$. In this way, we can obtain the HTL form of (3.5) at $x=\infty$

$$
\frac{1}{z^{4 / 3}}\left(\begin{array}{ccc}
-t^{1 / 3} & 0 & 0 \\
0 & -\omega t^{1 / 3} & 0 \\
0 & 0 & -\omega^{2} t^{1 / 3}
\end{array}\right)+\frac{1}{z}\left(\begin{array}{ccc}
\theta_{1}^{\infty} / 3 & 0 & 0 \\
0 & \theta_{1}^{\infty} / 3 & 0 \\
0 & 0 & \theta_{1}^{\infty} / 3
\end{array}\right) .
$$

Together with the HTL form at $x=0$ (this can be easily seen), we obtain the Riemann scheme of the system (3.5):

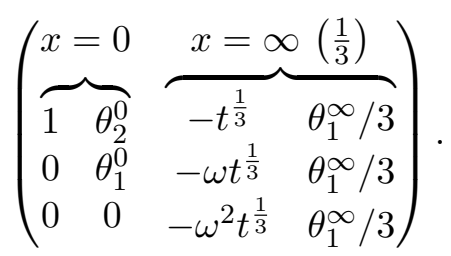

We briefly describe the feature of an HTL form (at a ramified irregular singularity) here. See [1] for a precise statement. Suppose the HTL form of $A(z) \in M_{m}(\mathbb{C}((z)))$ has

$$
\frac{T_{0}}{z^{\frac{b}{d}+1}}+\frac{T_{1}}{z^{\frac{b-1}{d}+1}}+\cdots+\frac{T_{b-1}}{z^{\frac{1}{d}+1}}+\frac{\Theta}{z}
$$

as its direct summand. Then the HTL form of $A(z)$ also has

$$
\left\{\frac{\zeta_{d}^{k b} T_{0}}{z^{\frac{b}{d}+1}}+\frac{\zeta_{d}^{k(b-1)} T_{1}}{z^{\frac{b-1}{d}+1}}+\cdots+\frac{\zeta_{d}^{k} T_{b-1}}{z^{\frac{1}{d}+1}}+\frac{\Theta}{z} \mid k=0, \ldots, d-1\right\}
$$

which is the orbit of (2.5) under the action $z^{\frac{1}{d}} \mapsto \zeta_{d} z^{\frac{1}{d}}\left(\zeta_{d}=e^{\frac{2 \pi i}{d}}\right)$ of a cyclic group, as its direct summands.

Let $S$ be the RSP corresponding to (2.5). We denote the collection of $S$ and its $d-1$ copies by $S_{d}$. Then an HTL form is generally represented as $S_{d_{1}}^{1} \ldots S_{d_{k}}^{k}$ where $S^{1}, \ldots, S^{k}$ are RSPs; we call this the spectral type of an HTL form. The spectral type at a singular point of a linear system is defined as the spectral type of the HTL form at the point. The tuple of the spectral types at all singular points is called the spectral type of the equation.

Example 2.8. The spectral types of

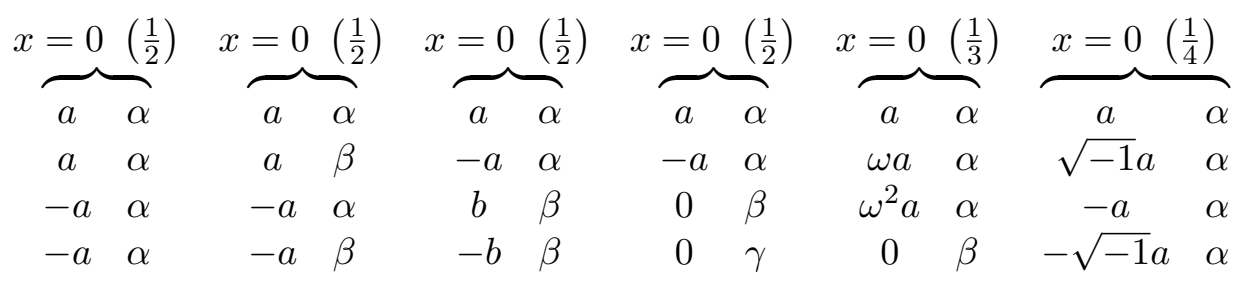

are $(2)_{2},(11)_{2},(1)_{2}(1)_{2},(1)_{2} 11,(1)_{3} 1$, and $(1)_{4}$ respectively. 


\subsection{Degeneration of HTL canonical forms}

Suppose a system of linear equations has some parameter, say $\varepsilon$. When we take the limit $\varepsilon \rightarrow 0$, usually with a gauge transformation by some matrix which depends on $\varepsilon$ and is independent of $x$, an HTL form of the linear system at some singular point may change. We call this situation a degeneration of an HTL form.

In the two dimensional case, the standard linear systems associated with classical Painlevé equations are $2 \times 2$, and degenerations of HTL forms are realized by the degenerations of the Jordan canonical forms of the coefficient matrices of the leading terms at irregular singular points. However, when the rank of a linear system is greater than two, a degeneration of an HTL form does not necessarily correspond to a degeneration of a Jordan canonical form. We can see such degenerations in the degeneration schemes of the Sasano system and the FujiSuzuki system. Here we take a sequence of degenerations $(11)(1),(11)(1) \rightarrow(1)_{2} 1,(11)(1) \rightarrow$ $(1)_{3},(11)(1)$ as an example.

Before going into the details, we look at the following simple example.

Example 2.9. Let $A(x)$ be a $2 \times 2$ matrix of the form

$$
A(x)=\frac{A_{0}}{x^{2}}+\frac{A_{1}}{x}+\cdots \in M_{2}(\mathbb{C}((x))),
$$

where

$$
A_{0}=\left(\begin{array}{ll}
0 & 1 \\
0 & 0
\end{array}\right), \quad A_{k}=\left(a_{i j}^{(k)}\right) \in M_{2}(\mathbb{C})
$$

Applying the gauge transformation by $S=\operatorname{diag}\left(1, x^{1 / 2}\right)$ to $(2.6)$, we have

$$
A^{S}(x)=\frac{1}{x^{3 / 2}}\left(\begin{array}{cc}
0 & 1 \\
a_{21}^{(1)} & 0
\end{array}\right)+\frac{1}{x}\left(\begin{array}{cc}
a_{11}^{(1)} & 0 \\
0 & a_{22}^{(1)}-\frac{1}{2}
\end{array}\right)+\cdots .
$$

When $a_{21}^{(1)} \neq 0$, the leading coefficient of $A^{S}(x)$ is a diagonalizable matrix with eigenvalues $\pm \sqrt{a_{21}^{(1)}}$. This means that the singular point $x=0$ of the system of linear differential equations corresponding to (2.6) is an irregular singular point of Poincaré rank $1 / 2$. We can see that the HTL form at $x=0$ is

$$
\overbrace{\sqrt{a_{21}^{(1)}} \operatorname{tr}\left(A_{1}\right)}^{x=0\left(\frac{1}{2}\right)}
$$

by diagonalizing the leading matrix. When $a_{21}^{(1)}=0$, using $\tilde{S}=\operatorname{diag}(1, x)$ instead of the above $S$, we have the different HTL form (we omit the details). This example implies that if the leading matrix is a Jordan canonical form whose $(1,2)$-entry is non-zero, then whether the $(2,1)$-entry of the subsequent matrix is zero or not is meaningful.

Now we consider the degeneration $(11)(1),(11)(1) \rightarrow(1)_{2} 1,(11)(1)$. The linear system of the spectral type (11)(1),(11)(1) is given by (A.1). Note that the leading matrix $A_{0}$ at the irregular singular point $x=\infty$ is diagonalizable. The degeneration of the HTL form at $x=\infty$ is caused by the degeneration of the Jordan canonical form of $A_{0}$ :

$$
\left(\begin{array}{ccc}
-t & & \\
& 0 & \\
& & 0
\end{array}\right) \rightarrow\left(\begin{array}{ccc}
0 & 1 & \\
& 0 & \\
& & 0
\end{array}\right), \quad t \rightarrow 0 .
$$


In fact, changing the variables of (A.1) as in Appendix A.2, we have the following new coefficient matrices

$$
\begin{aligned}
& \tilde{A}_{0}:=G^{-1} P U A_{0} U^{-1} P^{-1} G=\left(\begin{array}{ccc}
0 & \tilde{t} & 0 \\
0 & 0 & 0 \\
0 & 0 & 0
\end{array}\right)+O(\varepsilon), \\
& \tilde{A}_{1}:=G^{-1} \hat{A}_{1} G=\left(\begin{array}{ccc}
\left(\tilde{p}_{1}+\tilde{p}_{2}\right) \tilde{q}_{1} & \tilde{q}_{1} & \tilde{p}_{2} \tilde{q}_{1} \\
1 & -\tilde{p}_{1} \tilde{q}_{1}-\tilde{p}_{2} \tilde{q}_{2}+\theta_{1}^{0} & 1 \\
\tilde{p}_{2}\left(\tilde{q}_{2}-\tilde{q}_{1}\right)+\theta_{2}^{0}+\tilde{\theta}_{2}^{\infty} & \tilde{q}_{2}-\tilde{q}_{1} & \tilde{p}_{2}\left(\tilde{q}_{2}-\tilde{q}_{1}\right)+\theta_{2}^{0}
\end{array}\right)+O(\varepsilon), \\
& \tilde{A}_{2}:=G^{-1} \hat{A}_{2} G=\left(\begin{array}{ccc}
0 & 0 & 0 \\
\tilde{p}_{1}+\tilde{p}_{2} & 1 & \tilde{p}_{2} \\
0 & 0 & 0
\end{array}\right),
\end{aligned}
$$

where

$$
G=\left(\begin{array}{ccc}
0 & 1 & 0 \\
-1 / q_{2} & 0 & -1 / q_{2} \\
\varepsilon q_{1} & 0 & 0
\end{array}\right)
$$

Here $\tilde{A}_{0}$ is diagonalizable provided that $\varepsilon$ is not equal to 0 , and it degenerates to a nilpotent matrix when $\varepsilon$ tends to 0 . We note that the $(2,1)$-entry of $\lim _{\varepsilon \rightarrow 0} \tilde{A}_{1}$ is not equal to 0 , and thus we have the linear system (3.4) of the spectral type (1) $)_{2} 1$, (11)(1) by $\varepsilon \rightarrow 0$.

Remark 2.10. It is easy to obtain the HTL form at $x=\infty$, which corresponds to $(1)_{2} 1$, of (3.4). In the same manner as Example 2.9, the shearing at $x=\infty$ can be done by the matrix $S=\operatorname{diag}\left(1, x^{-1 / 2}, 1\right)$.

On the other hand, the degeneration of a HTL form $(1)_{2} 1 \rightarrow(1)_{3}$ does not correspond to the degeneration of a Jordan canonical form. Let us see the degeneration $(1)_{2} 1,(11)(1) \rightarrow$ $(1)_{3},(11)(1)$. In the course of the degeneration, the Jordan canonical form of the leading matrix stays unchanged. Instead, the $(2,1)$-entry of the subsequent matrix goes to zero. In fact, changing the variables of (3.4) as in Appendix A.2, we have

$$
\begin{aligned}
G^{-1} A_{0} G= & \left(\begin{array}{ccc}
0 & 1 & 0 \\
0 & 0 & 0 \\
0 & 0 & 0
\end{array}\right), \\
G^{-1} A_{1} G= & \left(\begin{array}{ccc}
-\tilde{p}_{2} \tilde{q}_{2} & -\tilde{p}_{2} & -\tilde{p}_{1} \tilde{p}_{2} \\
0 & -\tilde{p}_{1} \tilde{q}_{1}+\tilde{p}_{2} \tilde{q}_{2}+\tilde{\theta}_{2}^{0} & 1 \\
\tilde{t} & \tilde{q}_{1} & \tilde{p}_{1} \tilde{q}_{1}+\tilde{\theta}_{1}^{0}
\end{array}\right) \\
& +\varepsilon\left(\begin{array}{ccc}
-\tilde{t} \tilde{p}_{1} \tilde{p}_{2} & 0 & 0 \\
\tilde{t} & 0 & 0 \\
\tilde{t}\left(\tilde{p}_{1} \tilde{q}_{1}+\tilde{\theta}_{1}^{0}\right) & \tilde{t} \tilde{p}_{2} & \tilde{t}_{1} \tilde{p}_{2}
\end{array}\right)+O\left(\varepsilon^{2}\right),
\end{aligned}
$$

where $G=\operatorname{diag}(t, 1,1)$ (we have omitted the expression of $G^{-1} A_{2} G$ ). The limit $\varepsilon \rightarrow 0$ causes the degeneration of the HTL form $(1)_{2} 1 \rightarrow(1)_{3}$. In this way, we obtain the system (3.5). The construction of the HTL form at $x=\infty$ of (3.5) has been given in Section 2.3.2.

We determine the possibility of degeneration as follows. For example, $(1)_{2} 1$ is a direct sum of two direct summands (see the Riemann scheme of $(1)_{2} 1,(11)(1)$ )

$$
\frac{1}{z^{3 / 2}}\left(\begin{array}{cc}
\sqrt{t} & 0 \\
0 & -\sqrt{t}
\end{array}\right)+\frac{1}{z}\left(\begin{array}{cc}
\theta_{1}^{\infty} / 2 & 0 \\
0 & \theta_{1}^{\infty} / 2
\end{array}\right)
$$


and $\theta_{2}^{\infty} / z$. From this, we expect that it is possible to take a limit $t \rightarrow 0$ and indeed this corresponds to the degeneration $(1)_{2} 1 \rightarrow(1)_{3}$. On the other hand, $(1)_{3}$ itself consists of a single Galois orbit, see (2.4). Thus we conclude that (1) $)_{3}$ does not admit degeneration.

Let us make a remark on degeneration of HTL forms. We do not consider the degeneration of the HTL form (2)(1) since the degeneration of (2)(1) does not preserve the number of accessory parameters.

Remark 2.11. Accessory parameters of a linear system are free parameters remaining in the linear system when the Riemann scheme is fixed. The number of accessory parameters of a linear system coincides with the dimension of the phase space of the corresponding Painlevétype equation.

To see this, for example, suppose that the HTL form $(2)(1)$ of the $(2)(1), 111,111$-system degenerates. Then the degenerated linear system has the following form:

$$
\frac{\mathrm{d} Y}{\mathrm{~d} x}=\left\{\left(\begin{array}{ccc}
0 & 1 & 0 \\
0 & 0 & 0 \\
0 & 0 & 0
\end{array}\right)+\frac{A_{0}}{x}+\frac{A_{1}}{x-1}\right\} Y, \quad A_{*} \sim \operatorname{diag}\left(0, \theta_{1}^{*}, \theta_{2}^{*}\right), \quad *=0,1 .
$$

By a direct calculation, we find that the number of accessory parameters of the above system is six. In fact, there is a Fuchsian equation with spectral type 21,111,111,111, which has six accessory parameters. The system (2.7) turns out to be the degenerated system of the $21,111,111,111$-system. The same argument applies to the degeneration of $((11))((1))$ of the $((11))((1)), 111$-system.

\section{Lax pairs of degenerate FS and Garnier systems}

The Garnier systems and the Fuji-Suzuki systems are non-linear differential equations, which are regarded as generalizations of the Painlevé equations.

The Garnier system in $N$ variables was derived as the isomonodromic deformation equation of a second order Fuchsian equation with $N+3$ singular points [5]. The Fuji-Suzuki systems were originally derived from the Drinfeld-Sokolov hierarchy by similarity reductions [3].

In Section 3.1, we present the Riemann schemes, Lax pairs, and corresponding Hamiltonians of degenerate Garnier systems. In Section 3.2, we present similar data of degenerate Fuji-Suzuki systems.

\section{1 degenerate Garnier systems}

The Garnier systems were originally derived from a second order single Fuchsian equation with $N+3$ singular points. However, unlike the original study by Garnier, we adopt first order systems concerning linear equations. In [28], the Garnier system in $N$ variables was derived from the first order $2 \times 2$ system of the form

$$
\frac{\mathrm{d} Y}{\mathrm{~d} x}=\left(\frac{A_{0}}{x}+\frac{A_{1}}{x-1}+\sum_{j=1}^{N} \frac{A_{t_{j}}}{x-t_{j}}\right) Y .
$$

When $N$ equals 2, the Painlevé-type equation corresponding to (3.1) has a four-dimensional phase space. In [15], confluences from this linear system were considered.

The degeneration of the Garnier system in two variables was considered by Kimura [17]. He treated mainly the confluence of singular points of associated linear equations, and he obtained the degenerated Garnier systems with the singularity pattern $2+1+1+1,3+1+1,2+2+1$, 
$3+2,4+1,5$, and 9/2. Kawamuko [16] further considered the degeneration of HTL canonical forms and obtained eight degenerate Garnier systems.

In this subsection, we give the Riemann schemes, Lax pairs, and Hamiltonians for degenerate Garnier systems associated with ramified linear equations. Although all the Hamiltonians in this subsection are equivalent to those in $[17,16]$, we recalculated them. The following is the list of Hamiltonians for the degenerate Garnier systems associated with ramified linear equations:

$$
\begin{aligned}
& t_{1} H_{\mathrm{Gar}, t_{1}}^{\frac{3}{2}+1+1+1}\left(\begin{array}{c}
\alpha, \beta \\
\gamma
\end{array} ;_{t_{2}}^{t_{1}} ; \begin{array}{c}
q_{1}, p_{1} \\
q_{2}, p_{2}
\end{array}\right)=t_{1} H_{\mathrm{III}\left(D_{6}\right)}\left(-\alpha, \gamma-\alpha ; t_{1} ; q_{1}, p_{1}\right)+q_{1}\left(q_{1} p_{1}-\alpha\right) p_{2} \\
& +\frac{t_{1}}{t_{1}-t_{2}}\left(p_{1}\left(q_{1}-q_{2}\right)-\alpha\right)\left(p_{2}\left(q_{2}-q_{1}\right)-\beta\right), \\
& t_{2} H_{\text {Gar }, t_{2}}^{\frac{3}{2}+1+1+1}\left(\begin{array}{c}
\alpha, \beta \\
\gamma
\end{array} ; \begin{array}{c}
t_{1} \\
t_{2}
\end{array} ; \begin{array}{l}
q_{1}, p_{1} \\
q_{2}, p_{2}
\end{array}\right)=t_{2} H_{\mathrm{III}\left(D_{6}\right)}\left(-\beta, \gamma-\beta ; t_{2} ; q_{2}, p_{2}\right)+q_{2}\left(q_{2} p_{2}-\beta\right) p_{1} \\
& +\frac{t_{2}}{t_{2}-t_{1}}\left(p_{1}\left(q_{1}-q_{2}\right)-\alpha\right)\left(p_{2}\left(q_{2}-q_{1}\right)-\beta\right), \\
& H_{\mathrm{Gar}, t_{1}}^{\frac{5}{2}+1+1}\left(\alpha, \beta ; \begin{array}{c}
t_{1} \\
t_{2}
\end{array} ; \begin{array}{l}
q_{1}, p_{1} \\
q_{2}, p_{2}
\end{array}\right)=H_{\mathrm{II}}\left(-\alpha ; t_{1} ; q_{1}, p_{1}\right)+p_{1} p_{2} \\
& +\frac{1}{t_{1}-t_{2}}\left(p_{1}\left(q_{1}-q_{2}\right)-\alpha\right)\left(p_{2}\left(q_{2}-q_{1}\right)-\beta\right), \\
& H_{\mathrm{Gar}, t_{2}}^{\frac{5}{2}+1+1}\left(\alpha, \beta ; \begin{array}{c}
t_{1} \\
t_{2}
\end{array} \begin{array}{l}
q_{1}, p_{1} \\
q_{2}, p_{2}
\end{array}\right)=H_{\mathrm{II}}\left(-\beta ; t_{2} ; q_{2}, p_{2}\right)+p_{1} p_{2} \\
& +\frac{1}{t_{2}-t_{1}}\left(p_{1}\left(q_{1}-q_{2}\right)-\alpha\right)\left(p_{2}\left(q_{2}-q_{1}\right)-\beta\right), \\
& t_{1} H_{\mathrm{Gar}, t_{1}}^{2+\frac{3}{2}+1}\left(\alpha, \beta ; \begin{array}{l}
t_{1} \\
t_{2}
\end{array} ; \begin{array}{c}
q_{1}, p_{1} \\
q_{2}, p_{2}
\end{array}\right)=t_{1} H_{\mathrm{III}\left(D_{6}\right)}\left(-\alpha,-\beta-\alpha ; t_{1} ; q_{1}, p_{1}\right)+t_{2} p_{1} p_{2} \\
& -p_{2} q_{2}\left(2 p_{1} q_{1}-\alpha\right)+\frac{t_{2}}{t_{1}} p_{1} q_{1}-\frac{q_{1} q_{2}}{t_{1}}\left(p_{1} q_{1}-\alpha\right), \\
& t_{2} H_{\mathrm{Gar}, t_{2}}^{2+\frac{3}{2}+1}\left(\alpha, \beta ; \begin{array}{l}
t_{1} \\
t_{2}
\end{array} ; \begin{array}{l}
q_{1}, p_{1} \\
q_{2}, p_{2}
\end{array}\right)=t_{2} H_{\mathrm{III}\left(D_{7}\right)}\left(\beta+1 ; t_{2} ; q_{2}, p_{2}\right)-t_{2} p_{1} p_{2} \\
& -\frac{t_{2}}{t_{1}} p_{1} q_{1}+\frac{q_{1} q_{2}}{t_{1}}\left(p_{1} q_{1}-\alpha\right) \\
& H_{\text {Gar }, t_{1}}^{\frac{7}{2}+1}\left(\alpha ; \begin{array}{c}
t_{1} \\
t_{2}
\end{array} ; \begin{array}{l}
q_{1}, p_{1} \\
q_{2}, p_{2}
\end{array}\right)=H_{\mathrm{I}}\left(t_{1} ; q_{1}, p_{1}\right)+p_{2}\left(2 q_{1}-q_{2}^{2}-t_{2}\right)+\alpha q_{2}, \\
& H_{\text {Gar }, t_{2}}^{\frac{7}{2}+1}\left(\alpha ; \frac{t_{1}}{t_{2}} ; \begin{array}{l}
q_{1}, p_{1} \\
q_{2}, p_{2}
\end{array}\right)=p_{2}^{2}-t_{2} p_{2} q_{2}^{2}-t_{2}^{2} p_{2}+\alpha t_{2} q_{2} \\
& +2 p_{1} p_{2} q_{2}-q_{1} q_{2}\left(p_{2} q_{2}-\alpha\right)-p_{2} q_{1}\left(q_{1}-t_{2}\right)-t_{1} p_{2}-\alpha p_{1}, \\
& t_{1} H_{\mathrm{Gar}, t_{1}}^{3+\frac{3}{2}}\left(\alpha ; \begin{array}{l}
t_{1} \\
t_{2}
\end{array} ; \begin{array}{l}
q_{1}, p_{1} \\
q_{2}, p_{2}
\end{array}\right)=t_{1} H_{\mathrm{III}\left(D_{7}\right)}\left(\alpha ; t_{1} ; q_{1}, p_{1}\right)+p_{2} q_{2}\left(p_{2} q_{2}+\alpha\right)-q_{2} \\
& +q_{1}\left(p_{2} q_{1}+2 p_{1} p_{2} q_{2}-t_{2}-1\right) \\
& H_{\mathrm{Gar}, t_{2}}^{3+\frac{3}{2}}\left(\alpha ; \begin{array}{c}
t_{1} \\
t_{2}
\end{array} ; \begin{array}{l}
q_{1}, p_{1} \\
q_{2}, p_{2}
\end{array}\right)=p_{2} q_{1}\left(p_{1} q_{1}+2 p_{2} q_{2}+\alpha-1\right)+p_{1} q_{2}-q_{1}+t_{1} p_{2}-t_{2} p_{2} q_{2}, \\
& H_{\text {Gar }, t_{1}}^{\frac{5}{2}+2}\left(\alpha ; \begin{array}{c}
t_{1} \\
t_{2}
\end{array} ; \begin{array}{c}
q_{1}, p_{1} \\
q_{2}, p_{2}
\end{array}\right)=H_{\mathrm{II}}\left(\alpha ; t_{1} ; q_{1}, p_{1}\right)-2 p_{2} q_{2} q_{1}-t_{2} p_{2}-q_{2}, \\
& t_{2} H_{\text {Gar }, t_{2}}^{\frac{5}{2}+2}\left(\alpha ; \begin{array}{c}
t_{1} \\
t_{2}
\end{array} ; \begin{array}{l}
q_{1}, p_{1} \\
q_{2}, p_{2}
\end{array}\right)=p_{2}^{2} q_{2}^{2}+\alpha p_{2} q_{2}+t_{2} p_{2}\left(p_{1}-q_{1}^{2}-t_{1}\right)-p_{1} q_{2}-t_{2} q_{1},
\end{aligned}
$$

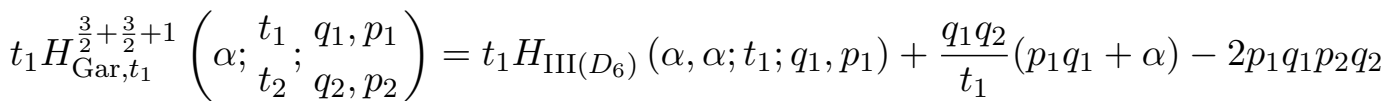




$$
\begin{aligned}
& -\alpha p_{2} q_{2}-\frac{t_{2} p_{1}}{q_{2}} \\
& t_{2} H_{\text {Gar }, t_{2}}^{\frac{3}{2}+\frac{3}{2}+1}\left(\alpha ; \begin{array}{l}
t_{1} \\
t_{2}
\end{array} ; \begin{array}{l}
q_{1}, p_{1} \\
q_{2}, p_{2}
\end{array}\right)=t_{2} H_{\mathrm{III}\left(D_{8}\right)}\left(t_{2} ; q_{2}, p_{2}\right)-\frac{q_{1} q_{2}}{t_{1}}\left(p_{1} q_{1}+\alpha\right)+\frac{t_{2} p_{1}}{q_{2}}, \\
& H_{\text {Gar }, t_{1}}^{\frac{9}{2}}\left(\begin{array}{c}
t_{1} \\
t_{2}
\end{array} ; \begin{array}{l}
q_{1}, p_{1} \\
q_{2}, p_{2}
\end{array}\right)=-p_{1}{ }^{3} p_{2}+{p_{1}}^{2}{q_{2}}^{2}+t_{1} p_{1}{ }^{3}-2 p_{1} q_{1} q_{2}-2 p_{1} p_{2}{ }^{2}+p_{2} q_{2}{ }^{2} \\
& +t_{1} p_{1} p_{2}+q_{1}^{2}-t_{1} q_{2}^{2}+t_{1}^{2} p_{1}+t_{2} p_{2}, \\
& H_{\text {Gar }, t_{2}}^{\frac{9}{2}}\left(\begin{array}{c}
t_{1} \\
t_{2}
\end{array} ; \begin{array}{l}
q_{1}, p_{1} \\
q_{2}, p_{2}
\end{array}\right)=p_{1}^{4}+3 p_{1}^{2} p_{2}+p_{1} q_{2}^{2}-2 q_{1} q_{2}+p_{2}{ }^{2}-t_{2} p_{1}+t_{1} p_{2}, \\
& H_{\text {Gar }, t_{1}}^{\frac{5}{2}+\frac{3}{2}}\left(\begin{array}{c}
t_{1} \\
t_{2}
\end{array} ; \begin{array}{l}
q_{1}, p_{1} \\
q_{2}, p_{2}
\end{array}\right)=H_{\mathrm{II}}\left(0 ; t_{1} ; q_{1}, p_{1}\right)-2 p_{2} q_{2} q_{1}-q_{2}-\frac{t_{2}}{q_{2}}, \\
& t_{2} H_{\mathrm{Gar}, t_{2}}^{\frac{5}{2}+\frac{3}{2}}\left(\begin{array}{c}
t_{1} \\
t_{2}
\end{array} ; \begin{array}{l}
q_{1}, p_{1} \\
q_{2}, p_{2}
\end{array}\right)={p_{2}}^{2} q_{2}^{2}-p_{1} q_{2}+\frac{t_{2}}{q_{2}}\left(p_{1}-q_{1}^{2}-t_{1}\right) \text {. }
\end{aligned}
$$

\section{Singularity pattern $\frac{3}{2}+1+1+1$}

The Riemann scheme is given by

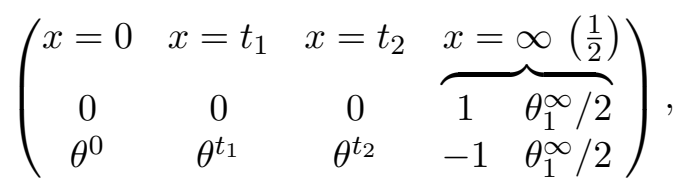

and the Fuchs-Hukuhara relation is written as $\theta^{0}+\theta^{t_{1}}+\theta^{t_{2}}+\theta_{1}^{\infty}=0$. The Lax pair is expressed as

$$
\begin{aligned}
& \frac{\partial Y}{\partial x}=\left(\frac{A_{0}}{x}+\frac{A_{t_{1}}}{x-t_{1}}+\frac{A_{t_{2}}}{x-t_{2}}+N\right) Y, \\
& \frac{\partial Y}{\partial t_{1}}=\left(N_{1}-\frac{A_{t_{1}}}{x-t_{1}}\right) Y, \quad \frac{\partial Y}{\partial t_{2}}=\left(N_{2}-\frac{A_{t_{2}}}{x-t_{2}}\right) Y .
\end{aligned}
$$

Here

$$
\begin{aligned}
& A_{0}=\left(\begin{array}{l}
0 \\
1
\end{array}\right)\left(1-p_{1}-p_{2} \quad \theta^{0}\right), \quad A_{t_{i}}=\left(\begin{array}{c}
q_{i} \\
1
\end{array}\right)\left(\begin{array}{ll}
p_{i} & \theta^{t_{i}}-p_{i} q_{i}
\end{array}\right), \\
& N=\left(\begin{array}{ll}
0 & 1 \\
0 & 0
\end{array}\right), \quad N_{i}=\frac{q_{i}\left(p_{i} q_{i}-\theta^{t_{i}}\right)}{t_{i}} N, \quad i=1,2 .
\end{aligned}
$$

The Hamiltonians are given by

$$
\begin{aligned}
t_{1} H_{\mathrm{Gar}, t_{1}}^{\frac{3}{2}+1+1+1}\left(\begin{array}{c}
\theta^{t_{1}}, \theta^{t_{2}}: t_{1}, q_{1}, p_{1} \\
-\theta^{0} ; t_{2} ; q_{2}, p_{2}
\end{array}\right)= & t_{1} H_{\mathrm{III}\left(D_{6}\right)}\left(-\theta^{t_{1}},-\theta^{0}-\theta^{t_{1}} ; t_{1} ; q_{1}, p_{1}\right)+q_{1}\left(q_{1} p_{1}-\theta^{t_{1}}\right) p_{2} \\
& +\frac{t_{1}}{t_{1}-t_{2}}\left(p_{1}\left(q_{1}-q_{2}\right)-\theta^{t_{1}}\right)\left(p_{2}\left(q_{2}-q_{1}\right)-\theta^{t_{2}}\right), \\
t_{2} H_{\mathrm{Gar}, t_{2}}^{\frac{3}{2}+1+1+1}\left(\begin{array}{c}
\theta^{t_{1}}, \theta^{t_{2}} ; t_{1} ; q_{1}, p_{1} \\
-\theta^{0} ; t_{2} ; q_{2}, p_{2}
\end{array}\right)= & t_{2} H_{\mathrm{III}\left(D_{6}\right)}\left(-\theta^{t_{2}},-\theta^{0}-\theta^{t_{2}} ; t_{2} ; q_{2}, p_{2}\right)+q_{2}\left(q_{2} p_{2}-\theta^{t_{2}}\right) p_{1} \\
& +\frac{t_{2}}{t_{2}-t_{1}}\left(p_{1}\left(q_{1}-q_{2}\right)-\theta^{t_{1}}\right)\left(p_{2}\left(q_{2}-q_{1}\right)-\theta^{t_{2}}\right) .
\end{aligned}
$$




\section{Singularity pattern $\frac{5}{2}+1+1$}

The Riemann scheme is given by

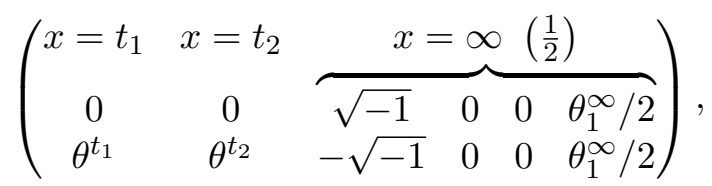

and the Fuchs-Hukuhara relation is written as $\theta^{t_{1}}+\theta^{t_{2}}+\theta_{1}^{\infty}=0$. The Lax pair is given as

$$
\begin{aligned}
& \frac{\partial Y}{\partial x}=\left(\frac{A_{t_{1}}}{x-t_{1}}+\frac{A_{t_{2}}}{x-t_{2}}+A_{\infty 1}+N x\right) Y, \\
& \frac{\partial Y}{\partial t_{1}}=\left(N_{1}-\frac{A_{t_{1}}}{x-t_{1}}\right) Y, \quad \frac{\partial Y}{\partial t_{2}}=\left(N_{2}-\frac{A_{t_{2}}}{x-t_{2}}\right) Y .
\end{aligned}
$$

Here

$$
\begin{aligned}
& A_{t_{i}}=\left(\begin{array}{c}
-q_{i} \\
1
\end{array}\right)\left(\begin{array}{ll}
-p_{i} & -p_{i} q_{i}+\theta^{t_{i}}
\end{array}\right), \quad N=\left(\begin{array}{ll}
0 & 1 \\
0 & 0
\end{array}\right), \\
& A_{\infty 1}=\left(\begin{array}{cc}
0 & -p_{1}-p_{2} \\
-1 & 0
\end{array}\right), \quad N_{i}=-p_{i} N, \quad i=1,2 .
\end{aligned}
$$

The Hamiltonians are written as

$$
\begin{aligned}
& H_{\mathrm{Gar}, t_{1}}^{\frac{5}{2}+1+1}\left(\theta^{t_{1}}, \theta^{t_{2}} ; \begin{array}{l}
t_{1} \\
t_{2}
\end{array} ; \begin{array}{l}
q_{1}, p_{1} \\
q_{2}, p_{2}
\end{array}\right) \\
& =H_{\mathrm{II}}\left(-\theta^{t_{1}} ; t_{1} ; q_{1}, p_{1}\right)+p_{1} p_{2}+\frac{1}{t_{1}-t_{2}}\left(p_{1}\left(q_{1}-q_{2}\right)-\theta^{t_{1}}\right)\left(p_{2}\left(q_{2}-q_{1}\right)-\theta^{t_{2}}\right), \\
& H_{\mathrm{Gar}, t_{1}}^{\frac{5}{2}+1+1}\left(\theta^{t_{1}}, \theta^{t_{2}} ;{ }_{t_{2}}^{t_{1}} ; \begin{array}{l}
q_{1}, p_{1} \\
q_{2}, p_{2}
\end{array}\right) \\
& =H_{\mathrm{II}}\left(-\theta^{t_{2}} ; t_{2} ; q_{2}, p_{2}\right)+p_{1} p_{2}+\frac{1}{t_{2}-t_{1}}\left(p_{1}\left(q_{1}-q_{2}\right)-\theta^{t_{1}}\right)\left(p_{2}\left(q_{2}-q_{1}\right)-\theta^{t_{2}}\right) \text {. }
\end{aligned}
$$

\section{Singularity pattern $2+\frac{3}{2}+1$}

The Riemann scheme is given by

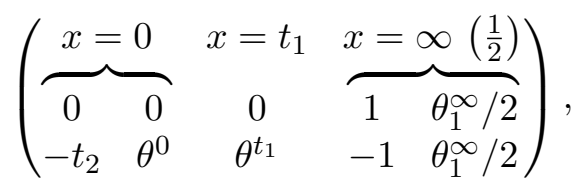

and the Fuchs-Hukuhara relation is written as $\theta^{0}+\theta^{t_{1}}+\theta_{1}^{\infty}=0$. The Lax pair is expressed as

$$
\begin{aligned}
& \frac{\partial Y}{\partial x}=\left(\frac{A_{0}^{(1)}}{x^{2}}+\frac{A_{0}^{(0)}}{x}+\frac{A_{t_{1}}}{x-t_{1}}+N\right) Y, \\
& \frac{\partial Y}{\partial t_{1}}=\left(N_{1}-\frac{A_{t_{1}}}{x-t_{1}}\right) Y, \quad \frac{\partial Y}{\partial t_{2}}=\left(N_{2}-\frac{\frac{1}{t_{2}} A_{0}^{(1)}}{x}\right) Y .
\end{aligned}
$$

Here

$$
A_{0}^{(1)}=\left(\begin{array}{cc}
0 & 0 \\
q_{2} & -t_{2}
\end{array}\right), \quad A_{0}^{(0)}=\left(\begin{array}{cc}
-p_{2} q_{2} & t_{2} p_{2} \\
1-p_{1} & p_{2} q_{2}+\theta^{0}
\end{array}\right), \quad N=\left(\begin{array}{ll}
0 & 1 \\
0 & 0
\end{array}\right),
$$




$$
A_{t_{1}}=\left(\begin{array}{c}
q_{1} \\
1
\end{array}\right)\left(\begin{array}{ll}
p_{1} & \theta^{t_{1}}-p_{1} q_{1}
\end{array}\right), \quad N_{1}=\frac{q_{1}\left(p_{1} q_{1}-\theta^{t_{1}}\right)}{t_{1}} N, \quad N_{2}=-p_{2} N
$$

The Hamiltonians are given by

$$
\begin{aligned}
& t_{1} H_{\mathrm{Gar}, t_{1}}^{2+\frac{3}{2}+1}\left(\theta^{t_{1}}, \theta^{0} ; \begin{array}{c}
t_{1} \\
t_{2}
\end{array} ; \begin{array}{c}
q_{1}, p_{1} \\
q_{2}, p_{2}
\end{array}\right)=t_{1} H_{\mathrm{III}\left(D_{6}\right)}\left(-\theta^{t_{1}},-\theta^{0}-\theta^{t_{1}} ; t_{1} ; q_{1}, p_{1}\right)+t_{2} p_{1} p_{2} \\
& -p_{2} q_{2}\left(2 p_{1} q_{1}-\theta^{t_{1}}\right)+\frac{t_{2}}{t_{1}} p_{1} q_{1}-\frac{q_{1} q_{2}}{t_{1}}\left(p_{1} q_{1}-\theta^{t_{1}}\right), \\
& t_{2} H_{\mathrm{Gar}, t_{2}}^{2+\frac{3}{2}+1}\left(\theta^{t_{1}}, \theta^{0} ; \begin{array}{l}
t_{1} \\
t_{2}
\end{array} ; \begin{array}{l}
q_{1}, p_{1} \\
q_{2}, p_{2}
\end{array}\right) \\
& =t_{2} H_{\mathrm{III}\left(D_{7}\right)}\left(\theta^{0}+1 ; t_{2} ; q_{2}, p_{2}\right)-t_{2} p_{1} p_{2}-\frac{t_{2}}{t_{1}} p_{1} q_{1}+\frac{q_{1} q_{2}}{t_{1}}\left(p_{1} q_{1}-\theta^{t_{1}}\right) .
\end{aligned}
$$

\section{Singularity pattern $\frac{7}{2}+1$}

The Riemann scheme is given by

$$
\left(\begin{array}{ccccccc}
x=0 & \multicolumn{8}{c}{x=\infty\left(\frac{1}{2}\right)} \\
0 & \overbrace{1}^{1} & 0 & -\frac{3 t_{2}}{2} & 0 & \frac{t_{1}}{2}+\frac{3 t_{2}{ }^{2}}{8} & \theta_{1}^{\infty} / 2 \\
\theta^{0} & -1 & 0 & \frac{3 t_{2}}{2} & 0 & -\frac{t_{1}}{2}-\frac{3 t_{2}{ }^{2}}{8} & \theta_{1}^{\infty} / 2
\end{array}\right),
$$

and the Fuchs-Hukuhara relation is written as $\theta^{0}+\theta_{1}^{\infty}=0$. The Lax pair is expressed as

$$
\begin{aligned}
& \frac{\partial Y}{\partial x}=\left(\frac{A_{3}}{x}+A_{2}+A_{1} x+A_{0} x^{2}\right) Y, \\
& \frac{\partial Y}{\partial t_{1}}=\left(A_{0} x+B_{10}\right) Y, \quad \frac{\partial Y}{\partial t_{2}}=\left(-A_{0} x^{2}+B_{21} x+B_{20}\right) Y .
\end{aligned}
$$

Here

$$
\begin{aligned}
& A_{0}=\left(\begin{array}{ll}
0 & 1 \\
0 & 0
\end{array}\right), \quad A_{1}=\left(\begin{array}{cc}
0 & q_{1}-2 t_{2} \\
1 & 0
\end{array}\right), \\
& A_{2}=\left(\begin{array}{cc}
-p_{1} & -p_{2}+q_{1}^{2}-t_{2} q_{1}+t_{1}+t_{2}^{2} \\
-q_{1}-t_{2} & p_{1}
\end{array}\right), \quad A_{3}=\left(\begin{array}{c}
q_{2} \\
1
\end{array}\right)\left(\begin{array}{ll}
p_{2} & -p_{2} q_{2}+\theta^{0}
\end{array}\right), \\
& B_{10}=\left(\begin{array}{cc}
0 & 2 q_{1}-t_{2} \\
1 & 0
\end{array}\right), \quad B_{21}=\left(\begin{array}{cc}
0 & 2 t_{2}-q_{1} \\
-1 & 0
\end{array}\right), \\
& B_{20}=\left(\begin{array}{cc}
p_{1} & 2 p_{2}-q_{1}^{2}+t_{2} q_{1}-t_{1}-t_{2}^{2} \\
q_{1}+t_{2} & -p_{1}
\end{array}\right) .
\end{aligned}
$$

The Hamiltonians are given by

$$
\begin{aligned}
& H_{\text {Gar }, t_{1}}^{\frac{7}{2}+1}\left(\theta^{0} ; \begin{array}{l}
t_{1} \\
t_{2}
\end{array} \begin{array}{l}
q_{1}, p_{1} \\
q_{2}, p_{2}
\end{array}\right)=H_{\mathrm{I}}\left(t_{1} ; q_{1}, p_{1}\right)+p_{2}\left(2 q_{1}-q_{2}{ }^{2}-t_{2}\right)+\theta^{0} q_{2}, \\
& H_{\text {Gar }, t_{2}}^{\frac{7}{2}+1}\left(\theta^{0} ; \begin{array}{c}
t_{1} \\
t_{2} ;
\end{array} \begin{array}{l}
q_{1}, p_{1} \\
q_{2}, p_{2}
\end{array}\right)=p_{2}{ }^{2}-t_{2} p_{2} q_{2}^{2}-t_{2}^{2} p_{2}+\theta^{0} t_{2} q_{2}+2 p_{1} p_{2} q_{2}-q_{1} q_{2}\left(p_{2} q_{2}-\theta^{0}\right) \\
& \quad-p_{2} q_{1}\left(q_{1}-t_{2}\right)-t_{1} p_{2}-\theta^{0} p_{1} .
\end{aligned}
$$




\section{Singularity pattern $3+\frac{3}{2}$}

The Riemann scheme is given by

$$
\left(\begin{array}{ccccc}
\overbrace{\begin{array}{c}
\sqrt{t_{1}} \\
-\sqrt{t_{1}}
\end{array}}^{x=0} & 0 & \overbrace{}^{\left(\frac{1}{2}\right)} & -t_{2} & \theta_{2}^{\infty}
\end{array}\right),
$$

and the Fuchs-Hukuhara relation is written as $\theta_{1}^{\infty}+\theta_{2}^{\infty}=0$. The Lax pair is expressed as

$$
\frac{\partial Y}{\partial x}=\left(\frac{A_{3}}{x^{2}}+\frac{A_{2}}{x}+A_{1}+A_{0} x\right) Y, \quad \frac{\partial Y}{\partial t_{1}}=-\frac{A_{3}}{t_{1} x} Y, \quad \frac{\partial Y}{\partial t_{2}}=\left(E_{2} x+B_{1}\right) Y .
$$

Here

$$
\begin{aligned}
& A_{\xi}=\left(\begin{array}{ll}
1 & \\
& u
\end{array}\right)^{-1} \hat{A}_{\xi}\left(\begin{array}{ll}
1 & \\
& u
\end{array}\right), \quad E_{2}=\operatorname{diag}(0,1), \\
& \hat{A}_{3}=t_{1}\left(\begin{array}{c}
1 \\
p_{2}
\end{array}\right)\left(\begin{array}{cc}
-p_{2} & 1
\end{array}\right), \quad \hat{A}_{0}=-E_{2}, \quad \hat{A}_{1}=\left(\begin{array}{cc}
0 & q_{1} \\
p_{2}\left(p_{2} q_{1}-t_{2}\right)+p_{1} & t_{2}
\end{array}\right), \\
& \hat{A}_{2}=\left(\begin{array}{cc}
-p_{2} q_{1}\left(p_{2} q_{1}-t_{2}\right)-p_{1} q_{1}-\theta_{1}^{\infty} & q_{1}\left(p_{2} q_{1}-t_{2}\right)-q_{2} \\
\left(\hat{A}_{2}\right)_{21} & p_{2} q_{1}\left(p_{2} q_{1}-t_{2}\right)+p_{1} q_{1}+\theta_{1}^{\infty}
\end{array}\right), \\
& B_{1}=\left(\begin{array}{cc}
0 & \left(-A_{1}\right)_{12} \\
\left(-A_{1}\right)_{21} & 0
\end{array}\right),
\end{aligned}
$$

where

$$
\left(\hat{A}_{2}\right)_{21}=-p_{2}^{2} q_{1}\left(p_{2} q_{1}-t_{2}\right)-p_{2}\left(2 p_{1} q_{1}+p_{2} q_{2}+2 \theta_{1}^{\infty}\right)+1 .
$$

The Hamiltonians are given by

$$
\begin{aligned}
& t_{1} H_{\mathrm{Gar}, t_{1}}^{3+\frac{3}{2}}\left(2 \theta_{1}^{\infty} ; \begin{array}{c}
t_{1} ; \\
t_{2}
\end{array} ; \begin{array}{c}
q_{1}, p_{1} \\
q_{2}, p_{2}
\end{array}\right)=t_{1} H_{\mathrm{III}\left(D_{7}\right)}\left(2 \theta_{1}^{\infty} ; t_{1} ; q_{1}, p_{1}\right)+p_{2} q_{2}\left(p_{2} q_{2}+2 \theta_{1}^{\infty}\right)-q_{2} \\
& \quad+q_{1}\left(p_{2} q_{1}+2 p_{1} p_{2} q_{2}-t_{2}-1\right) \\
& H_{\mathrm{Gar}, t_{2}}^{3+\frac{3}{2}}\left(2 \theta_{1}^{\infty} ; \begin{array}{c}
t_{1} \\
t_{2} ;
\end{array} \begin{array}{l}
q_{1}, p_{1} \\
q_{2}, p_{2}
\end{array}\right)=p_{2} q_{1}\left(p_{1} q_{1}+2 p_{2} q_{2}+2 \theta_{1}^{\infty}-1\right)+p_{1} q_{2}-q_{1}+t_{1} p_{2}-t_{2} p_{2} q_{2} .
\end{aligned}
$$

The gauge parameter $u$ satisfies

$$
\frac{1}{u} \frac{\partial u}{\partial t_{1}}=-\frac{2}{t_{1}}\left(p_{1} q_{1}+p_{2} q_{2}+\theta_{1}^{\infty}\right), \quad \frac{1}{u} \frac{\partial u}{\partial t_{2}}=-2 p_{2} q_{1} .
$$

Singularity pattern $\frac{5}{2}+2$

The Riemann scheme is given by

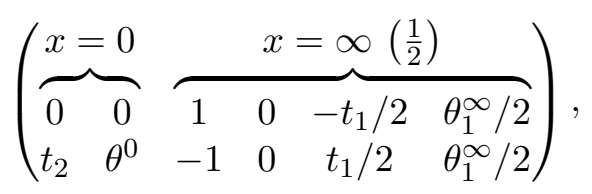

and the Fuchs-Hukuhara relation is written as $\theta^{0}+\theta_{1}^{\infty}=0$. The Lax pair is expressed as

$$
\frac{\partial Y}{\partial x}=\left(\frac{A_{3}}{x^{2}}+\frac{A_{2}}{x}+A_{1}+A_{0} x\right) Y
$$




$$
\frac{\partial Y}{\partial t_{1}}=\left(x B_{11}+B_{10}\right) Y, \quad \frac{\partial Y}{\partial t_{2}}=\left(-\frac{A_{3}}{t_{2} x}+B_{20}\right) Y
$$

where

$$
\begin{aligned}
& A_{0}=\left(\begin{array}{ll}
0 & 1 \\
0 & 0
\end{array}\right), \quad A_{1}=\left(\begin{array}{cc}
q_{1} & p_{1}-q_{1}^{2}-t_{1} \\
1 & -q_{1}
\end{array}\right), \\
& A_{2}=\left(\begin{array}{cc}
p_{2} q_{2} & q_{2} \\
-p_{1} & -p_{2} q_{2}-\theta_{1}^{\infty}
\end{array}\right), \quad A_{3}=\left(\begin{array}{cc}
0 & 0 \\
t_{2} p_{2} & t_{2}
\end{array}\right), \\
& B_{11}=\left(\begin{array}{cc}
0 & -1 \\
0 & 0
\end{array}\right), \quad B_{10}=\left(\begin{array}{cc}
-q_{1} & 0 \\
-1 & q_{1}
\end{array}\right), \quad B_{20}=\left(\begin{array}{cc}
0 & -q_{2} / t_{2} \\
0 & 0
\end{array}\right) .
\end{aligned}
$$

The Hamiltonians are given by

$$
\begin{aligned}
& H_{\text {Gar }, t_{1}}^{\frac{5}{2}+2}\left(\theta_{1}^{\infty} ; \begin{array}{c}
t_{1} ; \\
t_{2}
\end{array} ; \begin{array}{l}
q_{1}, p_{1} \\
q_{2}, p_{2}
\end{array}\right)=H_{\mathrm{II}}\left(\theta_{1}^{\infty} ; t_{1} ; q_{1}, p_{1}\right)-2 p_{2} q_{2} q_{1}-t_{2} p_{2}-q_{2}, \\
& t_{2} H_{\text {Gar }, t_{2}}^{\frac{5}{2}+2}\left(\theta_{1}^{\infty} ; \begin{array}{c}
t_{1} \\
t_{2} ;
\end{array} \begin{array}{l}
q_{1}, p_{1} \\
q_{2}, p_{2}
\end{array}\right)=p_{2}{ }^{2} q_{2}{ }^{2}+\theta_{1}^{\infty} p_{2} q_{2}+t_{2} p_{2}\left(p_{1}-q_{1}^{2}-t_{1}\right)-p_{1} q_{2}-t_{2} q_{1} .
\end{aligned}
$$

Singularity pattern $\frac{3}{2}+\frac{3}{2}+1$

The Riemann scheme is given by

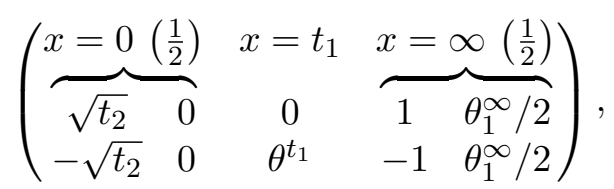

and the Fuchs-Hukuhara relation is written as $\theta^{t_{1}}+\theta_{1}^{\infty}=0$. The Lax pair is expressed as

$$
\begin{aligned}
& \frac{\partial Y}{\partial x}=\left(\frac{A_{0}^{(1)}}{x^{2}}+\frac{A_{0}^{(0)}}{x}+\frac{A_{t_{1}}}{x-t_{1}}+N\right) Y, \\
& \frac{\partial Y}{\partial t_{1}}=\left(N_{1}-\frac{A_{t_{1}}}{x-t_{1}}\right) Y, \quad \frac{\partial Y}{\partial t_{2}}=\left(N_{2}-\frac{\frac{1}{t_{2}} A_{0}^{(1)}}{x}\right) Y .
\end{aligned}
$$

Here

$$
\begin{aligned}
& A_{0}^{(1)}=\left(\begin{array}{cc}
0 & 0 \\
-q_{2} & 0
\end{array}\right), \quad A_{0}^{(0)}=\left(\begin{array}{cc}
-p_{2} q_{2} & -t_{2} / q_{2} \\
1-p_{1} & p_{2} q_{2}
\end{array}\right), \quad N=\left(\begin{array}{cc}
0 & 1 \\
0 & 0
\end{array}\right), \\
& A_{t_{1}}=\left(\begin{array}{c}
q_{1} \\
1
\end{array}\right)\left(\begin{array}{ll}
p_{1} & \theta^{t_{1}}-p_{1} q_{1}
\end{array}\right), \quad N_{1}=\frac{q_{1}\left(p_{1} q_{1}-\theta^{t_{1}}\right)}{t_{1}} N, \quad N_{2}=\frac{1}{q_{2}} N .
\end{aligned}
$$

The Hamiltonians are given by

$$
\begin{aligned}
& t_{1} H_{\mathrm{Gar}, t_{1}}^{\frac{3}{2}+\frac{3}{2}+1}\left(\theta_{1}^{\infty} ; \begin{array}{c}
t_{1} ; \\
t_{2}
\end{array} \begin{array}{l}
q_{1}, p_{1} \\
q_{2}, p_{2}
\end{array}\right) \\
& \quad=t_{1} H_{\mathrm{III}\left(D_{6}\right)}\left(\theta_{1}^{\infty}, \theta_{1}^{\infty} ; t_{1} ; q_{1}, p_{1}\right)+\frac{q_{1} q_{2}}{t_{1}}\left(p_{1} q_{1}+\theta_{1}^{\infty}\right)-2 p_{1} q_{1} p_{2} q_{2}-\theta_{1}^{\infty} p_{2} q_{2}-\frac{t_{2} p_{1}}{q_{2}}, \\
& t_{2} H_{\mathrm{Gar}, t_{2}}^{\frac{3}{2}+\frac{3}{2}+1}\left(\theta_{1}^{\infty} ; \begin{array}{c}
t_{1} \\
t_{2} ;
\end{array} \begin{array}{l}
q_{1}, p_{1} \\
q_{2}, p_{2}
\end{array}\right)=t_{2} H_{\mathrm{III}\left(D_{8}\right)}\left(t_{2} ; q_{2}, p_{2}\right)-\frac{q_{1} q_{2}}{t_{1}}\left(p_{1} q_{1}+\theta_{1}^{\infty}\right)+\frac{t_{2} p_{1}}{q_{2}} .
\end{aligned}
$$




\section{Singularity pattern $\frac{9}{2}$}

The Riemann scheme is given by

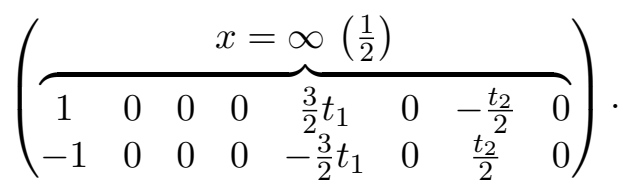

The Lax pair is expressed as

$$
\begin{aligned}
& \frac{\partial Y}{\partial x}=\left(A_{0} x^{3}+A_{1} x^{2}+A_{2} x+A_{3}\right) Y, \\
& \frac{\partial Y}{\partial t_{1}}=\left(A_{0} x^{2}+A_{1} x+B_{10}\right) Y, \quad \frac{\partial Y}{\partial t_{2}}=\left(-A_{0} x+B_{20}\right) Y,
\end{aligned}
$$

where

$$
\begin{aligned}
& A_{0}=\left(\begin{array}{ll}
0 & 1 \\
0 & 0
\end{array}\right), \quad A_{1}=\left(\begin{array}{cc}
0 & p_{1} \\
1 & 0
\end{array}\right), \quad A_{2}=\left(\begin{array}{cc}
q_{2} & p_{1}^{2}+p_{2}+2 t_{1} \\
-p_{1} & -q_{2}
\end{array}\right), \\
& A_{3}=\left(\begin{array}{cc}
q_{1}-p_{1} q_{2} & p_{1}^{3}+2 p_{1} p_{2}-q_{2}^{2}+t_{1} p_{1}-t_{2} \\
-p_{2}+t_{1} & -q_{1}+p_{1} q_{2}
\end{array}\right), \\
& B_{10}=\left(\begin{array}{cc}
q_{2} & p_{1}^{2}+2 p_{2}+t_{1} \\
-p_{1} & -q_{2}
\end{array}\right), \quad B_{20}=\left(\begin{array}{cc}
0 & -2 p_{1} \\
-1 & 0
\end{array}\right) .
\end{aligned}
$$

The Hamiltonians are given by

$$
\begin{aligned}
& H_{\text {Gar }, t_{1}}^{\frac{9}{2}}\left(\begin{array}{c}
t_{1} \\
t_{2}
\end{array} ; \begin{array}{l}
q_{1}, p_{1} \\
q_{2}, p_{2}
\end{array}\right)=-p_{1}{ }^{3} p_{2}+p_{1}{ }^{2} q_{2}{ }^{2}+t_{1} p_{1}{ }^{3}-2 p_{1} q_{1} q_{2}-2 p_{1} p_{2}{ }^{2}+p_{2} q_{2}{ }^{2} \\
& +t_{1} p_{1} p_{2}+q_{1}^{2}-t_{1} q_{2}^{2}+t_{1}^{2} p_{1}+t_{2} p_{2}, \\
& H_{\text {Gar }, t_{2}}^{\frac{9}{2}}\left(\begin{array}{c}
t_{1} \\
t_{2}
\end{array} ; \begin{array}{l}
q_{1}, p_{1} \\
q_{2}, p_{2}
\end{array}\right)=p_{1}^{4}+3 p_{1}^{2} p_{2}+p_{1} q_{2}^{2}-2 q_{1} q_{2}+p_{2}^{2}-t_{2} p_{1}+t_{1} p_{2} \text {. }
\end{aligned}
$$

\section{Singularity pattern $\frac{5}{2}+\frac{3}{2}$}

The Riemann scheme is given by

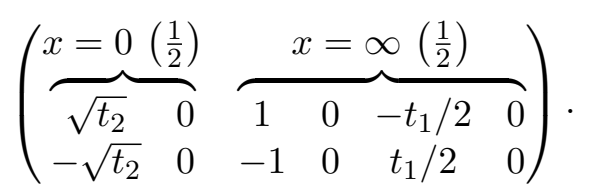

The Lax pair is expressed as

$$
\begin{aligned}
& \frac{\partial Y}{\partial x}=\left(\frac{A_{3}}{x^{2}}+\frac{A_{2}}{x}+A_{1}+A_{0} x\right) Y, \\
& \frac{\partial Y}{\partial t_{1}}=\left(x B_{11}+B_{10}\right) Y, \quad \frac{\partial Y}{\partial t_{2}}=\left(-\frac{A_{3}}{t_{2} x}+B_{20}\right) Y,
\end{aligned}
$$

where

$$
\begin{aligned}
& A_{0}=\left(\begin{array}{ll}
0 & 1 \\
0 & 0
\end{array}\right), \quad A_{1}=\left(\begin{array}{cc}
q_{1} & p_{1}-q_{1}^{2}-t_{1} \\
1 & -q_{1}
\end{array}\right), \\
& A_{2}=\left(\begin{array}{cc}
p_{2} q_{2} & q_{2} \\
-p_{1} & -p_{2} q_{2}
\end{array}\right), \quad A_{3}=\left(\begin{array}{cc}
0 & 0 \\
t_{2} / q_{2} & 0
\end{array}\right),
\end{aligned}
$$




$$
B_{11}=\left(\begin{array}{cc}
0 & -1 \\
0 & 0
\end{array}\right), \quad B_{10}=\left(\begin{array}{cc}
-q_{1} & 0 \\
-1 & q_{1}
\end{array}\right), \quad B_{20}=\left(\begin{array}{cc}
0 & -q_{2} / t_{2} \\
0 & 0
\end{array}\right)
$$

The Hamiltonians are given by

$$
\begin{aligned}
& H_{\mathrm{Gar}, t_{1}}^{\frac{5}{2}+\frac{3}{2}}\left(\begin{array}{c}
t_{1}, q_{1}, p_{1} \\
t_{2} ; \\
q_{2}, p_{2}
\end{array}\right)=H_{\mathrm{II}}\left(0 ; t_{1} ; q_{1}, p_{1}\right)-2 p_{2} q_{2} q_{1}-q_{2}-\frac{t_{2}}{q_{2}}, \\
& t_{2} H_{\mathrm{Gar}, t_{2}}^{\frac{5}{2}+\frac{3}{2}}\left(\begin{array}{l}
t_{1} \\
t_{2} ;
\end{array} ; \begin{array}{l}
q_{1}, p_{1} \\
q_{2}, p_{2}
\end{array}\right)={p_{2}}^{2} q_{2}^{2}-p_{1} q_{2}+\frac{t_{2}}{q_{2}}\left(p_{1}-q_{1}^{2}-t_{1}\right) .
\end{aligned}
$$

\subsection{Degenerate Fuji-Suzuki systems}

The Fuji-Suzuki systems were discovered by Fuji and Suzuki [3] in their study on similarity reductions of the Drinfeld-Sokolov hierarchies. A family of Painlevé-type equations which includes the Fuji-Suzuki system with $A_{5}$-symmetry was independently proposed by Tsuda [31].

Sakai [28] derived the Fuji-Suzuki system of type $A_{5}$ from the isomonodromic deformation of the following Fuchsian system:

$$
\frac{\mathrm{d} Y}{\mathrm{~d} x}=\left(\frac{A_{0}}{x}+\frac{A_{1}}{x-1}+\frac{A_{t}}{x-t}\right) Y
$$

where $A_{0}, A_{1}$, and $A_{t}$ are $3 \times 3$ matrices satisfying the following conditions

$$
A_{0} \sim \operatorname{diag}\left(0, \theta_{1}^{0}, \theta_{2}^{0}\right), \quad A_{1} \sim \operatorname{diag}\left(0,0, \theta^{1}\right), \quad A_{t} \sim \operatorname{diag}\left(0,0, \theta^{t}\right),
$$

and

$$
A_{\infty}:=-\left(A_{0}+A_{1}+A_{t}\right)=\operatorname{diag}\left(\theta_{1}^{\infty}, \theta_{2}^{\infty}, \theta_{3}^{\infty}\right) .
$$

Thus the spectral type of the Fuchsian system (3.2) is $21,21,111,111$. Taking the trace of (3.3), we have the Fuchs relation

$$
\theta_{1}^{0}+\theta_{2}^{0}+\theta^{1}+\theta^{t}+\theta_{1}^{\infty}+\theta_{2}^{\infty}+\theta_{3}^{\infty}=0 .
$$

The isomonodromic deformation equation of (3.2) is equivalent to the Hamiltonian system

$$
\frac{\mathrm{d} q_{i}}{\mathrm{~d} t}=\frac{\partial H_{\mathrm{FS}}^{A_{5}}}{\partial p_{i}}, \quad \frac{\mathrm{d} p_{i}}{\mathrm{~d} t}=-\frac{\partial H_{\mathrm{FS}}^{A_{5}}}{\partial q_{i}}, \quad i=1,2,
$$

where the Hamiltonian is given by

$$
\begin{aligned}
& H_{\mathrm{FS}}^{A_{5}}\left(\begin{array}{c}
\theta_{2}^{0}+\theta_{2}^{\infty}, \theta_{3}^{\infty}, \theta^{t} ; t ; \begin{array}{c}
q_{1}, p_{1} \\
\theta^{1}, \theta_{1}^{0}, \theta_{2}^{0}
\end{array} q_{2}, p_{2}
\end{array}\right)=H_{\mathrm{VI}}\left(\begin{array}{c}
\theta_{2}^{0}+\theta_{2}^{\infty}, \theta^{1}+\theta_{3}^{\infty} \\
\theta^{t}+\theta_{3}^{\infty}, \theta_{1}^{0}-\theta_{2}^{0}+1
\end{array} ; t ; q_{1}, p_{1}\right) \\
& +H_{\mathrm{VI}}\left(\begin{array}{c}
\theta_{3}^{\infty}, \theta_{2}^{0}+\theta^{1}+\theta_{2}^{\infty} \\
\theta_{2}^{0}+\theta^{t}+\theta_{2}^{\infty}, \theta_{1}^{0}-\theta_{2}^{0}-\theta_{2}^{\infty}+1
\end{array} ; t ; q_{2}, p_{2}\right) \\
& +\frac{1}{t(t-1)}\left(q_{1}-t\right)\left(q_{2}-1\right)\left\{\left(p_{1} q_{1}-\theta_{2}^{0}-\theta_{2}^{\infty}\right) p_{2}+p_{1}\left(p_{2} q_{2}-\theta_{3}^{\infty}\right)\right\} \text {. }
\end{aligned}
$$

The linear system of the spectral type $21,21,111,111$ has one deformation parameter. However, linear systems which are degenerated from the 21,21,111,111-system sometimes admit two-dimensional deformation, since a degeneration process does not necessarily preserve the number of deformation parameters. Such degenerations were pointed out in [15].

In the present paper, the following linear systems

$$
(1)_{2}(1), 21,21, \quad((1))(1)_{2}, 21, \quad(((1)))_{2} 1,21, \quad((1))_{3}, 21,21,
$$


$\left.(1)_{2}(1),(2)(1), \quad(((1)))(1)_{2}, \quad((1))_{3},(2)(1), \quad(((1)))\right)_{3}, 21$,

which are degenerated systems of the 21,21,111,111-system, also admit two-dimensional deformations and the Painlevé-type equations associated with these linear systems are degenerate Garnier systems.

The following are the Hamiltonians for the degenerate Fuji-Suzuki systems associated with ramified linear equations:

$$
\begin{aligned}
& t H_{\mathrm{Suz}}^{2+\frac{3}{2}}\left(\begin{array}{c}
\alpha, \beta \\
\gamma
\end{array} ; ; \begin{array}{l}
q_{1}, p_{1} \\
q_{2}, p_{2}
\end{array}\right) \\
& \quad=t H_{\mathrm{III}\left(D_{7}\right)}\left(\alpha ; t ; q_{1}, p_{1}\right)+t H_{\mathrm{III}\left(D_{7}\right)}\left(\beta ; t ; q_{2}, p_{2}\right)+p_{2} q_{1}\left(p_{1}\left(q_{1}+q_{2}\right)+\gamma\right)-q_{1}, \\
& t H_{\mathrm{KFS}}^{\frac{3}{2}+\frac{3}{2}}\left(\alpha, \beta ; t ; \begin{array}{l}
q_{1}, p_{1} \\
q_{2}, p_{2}
\end{array}\right) \\
& \quad=t H_{\mathrm{III}\left(D_{7}\right)}\left(\alpha ; t ; q_{1}, p_{1}\right)+t H_{\mathrm{III}\left(D_{7}\right)}\left(\beta ; t ; q_{2}, p_{2}\right)-p_{1} q_{1} p_{2} q_{2}-t\left(p_{1} p_{2}+p_{1}+p_{2}\right), \\
& t H_{\mathrm{KFS}}^{\frac{3}{2}+\frac{4}{3}}\left(\alpha ; t ; \begin{array}{l}
q_{1}, p_{1} \\
q_{2}, p_{2}
\end{array}\right) \\
& \quad=t H_{\mathrm{III}\left(D_{7}\right)}\left(\alpha ; t ; q_{1}, p_{1}\right)+t H_{\mathrm{III}\left(D_{7}\right)}\left(1-\alpha ; t ; q_{2}, p_{2}\right)-p_{1} q_{1} p_{2} q_{2}-t\left(\frac{p_{2}}{q_{1}}+p_{1}+p_{2}\right), \\
& t H_{\mathrm{KFS}}^{\frac{4}{3}+\frac{4}{3}}\left(t ; \begin{array}{c}
q_{1}, p_{1} \\
q_{2}, p_{2}
\end{array}\right) \\
& \quad=t H_{\mathrm{III}\left(D_{8}\right)}\left(t ; q_{1}, p_{1}\right)+t H_{\mathrm{III}\left(D_{8}\right)}\left(t ; q_{2}, p_{2}\right)-p_{1} q_{1} p_{2} q_{2}+\left(\frac{q_{1} q_{2}}{t}+q_{1}+q_{2}\right) .
\end{aligned}
$$

Here the Hamiltonian $H_{\mathrm{Suz}}^{2+\frac{3}{2}}$ can be transformed into the Hamiltonian ${ }_{0} \mathcal{H}_{2}$ in [30] via the following canonical transformation:

$$
q_{1} \rightarrow \frac{t}{q_{1}}, \quad p_{1} \rightarrow-\frac{q_{1}}{t}\left(p_{1} q_{1}+\gamma\right), \quad q_{2} \rightarrow t p_{2}, \quad p_{2} \rightarrow-\frac{q_{2}}{t} .
$$

Singularity pattern $2+1+1$

Spectral type $(1)_{2}(1), 21,21$. The Riemann scheme is given by

$$
\left(\begin{array}{ccccc}
x=0 & x=1 & \multicolumn{3}{c}{x=\infty\left(\frac{1}{2}\right)} \\
0 & 0 & \overbrace{0} \sqrt{t_{2}} & \theta_{1}^{\infty} / 2 \\
0 & 0 & 0 & -\sqrt{t_{2}} & \theta_{1}^{\infty} / 2 \\
\theta^{0} & \theta^{1} & -t_{1} & 0 & \theta_{2}^{\infty}
\end{array}\right),
$$

and the Fuchs-Hukuhara relation is written as $\theta^{0}+\theta^{1}+\theta_{1}^{\infty}+\theta_{2}^{\infty}=0$. The Lax pair is expressed as

$$
\frac{\partial Y}{\partial x}=\left(A_{\infty}+\frac{A_{0}}{x}+\frac{A_{1}}{x-1}\right) Y, \quad \frac{\partial Y}{\partial t_{1}}=\left(E_{3} x+B_{1}\right) Y, \quad \frac{\partial Y}{\partial t_{2}}=\left(\frac{1}{t_{2}} N x+B_{2}\right) Y .
$$

Here

$$
\begin{aligned}
& A_{\infty}=\left(\begin{array}{ccc}
0 & 1 & 0 \\
0 & 0 & 0 \\
0 & 0 & t_{1}
\end{array}\right), \quad A_{0}=\left(\begin{array}{c}
0 \\
1 \\
q_{1}
\end{array}\right)\left(\begin{array}{lll}
t_{2}\left(1-p_{2}\right) & a_{0} & \left.p_{1}\left(1-q_{1}\right)-\theta_{2}^{\infty}\right)
\end{array}\right. \\
& A_{1}=\left(\begin{array}{c}
q_{2} \\
t_{2} \\
t_{2}\left(q_{1}-1\right)
\end{array}\right)\left(\begin{array}{lll}
p_{2} & a_{1} & \left.\frac{1}{t_{2}}\left(p_{2} q_{2}+\theta_{2}^{\infty}\right)\right)
\end{array}\right.
\end{aligned}
$$




$$
\begin{aligned}
& E_{3}=\operatorname{diag}(0,0,1), \quad N=\left(\begin{array}{lll}
0 & 1 & 0 \\
0 & 0 & 0 \\
0 & 0 & 0
\end{array}\right), \\
& B_{1}=\frac{1}{t_{1}}\left(\begin{array}{ccc}
0 & -\frac{p_{1} q_{1}}{t_{1}}-\frac{q_{1} q_{2}}{t_{2}}\left(p_{1} q_{1}+\theta_{2}^{\infty}\right) & \frac{p_{1}}{t_{1}}+\frac{q_{2}}{t_{2}}\left(p_{1} q_{1}+\theta_{2}^{\infty}\right) \\
0 & 0 & p_{1} \\
t_{2}\left(q_{1}-p_{2}\right) & \left(B_{1}\right)_{32} & \left(B_{1}\right)_{33}
\end{array}\right) \text {, } \\
& B_{2}=\frac{1}{t_{2}}\left(\begin{array}{ccc}
p_{2} q_{2}+\theta_{1}^{\infty} & \frac{p_{1} q_{1}}{t_{1}} & -\frac{p_{1}}{t_{1}} \\
t_{2} & 1-p_{2} q_{2} & 0 \\
0 & \frac{t_{2}}{t_{1}}\left(p_{2}-q_{1}\right) & 1-q_{2}\left(q_{1}+p_{2}-1\right)
\end{array}\right) \text {, }
\end{aligned}
$$

where

$$
\begin{aligned}
& a_{0}=p_{1} q_{1}\left(q_{1}-1\right)+\theta_{2}^{\infty} q_{1}+\theta^{0}, \\
& a_{1}=\frac{1}{t_{2}}\left(\left(p_{1} q_{1}+\theta_{2}^{\infty}\right)\left(1-q_{1}\right)-p_{2} q_{2}+\theta^{1}\right), \\
& \left(B_{1}\right)_{32}=\left(q_{1}-1\right)\left(p_{1} q_{1}-p_{2} q_{2}\right)+\left(\theta_{2}^{\infty}-\theta_{1}^{\infty}\right) q_{1}+\frac{t_{2}}{t_{1}}\left(q_{1}-p_{2}\right)-\theta^{1}-\theta_{2}^{\infty}, \\
& \left(B_{1}\right)_{33}=\left(p_{1}+t_{1}\right)\left(q_{1}-1\right)+\left(p_{1}+q_{2}\right) q_{1}-p_{2} q_{2} .
\end{aligned}
$$

The Hamiltonians are given by

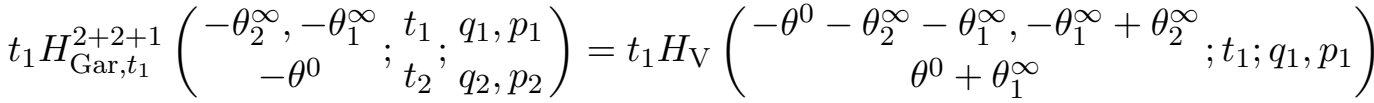

$$
\begin{aligned}
& +q_{1} q_{2}\left(p_{1} q_{1}+\theta_{2}^{\infty}\right)+p_{2} q_{2}\left(-\theta_{2}^{\infty}+p_{1}-2 p_{1} q_{1}\right)-\frac{t_{2}}{t_{1}} p_{1}\left(p_{2}-q_{1}\right), \\
& t_{2} H_{\mathrm{Gar}, t_{2}}^{2+2+1}\left(\begin{array}{c}
-\theta_{2}^{\infty},-\theta_{1}^{\infty} ; t_{1} ; \begin{array}{c}
q_{1}, p_{1} \\
-\theta^{0}
\end{array} t_{2} ; q_{2}, p_{2}
\end{array}\right)=t_{2} H_{\mathrm{III}\left(D_{6}\right)}\left(\theta^{0}+\theta_{1}^{\infty}+\theta_{2}^{\infty}, \theta_{1}^{\infty} ; t_{2} ; q_{2}, p_{2}\right) \\
& -\left(p_{1} q_{1}+\theta_{2}^{\infty}\right) q_{2}\left(q_{1}-1\right)+\frac{t_{2}}{t_{1}} p_{1}\left(p_{2}-q_{1}\right) .
\end{aligned}
$$

\section{Singularity pattern $3+1$}

Spectral type $((1))(1)_{2}, 21$. The Riemann scheme is given by

$$
\left(\begin{array}{cccccc}
x=0 & \multicolumn{4}{c}{x=\infty\left(\frac{1}{2}\right)} \\
0 & \overbrace{1} & 0 & t_{2} & 0 & \theta_{1}^{\infty} \\
0 & 0 & 0 & 0 & \sqrt{t_{1}} & \theta_{2}^{\infty} / 2 \\
\theta^{0} & 0 & 0 & 0 & -\sqrt{t_{1}} & \theta_{2}^{\infty} / 2
\end{array}\right),
$$

and the Fuchs-Hukuhara relation is written as $\theta^{0}+\theta_{1}^{\infty}+\theta_{2}^{\infty}=0$. The Lax pair is expressed as

$$
\frac{\partial Y}{\partial x}=\left(A_{0} x+A_{1}+\frac{A_{2}}{x}\right) Y, \quad \frac{\partial Y}{\partial t_{1}}=\left(B_{11} x+B_{10}\right) Y, \quad \frac{\partial Y}{\partial t_{2}}=\left(B_{21} x+B_{20}\right) Y,
$$

where

$$
\begin{aligned}
& A_{0}=\left(\begin{array}{ccc}
-1 & 0 & 0 \\
0 & 0 & 0 \\
0 & 0 & 0
\end{array}\right), \quad A_{1}=\left(\begin{array}{ccc}
-t_{2} & 1 & 0 \\
-p_{1} q_{1}+p_{2} q_{2}-\theta_{2}^{\infty} & 0 & 1 \\
q_{1} q_{2}+t_{1} & 0 & 0
\end{array}\right), \\
& A_{2}=\left(\begin{array}{c}
1 \\
p_{2} \\
q_{1}
\end{array}\right)\left(\begin{array}{ccc}
-p_{1} q_{1}+p_{2} q_{2}+\theta^{0} & -q_{2} & p_{1}
\end{array}\right),
\end{aligned}
$$




$$
\begin{array}{rlrl}
B_{11}= & \left(\begin{array}{ccc}
0 & 0 & 0 \\
0 & 0 & 1 / t_{1} \\
0 & 0 & 0
\end{array}\right), & B_{10}=\frac{1}{t_{1}}\left(\begin{array}{ccc}
0 & 0 & 1 \\
q_{1} q_{2}+t_{1} & 0 & q_{2}+t_{2} \\
0 & t_{1} & 2 p_{1} q_{1}+\theta_{2}^{\infty}+1
\end{array}\right), \\
B_{21}=\left(\begin{array}{ccc}
-1 & 0 & 0 \\
0 & 0 & 0 \\
0 & 0 & 0
\end{array}\right), & B_{20}=\left(\begin{array}{ccc}
0 & 1 & 0 \\
-p_{1} q_{1}+p_{2} q_{2}-\theta_{2}^{\infty} & q_{2}+t_{2} & 1-p_{1} \\
q_{1} q_{2}+t_{1} & 0 & q_{2}+t_{2}
\end{array}\right) .
\end{array}
$$

The Hamiltonians are given by

$$
\begin{aligned}
& H_{\mathrm{Gar}, t_{1}}^{3+2}\left(\theta_{2}^{\infty}, \theta^{0} ; \begin{array}{c}
t_{1} ; \\
t_{2} ;
\end{array} \begin{array}{l}
q_{1}, p_{1} \\
q_{2}, p_{2}
\end{array}\right) \\
& \quad=H_{\mathrm{III}\left(D_{6}\right)}\left(-\theta^{0}, \theta_{2}^{\infty}+1 ; t_{1} ; q_{1}, p_{1}\right)-p_{1}-\frac{q_{1} q_{2}}{t_{1}}\left(q_{2}-p_{2}+t_{2}\right)+p_{1} p_{2}-q_{2}, \\
& H_{\mathrm{Gar}, t_{2}}^{3+2}\left(\theta_{2}^{\infty}, \theta^{0} ; \begin{array}{c}
t_{1} \\
\left.t_{2} ; \begin{array}{l}
q_{1}, p_{1} \\
q_{2}, p_{2}
\end{array}\right)=H_{\mathrm{IV}}\left(\theta_{2}^{\infty}, \theta^{0} ; t_{2} ; q_{2}, p_{2}\right)-p_{1} q_{1}\left(p_{2}-2 q_{2}-t_{2}\right)-q_{1} q_{2}+t_{1} p_{1} .
\end{array}\right.
\end{aligned}
$$

\section{Singularity pattern $\frac{5}{3}+1+1$}

Spectral type $((1))_{3}, 21,21$. The Riemann scheme is given by

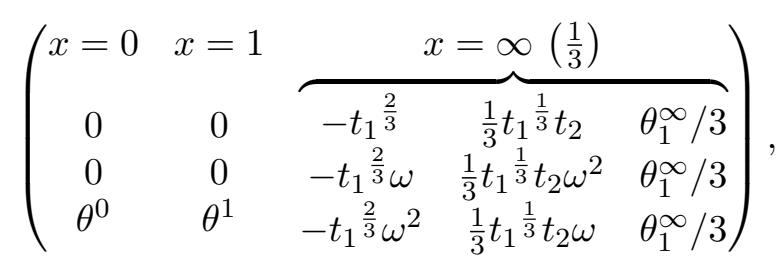

where $\omega=e^{2 \pi i / 3}$ is a cube root of unity. The Fuchs-Hukuhara relation is written as $\theta^{0}+\theta^{1}+\theta_{1}^{\infty}$ $=0$. The Lax pair is expressed as

$$
\frac{\partial Y}{\partial x}=\left(A_{\infty}+\frac{A_{0}}{x}+\frac{A_{1}}{x-1}\right) Y, \quad \frac{\partial Y}{\partial t_{1}}=\left(B_{11} x+B_{10}\right) Y, \quad \frac{\partial Y}{\partial t_{2}}=\left(B_{21} x+B_{20}\right) Y .
$$

Here

$$
\begin{aligned}
& A_{\infty}=\left(\begin{array}{lll}
0 & 1 & 0 \\
0 & 0 & 1 \\
0 & 0 & 0
\end{array}\right), \quad A_{0}=\left(\begin{array}{l}
0 \\
0 \\
1
\end{array}\right)\left(\begin{array}{lll}
-t_{1} q_{1} q_{2} & a_{0} & \theta^{0}
\end{array}\right),
\end{aligned}
$$

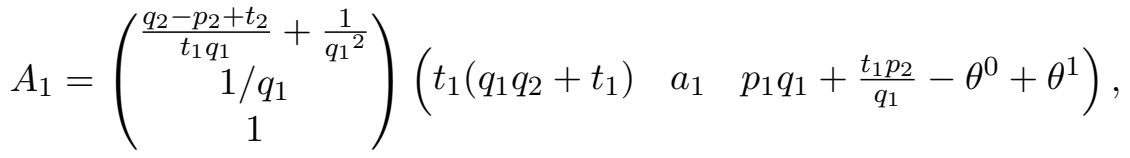

$$
\begin{aligned}
& B_{11}=\frac{1}{t_{1}}\left(\begin{array}{ccc}
0 & 1 & 0 \\
0 & 0 & 1 \\
0 & 0 & 0
\end{array}\right), \quad B_{21}=-\frac{1}{t_{1}}\left(\begin{array}{ccc}
0 & 0 & 1 \\
0 & 0 & 0 \\
0 & 0 & 0
\end{array}\right) \text {, } \\
& B_{10}=\frac{1}{t_{1}}\left(\begin{array}{ccc}
\left(B_{10}\right)_{11} & \left(B_{10}\right)_{12} & 0 \\
q_{2}+\frac{t_{1}}{q_{1}} & \left(B_{10}\right)_{22} & 0 \\
t_{1} & -q_{2}-t_{2}-\frac{t_{1}}{q_{1}} & \frac{1}{t_{1}}\left(p_{1} q_{1}-\theta^{0}+2\right)+\frac{p_{2}}{q_{1}}
\end{array}\right) \text {, } \\
& B_{20}=\left(\begin{array}{ccc}
-q_{2}-t_{2}-\frac{t_{1}}{q_{1}} & \left(B_{20}\right)_{12} & 0 \\
-t_{1} & 0 & 0 \\
0 & -t_{1} & q_{2}+\frac{t_{1}}{q_{1}}
\end{array}\right)
\end{aligned}
$$

where

$$
a_{0}=q_{1} q_{2}\left(q_{2}-p_{2}+t_{2}\right)+q_{1}\left(p_{1} q_{1}-\theta^{0}\right)+t_{1} q_{2},
$$




$$
\begin{aligned}
& a_{1}=q_{1} q_{2}\left(p_{2}-q_{2}-t_{2}\right)-q_{1}\left(p_{1} q_{1}-\theta^{0}\right)-t_{1}\left(2 q_{2}+\frac{t_{1}}{q_{1}}+t_{2}\right), \\
& \left(B_{10}\right)_{11}=\frac{1}{t_{1}}\left(\left(q_{2}+\frac{t_{1}}{q_{1}}\right)\left(q_{2}-p_{2}+t_{2}\right)+\theta_{1}^{\infty}\right)+\frac{q_{1} q_{2}+t_{1}}{q_{1}^{2}}, \\
& \left(B_{10}\right)_{12}=\frac{1}{t_{1}^{2}}\left(\left(p_{1} q_{1}-q_{2}\left(p_{2}-q_{2}-t_{2}\right)+\frac{t_{1}\left(3 q_{2}+t_{2}\right)}{q_{1}}-\theta^{0}\right)\left(p_{2}-q_{2}-t_{2}\right)-2 t_{1} p_{1}\right) \\
& +\frac{2 \theta^{0}-\theta^{1}}{t_{1} q_{1}}-\frac{3 q_{2}+2 t_{2}}{q_{1}^{2}}-\frac{t_{1}}{q_{1}^{3}} \\
& \left(B_{10}\right)_{22}=\frac{1}{t_{1}}\left(q_{2}\left(p_{2}-q_{2}-t_{2}\right)-p_{1} q_{1}-\theta^{1}+1\right)-\frac{2 q_{2}+t_{2}}{q_{1}}-\frac{t_{1}}{q_{1}^{2}}, \\
& \left(B_{20}\right)_{12}=\frac{1}{t_{1}}\left(\left(q_{2}+\frac{2 t_{1}}{q_{1}}\right)\left(p_{2}-q_{2}-t_{2}\right)+p_{1} q_{1}+\theta^{1}-1\right)+\frac{t_{2}}{q_{1}}-\frac{t_{1}}{q_{1}^{2}} .
\end{aligned}
$$

The Hamiltonians are given by

$$
\begin{aligned}
& H_{\mathrm{Gar}, t_{1}}^{3+2}\left(\theta^{1}-\theta^{0}, \theta^{0} ; \begin{array}{c}
t_{1} \\
t_{2}
\end{array} \begin{array}{c}
q_{1}, p_{1} \\
q_{2}, p_{2}
\end{array}\right) \\
& \quad=H_{\mathrm{III}\left(D_{6}\right)}\left(-\theta^{0}, \theta^{1}-\theta^{0}+1 ; t_{1} ; q_{1}, p_{1}\right)-p_{1}-\frac{q_{1} q_{2}}{t_{1}}\left(q_{2}-p_{2}+t_{2}\right)+p_{1} p_{2}-q_{2}, \\
& H_{\mathrm{Gar}, t_{2}}^{3+2}\left(\theta^{1}-\theta^{0}, \theta^{0} ; \begin{array}{c}
t_{1} \\
t_{2} ; \\
q_{1}, p_{1} \\
q_{2}, p_{2}
\end{array}\right) \\
& \quad=H_{\mathrm{IV}}\left(\theta^{1}-\theta^{0}, \theta^{0} ; t_{2} ; q_{2}, p_{2}\right)-p_{1} q_{1}\left(p_{2}-2 q_{2}-t_{2}\right)-q_{1} q_{2}+t_{1} p_{1} .
\end{aligned}
$$

\section{Singularity pattern $\frac{5}{2}+1$}

Spectral type $(((1)))_{2} 1,21$. The Riemann scheme is given by

$$
\left(\begin{array}{ccccc}
x=0 & \multicolumn{4}{c}{x=\infty\left(\frac{1}{2}\right)} \\
0 & \overbrace{1} t_{2} & -t_{1} / 2 & \theta_{1}^{\infty} / 2 \\
0 & -1 & t_{2} & t_{1} / 2 & \theta_{1}^{\infty} / 2 \\
\theta^{0} & 0 & 0 & 0 & \theta_{2}^{\infty}
\end{array}\right),
$$

and the Fuchs-Hukuhara relation is written as $\theta^{0}+\theta_{1}^{\infty}+\theta_{2}^{\infty}=0$. The Lax pair is expressed as

$$
\frac{\partial Y}{\partial x}=\left(A_{0} x+A_{1}+\frac{A_{2}}{x}\right) Y, \quad \frac{\partial Y}{\partial t_{1}}=\left(B_{11} x+B_{10}\right) Y, \quad \frac{\partial Y}{\partial t_{2}}=\left(B_{21} x+B_{20}\right) Y .
$$

Here

$$
\begin{aligned}
& A_{0}=\left(\begin{array}{ccc}
0 & 1 & 0 \\
0 & 0 & 0 \\
0 & 0 & 0
\end{array}\right), \quad A_{1}=\left(\begin{array}{ccc}
q_{1}-t_{2} & p_{1}-q_{1}^{2}-t_{1} & -1 \\
1 & -q_{1}-t_{2} & 0 \\
0 & p_{2} q_{2}-\theta_{2}^{\infty} & 0
\end{array}\right) \\
& A_{2}=\left(\begin{array}{c}
0 \\
1 \\
-p_{2}
\end{array}\right)\left(\begin{array}{lll}
-p_{1} & p_{2} q_{2}+\theta^{0} & q_{2}
\end{array}\right) \text {, } \\
& B_{11}=\left(\begin{array}{ccc}
0 & -1 & 0 \\
0 & 0 & 0 \\
0 & 0 & 0
\end{array}\right), \quad B_{10}=\left(\begin{array}{ccc}
-q_{1} & p_{2} & 1 \\
-1 & q_{1} & 0 \\
0 & -p_{2} q_{2}+\theta_{2}^{\infty} & q_{2}-t_{2}
\end{array}\right) \text {, } \\
& B_{21}=\left(\begin{array}{ccc}
-1 & 0 & 0 \\
0 & -1 & 0 \\
0 & 0 & 0
\end{array}\right), \quad B_{20}=\left(\begin{array}{ccc}
0 & p_{2}\left(q_{1}-q_{2}+t_{2}\right) & q_{1}-q_{2}+t_{2} \\
0 & 0 & 1 \\
-p_{2} q_{2}+\theta_{2}^{\infty} & \left(B_{20}\right)_{32} & \left(B_{20}\right)_{33}
\end{array}\right) \text {, }
\end{aligned}
$$


where

$$
\left(B_{20}\right)_{32}=-p_{1} p_{2}+\left(p_{2} q_{2}-\theta_{2}^{\infty}\right)\left(q_{1}-t_{2}\right), \quad\left(B_{20}\right)_{33}=p_{1}-t_{1}-t_{2}^{2}-q_{2}\left(q_{1}-t_{2}\right) .
$$

The Hamiltonians are given by

$$
\begin{aligned}
& H_{\mathrm{Gar}, t_{1}}^{4+1}\left(\theta_{2}^{\infty}, \theta^{0} ; \begin{array}{c}
t_{1} \\
t_{2}
\end{array} ; \begin{array}{c}
q_{1}, p_{1} \\
q_{2}, p_{2}
\end{array}\right)=H_{\mathrm{II}}\left(-\theta^{0} ; t_{1} ; q_{1}, p_{1}\right)+p_{2} q_{2}\left(q_{1}-q_{2}+t_{2}\right)+p_{1} p_{2}+\theta_{2}^{\infty} q_{2},
\end{aligned}
$$

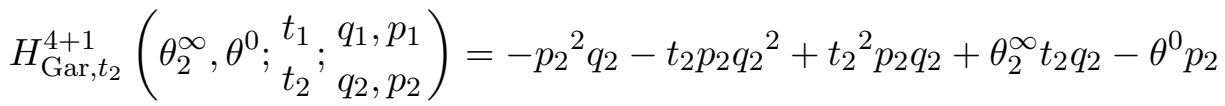

$$
\begin{aligned}
& +p_{1} p_{2}\left(q_{1}-2 q_{2}+t_{2}\right)+q_{1} q_{2}\left(p_{2} q_{2}-\theta_{2}^{\infty}\right)+\theta_{2}^{\infty} p_{1}+t_{1} p_{2} q_{2} \text {. }
\end{aligned}
$$

\section{Singularity pattern $2+2$}

Spectral type $(1)_{2}(1),(2)(1)$. The Riemann scheme is given by

$$
\left(\begin{array}{ccccc}
x=0 & \multicolumn{2}{c}{x=\infty\left(\frac{1}{2}\right)} \\
\overbrace{0}^{x} & 0 & 0 & \sqrt{-t_{2}} & \theta_{1}^{\infty} / 2 \\
0 & 0 & 0 & -\sqrt{-t_{2}} & \theta_{1}^{\infty} / 2 \\
1 & \theta^{0} & -t_{1} & 0 & \theta_{2}^{\infty}
\end{array}\right),
$$

and the Fuchs-Hukuhara relation is written as $\theta^{0}+\theta_{1}^{\infty}+\theta_{2}^{\infty}=0$. The Lax pair is expressed as

$$
\frac{\partial Y}{\partial x}=\left(\frac{A_{2}}{x^{2}}+\frac{A_{1}}{x}+A_{0}\right) Y, \quad \frac{\partial Y}{\partial t_{1}}=\left(E_{3} x+B_{1}\right) Y, \quad \frac{\partial Y}{\partial t_{2}}=\left(\frac{1}{t_{2}} N x+B_{2}\right) Y .
$$

Here

$$
\begin{aligned}
& A_{0}=\left(\begin{array}{ccc}
0 & 1 & 0 \\
0 & 0 & 0 \\
0 & 0 & t_{1}
\end{array}\right), \quad A_{2}=\left(\begin{array}{l}
0 \\
1 \\
1
\end{array}\right)\left(\begin{array}{lll}
q_{2} & 1-p_{1} & p_{1}
\end{array}\right) \\
& A_{1}=\left(\begin{array}{ccc}
-p_{2} q_{2} & \left(p_{1}-1\right) p_{2} & -p_{1} p_{2} \\
-t_{2} & p_{2} q_{2}-\theta_{1}^{\infty} & -p_{1} q_{1}-\theta_{2}^{\infty} \\
q_{1} q_{2}-t_{2} & \left(1-p_{1}\right) q_{1}+p_{2} q_{2}-\theta_{1}^{\infty} & -\theta_{2}^{\infty}
\end{array}\right), \\
& E_{3}=\operatorname{diag}\left(\begin{array}{lll}
0 & 0 & 1
\end{array}\right), \quad N=\left(\begin{array}{ccc}
0 & 1 & 0 \\
0 & 0 & 0 \\
0 & 0 & 0
\end{array}\right) \text {, } \\
& B_{1}=\frac{1}{t_{1}}\left(\begin{array}{ccc}
0 & p_{1}\left(p_{2}+\frac{q_{1}}{t_{1}}\right)+\frac{\theta_{2}^{\infty}}{t_{1}} & -p_{1}\left(p_{2}+\frac{q_{1}}{t_{1}}\right)-\frac{\theta_{2}^{\infty}}{t_{1}} \\
0 & 0 & -p_{1} q_{1}-\theta_{2}^{\infty} \\
q_{1} q_{2}-t_{2} & \left(B_{1}\right)_{32} & \left(B_{1}\right)_{33}
\end{array}\right) \text {, } \\
& B_{2}=\frac{1}{t_{1} t_{2}}\left(\begin{array}{ccc}
-t_{1}\left(p_{2} q_{2}-\theta_{1}^{\infty}\right) & -p_{1} q_{1}-\theta_{2}^{\infty} & p_{1} q_{1}+\theta_{2}^{\infty} \\
-t_{1} t_{2} & t_{1}\left(p_{2} q_{2}+1\right) & 0 \\
0 & -q_{1} q_{2}+t_{2} & q_{1} q_{2}+t_{1}\left(p_{2} q_{2}+1\right)-t_{2},
\end{array}\right)
\end{aligned}
$$

where

$$
\begin{aligned}
& \left(B_{1}\right)_{32}=q_{1}\left(-p_{1}+\frac{q_{2}}{t_{1}}+1\right)+\left(p_{2} q_{2}-\theta_{1}^{\infty}-\frac{t_{2}}{t_{1}}\right), \\
& \left(B_{1}\right)_{33}=-q_{2}\left(p_{2}+\frac{q_{1}}{t_{1}}\right)-q_{1}+\theta_{1}^{\infty}-\theta_{2}^{\infty}+\frac{t_{2}}{t_{1}} .
\end{aligned}
$$


The Hamiltonians are given by

$$
\begin{aligned}
& t_{1} H_{\mathrm{Gar}, t_{1}}^{2+\frac{3}{2}+1}\left(-\theta_{2}^{\infty},-\theta_{1}^{\infty} ;{ }_{t_{2}}^{t_{1}} ; \begin{array}{c}
q_{1}, p_{1} \\
q_{2}, p_{2}
\end{array}\right)=t_{1} H_{\mathrm{III}\left(D_{6}\right)}\left(\theta_{2}^{\infty}, \theta_{1}^{\infty}+\theta_{2}^{\infty} ; t_{1} ; q_{1}, p_{1}\right)+t_{2} p_{1} p_{2} \\
& \quad-p_{2} q_{2}\left(2 p_{1} q_{1}+\theta_{2}^{\infty}\right)+\frac{t_{2}}{t_{1}} p_{1} q_{1}-\frac{q_{1} q_{2}}{t_{1}}\left(p_{1} q_{1}+\theta_{2}^{\infty}\right), \\
& t_{2} H_{\mathrm{Gar}, t_{2}}^{2+\frac{3}{2}+1}\left(-\theta_{2}^{\infty},-\theta_{1}^{\infty} ;{ }_{t_{2}}^{t_{1}} ; \begin{array}{c}
q_{1}, p_{1} \\
q_{2}, p_{2}
\end{array}\right)=t_{2} H_{\mathrm{III}\left(D_{7}\right)}\left(-\theta_{1}^{\infty}+1 ; t_{2} ; q_{2}, p_{2}\right)-t_{2} p_{1} p_{2} \\
& \quad-\frac{t_{2}}{t_{1}} p_{1} q_{1}+\frac{q_{1} q_{2}}{t_{1}}\left(p_{1} q_{1}+\theta_{2}^{\infty}\right) .
\end{aligned}
$$

\section{Singularity pattern $2+\frac{5}{3}$}

Spectral type $((1))_{3},(2)(1)$. The Riemann scheme is given by

$$
\left(\begin{array}{ccccc}
\overbrace{0}^{x=0} & 0 & \overbrace{-1} & \frac{t_{2}}{3} & \theta_{1}^{\infty} / 3 \\
0 & 0 & -\omega & \frac{\omega^{2} t_{2}}{3} & \theta_{1}^{\infty} / 3 \\
t_{1} & \theta^{0} & -\omega^{2} & \frac{\omega t_{2}}{3} & \theta_{1}^{\infty} / 3
\end{array}\right),
$$

and the Fuchs-Hukuhara relation is written as $\theta^{0}+\theta_{1}^{\infty}=0$. The Lax pair is expressed as

$$
\frac{\partial Y}{\partial x}=\left(\frac{A_{2}}{x^{2}}+\frac{A_{1}}{x}+A_{0}\right) Y, \quad \frac{\partial Y}{\partial t_{1}}=\left(\frac{B_{11}}{x}+B_{10}\right) Y, \quad \frac{\partial Y}{\partial t_{2}}=\left(B_{21} x+B_{20}\right) Y,
$$

where

$$
\begin{aligned}
& A_{0}=N, \quad A_{2}=t_{1}\left(\begin{array}{l}
0 \\
0 \\
1
\end{array}\right)\left(\begin{array}{lll}
-p_{2} & p_{1} & 1
\end{array}\right), \quad N=\left(\begin{array}{lll}
0 & 1 & 0 \\
0 & 0 & 1 \\
0 & 0 & 0
\end{array}\right), \\
& A_{1}=\left(\begin{array}{ccc}
p_{2} q_{2} & -p_{1} q_{2} & -q_{2} \\
-p_{2} q_{1} & p_{1} q_{1} & q_{1} \\
1 & p_{2} q_{1}-t_{2} & -p_{1} q_{1}-p_{2} q_{2}-\theta_{1}^{\infty}
\end{array}\right), \\
& B_{11}=-\frac{1}{t_{1}} A_{2}, \quad B_{21}=-N^{2}, \\
& B_{10}=\frac{1}{t_{1}}\left(\begin{array}{ccc}
0 & -q_{1} & q_{2} \\
0 & 0 & -q_{1} \\
0 & 0 & 0
\end{array}\right), \quad B_{20}=\left(\begin{array}{ccc}
p_{2} q_{1}-t_{2} & -p_{1} q_{1}-2 p_{2} q_{2}-\theta_{1}^{\infty}+1 & 0 \\
-1 & 0 & 0 \\
0 & -1 & -p_{2} q_{1}
\end{array}\right) .
\end{aligned}
$$

The Hamiltonians are given by

$$
\begin{aligned}
& t_{1} H_{\mathrm{Gar}, t_{1}}^{3+\frac{3}{2}}\left(\theta_{1}^{\infty} ; \begin{array}{c}
t_{1} \\
t_{2} ;
\end{array} \begin{array}{l}
q_{1}, p_{1} \\
q_{2}, p_{2}
\end{array}\right)=t_{1} H_{\mathrm{III}\left(D_{7}\right)}\left(\theta_{1}^{\infty} ; t_{1} ; q_{1}, p_{1}\right)+p_{2} q_{2}\left(p_{2} q_{2}+\theta_{1}^{\infty}\right)-q_{2} \\
& \quad+q_{1}\left(p_{2} q_{1}+2 p_{1} p_{2} q_{2}-t_{2}-1\right), \\
& H_{\mathrm{Gar}, t_{2}}^{3+\frac{3}{2}}\left(\theta_{1}^{\infty} ; \begin{array}{c}
t_{1} \\
t_{2} ;
\end{array} \begin{array}{l}
q_{1}, p_{1} \\
q_{2}, p_{2}
\end{array}\right)=p_{2} q_{1}\left(p_{1} q_{1}+2 p_{2} q_{2}+\theta_{1}^{\infty}-1\right)+p_{1} q_{2}-q_{1}+t_{1} p_{2}-t_{2} p_{2} q_{2} .
\end{aligned}
$$




\section{Singularity pattern 4}

Spectral type $(((1)))(1)_{2}$. The Riemann scheme is given by

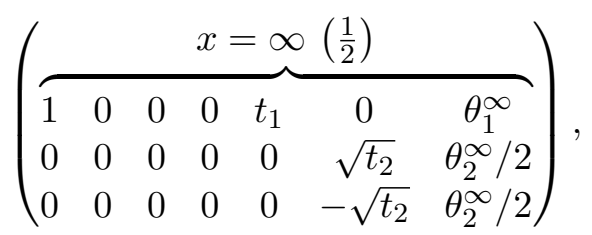

and the Fuchs-Hukuhara relation is written as $\theta_{1}^{\infty}+\theta_{2}^{\infty}=0$. The Lax pair is expressed as

$$
\frac{\partial Y}{\partial x}=\left(A_{0} x^{2}+A_{1} x+A_{2}\right) Y, \quad \frac{\partial Y}{\partial t_{1}}=\left(B_{11} x+B_{10}\right) Y, \quad \frac{\partial Y}{\partial t_{2}}=\left(B_{21} x+B_{20}\right) Y,
$$

where

$$
\begin{array}{rlrl}
A_{0}= & \left(\begin{array}{ccc}
-1 & 0 & 0 \\
0 & 0 & 0 \\
0 & 0 & 0
\end{array}\right), & A_{1}=\left(\begin{array}{ccc}
0 & 1 & 0 \\
p_{1} & 0 & 0 \\
p_{2} & 0 & 0
\end{array}\right), & A_{2}=\left(\begin{array}{ccc}
p_{1}-t_{1} & -q_{1} & -q_{2} \\
p_{1} q_{1}+p_{2} q_{2}+\theta_{1}^{\infty} & -p_{1} & t_{2} \\
p_{2} q_{1}+1 & -p_{2} & 0
\end{array}\right), \\
B_{11}=\left(\begin{array}{ccc}
-1 & 0 & 0 \\
0 & 0 & 0 \\
0 & 0 & 0
\end{array}\right), & B_{10}=\left(\begin{array}{ccc}
0 & 1 & 0 \\
p_{1} & q_{1} & q_{2} \\
p_{2} & 0 & q_{1}
\end{array}\right), \\
B_{21}=\left(\begin{array}{lll}
0 & 0 & 0 \\
0 & 0 & 1 \\
0 & 0 & 0
\end{array}\right), & B_{20}=\left(\begin{array}{ccc}
0 & 0 & 1 \\
p_{2} & 0 & q_{1} \\
0 & 1 / t_{2} & -\frac{1}{t_{2}}\left(2 p_{2} q_{2}+\theta_{1}^{\infty}\right.
\end{array}\right)
\end{array}
$$

The Hamiltonians are given by

$$
\begin{aligned}
& H_{\text {Gar }, t_{1}}^{\frac{5}{2}+2}\left(\theta_{1}^{\infty} ; \begin{array}{c}
t_{1} \\
t_{2}
\end{array} ; \begin{array}{l}
q_{1}, p_{1} \\
q_{2}, p_{2}
\end{array}\right)=H_{\mathrm{II}}\left(\theta_{1}^{\infty} ; t_{1} ; q_{1}, p_{1}\right)-2 p_{2} q_{2} q_{1}-t_{2} p_{2}-q_{2},
\end{aligned}
$$

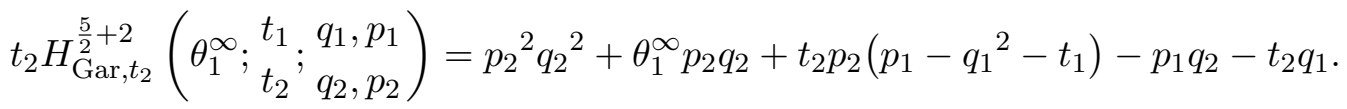

\section{Singularity pattern $\frac{7}{3}+1$}

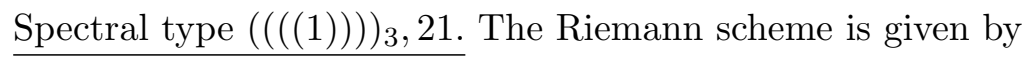

$$
\left(\begin{array}{cccccc}
x=0 & \multicolumn{6}{c}{x=\infty\left(\frac{1}{3}\right)} \\
0 & \overbrace{1} 0 & -\frac{2}{3} t_{2} & -t_{1} / 3 & \theta_{1}^{\infty} / 3 \\
0 & \omega & 0 & -\frac{2}{3} \omega^{2} t_{2} & -\omega t_{1} / 3 & \theta_{1}^{\infty} / 3 \\
\theta^{0} & \omega^{2} & 0 & -\frac{2}{3} \omega t_{2} & -\omega^{2} t_{1} / 3 & \theta_{1}^{\infty} / 3
\end{array}\right),
$$

and the Fuchs-Hukuhara relation is written as $\theta^{0}+\theta_{1}^{\infty}=0$. The Lax pair is expressed as

$$
\frac{\partial Y}{\partial x}=\left(A_{0} x+A_{1}+\frac{A_{2}}{x}\right) Y, \quad \frac{\partial Y}{\partial t_{1}}=\left(B_{11} x+B_{10}\right) Y, \quad \frac{\partial Y}{\partial t_{2}}=\left(B_{21} x+B_{20}\right) Y,
$$

where

$$
A_{0}=\left(\begin{array}{ccc}
0 & 1 & 0 \\
0 & 0 & 0 \\
0 & 0 & 0
\end{array}\right), \quad A_{1}=\left(\begin{array}{ccc}
0 & p_{1}-q_{1} q_{2}-t_{1} & -p_{2} \\
0 & 0 & -1 \\
1 & q_{2}-p_{2}+2 t_{2} & 0
\end{array}\right),
$$




$$
\begin{aligned}
& A_{2}=\left(\begin{array}{c}
0 \\
1 \\
q_{1}
\end{array}\right)\left(\begin{array}{lll}
q_{2} & -p_{1} q_{1}+\theta^{0} & p_{1}
\end{array}\right) \\
& B_{11}=\left(\begin{array}{ccc}
0 & -1 & 0 \\
0 & 0 & 0 \\
0 & 0 & 0
\end{array}\right), \quad B_{10}=\left(\begin{array}{ccc}
0 & -q_{1}\left(p_{2}+q_{2}\right) & p_{2}+q_{2} \\
0 & 0 & 1 \\
-1 & p_{2}-2\left(q_{2}+t_{2}\right) & 0
\end{array}\right) \text {, } \\
& B_{21}=\left(\begin{array}{ccc}
0 & 0 & -1 \\
0 & 0 & 0 \\
0 & 1 & 0
\end{array}\right), \quad B_{20}=\left(\begin{array}{ccc}
q_{2}-p_{2}+t_{2} & q_{1}\left(p_{1}-2 q_{1} q_{2}-t_{1}\right) & -p_{1}+2 q_{1} q_{2}+t_{1} \\
-1 & p_{2}-t_{2} & 0 \\
0 & 2 p_{1}-q_{1} q_{2}-t_{1} & -q_{2}-t_{2}
\end{array}\right) \text {. }
\end{aligned}
$$

The Hamiltonians are given by

$$
\begin{aligned}
& H_{\text {Gar }, t_{1}}^{5}\left(\theta^{0} ; \begin{array}{c}
t_{1} \\
t_{2}
\end{array} \begin{array}{l}
q_{1}, p_{1} \\
q_{2}, p_{2}
\end{array}\right)=-q_{1}\left(p_{1} q_{1}-\theta^{0}\right)+q_{2}\left(q_{1}\left(p_{2}+q_{2}\right)-2 p_{1}+t_{1}\right)+p_{1}\left(p_{2}-2 t_{2}\right), \\
& H_{\text {Gar }, t_{2}}^{5}\left(\theta^{0} ; \begin{array}{c}
t_{1} \\
t_{2} ;
\end{array} \begin{array}{l}
q_{1}, p_{1} \\
q_{2}, p_{2}
\end{array}\right)=H_{\mathrm{IV}}\left(-1, \theta^{0} ; 2 t_{2} ; q_{2}, p_{2}\right) \\
& \quad+q_{1} q_{2}\left(q_{1} q_{2}-2 p_{1}+t_{1}\right)+p_{1}\left(p_{1}-p_{2} q_{1}-t_{1}\right) .
\end{aligned}
$$

\section{Singularity pattern $\frac{3}{2}+1+1$}

Spectral type $(1)_{2} 1,21,111$. The Riemann scheme is given by

$$
\left(\begin{array}{cccc}
x=0 & x=1 & \overbrace{\sqrt{-t}}^{x=\infty} & \theta_{1}^{\infty} / 2 \\
0 & 0 & \left.\frac{1}{2}\right) \\
\theta_{1}^{0} & 0 & -\sqrt{-t} & \theta_{1}^{\infty} / 2 \\
\theta_{2}^{0} & \theta^{1} & 0 & \theta_{2}^{\infty}
\end{array}\right),
$$

and the Fuchs-Hukuhara relation is written as $\theta_{1}^{0}+\theta_{2}^{0}+\theta^{1}+\theta_{1}^{\infty}+\theta_{2}^{\infty}=0$.

The Lax pair is expressed as

$$
\frac{\partial Y}{\partial x}=\left(\frac{A_{0}}{x}+\frac{A_{1}}{x-1}+A_{\infty}\right) Y, \quad \frac{\partial Y}{\partial t}=\left(\frac{1}{t} A_{\infty} x+B_{0}\right) Y .
$$

Here

$$
\begin{aligned}
& A_{\xi}=P^{-1} \hat{A}_{\xi} P \quad(\xi=0,1), \quad P=\left(\begin{array}{ccc}
0 & 1 & 0 \\
1 & 0 & -t \\
\frac{1}{t}\left(p_{1} q_{1}-\theta_{2}^{\infty}\right) & 0 & 0
\end{array}\right), \\
& \hat{A}_{0}=\left(\begin{array}{ccc}
\theta_{1}^{0} & -p_{2} & q_{1} \\
0 & \theta_{2}^{0} & q_{1} q_{2}-t \\
0 & 0 & 0
\end{array}\right), \quad \hat{A}_{1}=\left(\begin{array}{c}
1 \\
q_{2} \\
p_{1}
\end{array}\right)\left(p_{1} q_{1}+\left(\begin{array}{lll}
\left.1-p_{2}\right) q_{2}+\theta^{1} & p_{2}-1 & -q_{1}
\end{array}\right),\right. \\
& A_{\infty}=\left(\begin{array}{lll}
0 & t & 0 \\
0 & 0 & 0 \\
0 & 0 & 0
\end{array}\right), \quad B_{0}=\frac{1}{t}\left(\begin{array}{ccc}
\left(A_{0}+A_{1}\right)_{11} & 0 & 0 \\
-1 & \left(A_{0}+A_{1}\right)_{22} & t \\
\left(A_{0}+A_{1}\right)_{31} & 0 & \left(p_{2}-1\right) q_{2}+\theta_{2}^{0}-1
\end{array}\right) .
\end{aligned}
$$

The Hamiltonian is given by

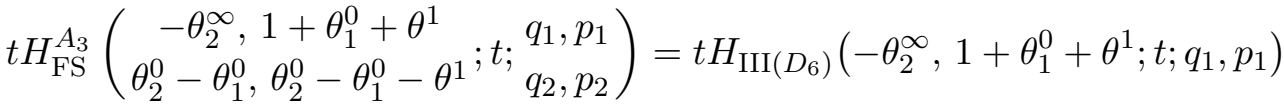

$$
\begin{aligned}
& +t H_{\mathrm{III}\left(D_{6}\right)}\left(\theta_{2}^{0}-\theta_{1}^{0}, \theta_{2}^{0}-\theta_{1}^{0}-\theta^{1} ; t ; q_{2}, p_{2}\right)-p_{1} p_{2}\left(q_{1} q_{2}+t\right) .
\end{aligned}
$$


Remark 3.1. Note that the expression of the Hamiltonian for the degenerate Fuji-Suzuki system of type $A_{3}$ is slightly different from that in [15]. Here we adopted the following expression:

$$
t H_{\mathrm{FS}}^{A_{3}}\left(\begin{array}{l}
\alpha, \beta \\
\gamma, \delta
\end{array} ; ; ; \begin{array}{l}
q_{1}, p_{1} \\
q_{2}, p_{2}
\end{array}\right)=t H_{\mathrm{III}\left(D_{6}\right)}\left(\alpha, \beta ; t ; q_{1}, p_{1}\right)+t H_{\mathrm{III}\left(D_{6}\right)}\left(\gamma, \delta ; t ; q_{2}, p_{2}\right)-p_{1} p_{2}\left(q_{1} q_{2}+t\right) .
$$

This Hamiltonian can be transformed into the expression of $H_{\mathrm{FS}}^{A_{3}}$ in [15] via the following canonical transformation:

$$
q_{2} \rightarrow \frac{t}{q_{2}}, \quad p_{2} \rightarrow-\frac{q_{2}}{t}\left(p_{2} q_{2}+\gamma\right) .
$$

\section{Singularity pattern $2+\frac{3}{2}$}

Spectral type $(1)_{2} 1,(11)(1)$. The Riemann scheme is given as

$$
\left(\begin{array}{cccc}
x=0 & \overbrace{1}^{x} \theta_{1}^{0} & \overbrace{\sqrt{t}} & \theta_{1}^{\infty} / 2 \\
0 & \theta_{2}^{0} & -\sqrt{t} & \theta_{1}^{\infty} / 2 \\
0 & 0 & 0 & \theta_{2}^{\infty}
\end{array}\right),
$$

and the Fuchs-Hukuhara relation is written as $\theta_{1}^{0}+\theta_{2}^{0}+\theta_{1}^{\infty}+\theta_{2}^{\infty}=0$. The Lax pair is expressed as

$$
\frac{\partial Y}{\partial x}=\left(\frac{A_{2}}{x^{2}}+\frac{A_{1}}{x}+A_{0}\right) Y, \quad \frac{\partial Y}{\partial t}=\left(\frac{1}{t} A_{0} x+B_{0}\right) Y,
$$

where

$$
\begin{aligned}
& A_{0}=\left(\begin{array}{lll}
0 & t & 0 \\
0 & 0 & 0 \\
0 & 0 & 0
\end{array}\right) \\
& A_{1}=\left(\begin{array}{ccc}
\left(p_{1}+p_{2}\right) q_{1} & q_{1} & p_{2} q_{1} \\
1 & -p_{1} q_{1}-p_{2} q_{2}+\theta_{1}^{0} & 1 \\
p_{2}\left(q_{2}-q_{1}\right)+\theta_{2}^{0}+\theta_{2}^{\infty} & q_{2}-q_{1} & p_{2}\left(q_{2}-q_{1}\right)+\theta_{2}^{0}
\end{array}\right) \text {, } \\
& A_{2}=\left(\begin{array}{ccc}
0 & 0 & 0 \\
p_{1}+p_{2} & 1 & p_{2} \\
0 & 0 & 0
\end{array}\right) \\
& B_{0}=\frac{1}{t}\left(\begin{array}{ccc}
\left(p_{1}+p_{2}\right) q_{1} & 0 & 0 \\
1 & -p_{1} q_{1}-p_{2} q_{2}+\theta_{1}^{0} & 1 \\
p_{2}\left(q_{2}-q_{1}\right)+\theta_{2}^{0}+\theta_{2}^{\infty} & 0 & p_{2} q_{2}+\theta_{2}^{0}
\end{array}\right) .
\end{aligned}
$$

The Hamiltonian is given by

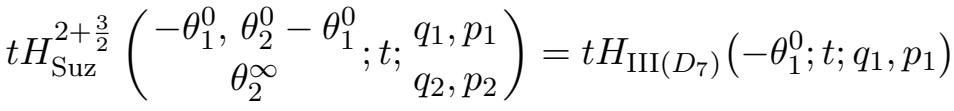

$$
\begin{aligned}
& +t H_{\mathrm{III}\left(D_{7}\right)}\left(\theta_{2}^{0}-\theta_{1}^{0} ; t ; q_{2}, p_{2}\right)+p_{2} q_{1}\left(p_{1}\left(q_{1}+q_{2}\right)+\theta_{2}^{\infty}\right)-q_{1} .
\end{aligned}
$$

\section{Singularity pattern $\frac{4}{3}+1+1$}

Spectral type $(1)_{3}, 21,111$. The Riemann scheme is given by

$$
\left(\begin{array}{cccc}
x=0 & x=1 & \overbrace{t^{\frac{1}{3}}} & \theta_{1}^{\infty} / 3 \\
0 & 0 & \left(\frac{1}{3}\right) \\
\theta_{1}^{0} & 0 & \omega t^{\frac{1}{3}} & \theta_{1}^{\infty} / 3 \\
\theta_{2}^{0} & \theta^{1} & \omega^{2} t^{\frac{1}{3}} & \theta_{1}^{\infty} / 3
\end{array}\right),
$$


and the Fuchs-Hukuhara relation is written as $\theta_{1}^{0}+\theta_{2}^{0}+\theta^{1}+\theta_{1}^{\infty}=0$. The Lax pair is expressed as

$$
\frac{\partial Y}{\partial x}=\left(\frac{A_{0}}{x}+\frac{A_{1}}{x-1}+A_{\infty}\right) Y, \quad \frac{\partial Y}{\partial t}=\left(\frac{1}{t} A_{\infty} x+B_{0}\right) Y
$$

Here

$$
\begin{aligned}
& A_{0}=\left(\begin{array}{ccc}
0 & 0 & 0 \\
-q_{2} & \theta_{1}^{0} & p_{1} q_{1}-\theta_{1}^{0}+\theta_{2}^{0} \\
q_{1}-q_{2} & 0 & \theta_{2}^{0}
\end{array}\right), \quad A_{\infty}=\left(\begin{array}{ccc}
0 & 1 & 0 \\
0 & 0 & 0 \\
0 & 0 & 0
\end{array}\right), \\
& A_{1}=\left(\begin{array}{c}
-p_{2} \\
1 \\
1
\end{array}\right)\left(\begin{array}{llc}
q_{2} & p_{1} q_{1}+p_{2} q_{2}+\frac{t}{q_{1}}-\theta_{1}^{0}+\theta_{2}^{0}+\theta^{1} & -p_{1} q_{1}-\frac{t}{q_{1}}+\theta_{1}^{0}-\theta_{2}^{0}
\end{array}\right), \\
& B_{0}=\frac{1}{t}\left(\begin{array}{ccc}
-p_{2} q_{2}-1 & 0 \\
0 & p_{1} q_{1}+p_{2} q_{2}+\frac{t}{q_{1}}+\theta_{2}^{0}+\theta^{1} & 0 \\
q_{1} & 0 & p_{1} q_{1}+p_{2}\left(q_{1}+q_{2}\right)+\theta_{2}^{0}+\theta^{1}
\end{array}\right) .
\end{aligned}
$$

The Hamiltonian is given by

$$
\begin{aligned}
& t H_{\mathrm{Suz}}^{2+\frac{3}{2}}\left(\begin{array}{c}
\theta_{2}^{0}-\theta_{1}^{0}+\theta^{1}+1, \theta_{2}^{0}+\theta^{1}+1 \\
\theta_{2}^{0}-\theta_{1}^{0}
\end{array} ; ; \begin{array}{l}
q_{1}, p_{1} \\
q_{2}, p_{2}
\end{array}\right)=t H_{\mathrm{III}\left(D_{7}\right)}\left(\theta_{2}^{0}-\theta_{1}^{0}+\theta^{1}+1 ; t ; q_{1}, p_{1}\right) \\
& \quad+t H_{\mathrm{III}\left(D_{7}\right)}\left(\theta_{2}^{0}+\theta^{1}+1 ; t ; q_{2}, p_{2}\right)+p_{2} q_{1}\left(p_{1}\left(q_{1}+q_{2}\right)+\theta_{2}^{0}-\theta_{1}^{0}\right)-q_{1} .
\end{aligned}
$$

\section{Singularity pattern $\frac{3}{2}+\frac{3}{2}$}

Spectral type $(1)_{2} 1,(1)_{2} 1$. The Riemann scheme is given by

$$
\left(\begin{array}{cccc}
x=0\left(\frac{1}{2}\right) & \overbrace{0}^{x} & \overbrace{\sqrt{t}} & \theta_{1}^{\infty} / 2 \\
1 & \theta^{0} / 2 & -\sqrt{t} & \theta_{1}^{\infty} / 2 \\
-1 & \theta^{0} / 2 & 0 & \theta_{2}^{\infty}
\end{array}\right),
$$

and the Fuchs-Hukuhara relation is written as $\theta^{0}+\theta_{1}^{\infty}+\theta_{2}^{\infty}=0$. The Lax pair is expressed as

$$
\frac{\partial Y}{\partial x}=\left(\frac{A_{2}}{x^{2}}+\frac{A_{1}}{x}+A_{0}\right) Y, \quad \frac{\partial Y}{\partial t}=\left(\frac{1}{t} A_{0} x+B_{0}\right) Y
$$

where

$$
\begin{array}{rlrl}
A_{0} & =\left(\begin{array}{lll}
0 & t & 0 \\
0 & 0 & 0 \\
0 & 0 & 0
\end{array}\right), & A_{1}=\left(\begin{array}{ccc}
-p_{2} q_{2} & 0 & -t p_{2} \\
1 & -p_{1} q_{1}+p_{2} q_{2}-\theta_{1}^{\infty} & q_{1} \\
p_{1} & 1 & p_{1} q_{1}-\theta_{2}^{\infty}
\end{array}\right) \\
A_{2}=\left(\begin{array}{ccc}
0 & 0 & 0 \\
q_{2} / t & 0 & 1 \\
0 & 0 & 0
\end{array}\right), & B_{0}=\frac{1}{t}\left(\begin{array}{ccc}
-p_{2} q_{2}+\theta_{1}^{\infty} & 0 & 0 \\
1 & -p_{1} q_{1}+p_{2} q_{2} & q_{1} \\
p_{1} & 0 & -p_{1} q_{1}+p_{2} q_{2}
\end{array}\right) .
\end{array}
$$

The Hamiltonian is given by

$$
\begin{aligned}
& t H_{\mathrm{KFS}}^{\frac{3}{2}+\frac{3}{2}}\left(\theta_{1}^{\infty}-\theta_{2}^{\infty}, 1-\theta_{1}^{\infty} ; t ; \begin{array}{l}
q_{1}, p_{1} \\
q_{2}, p_{2}
\end{array}\right)=t H_{\mathrm{III}\left(D_{7}\right)}\left(\theta_{1}^{\infty}-\theta_{2}^{\infty} ; t ; q_{1}, p_{1}\right) \\
& \quad+t H_{\mathrm{III}\left(D_{7}\right)}\left(1-\theta_{1}^{\infty} ; t ; q_{2}, p_{2}\right)-p_{1} q_{1} p_{2} q_{2}-t\left(p_{1} p_{2}+p_{1}+p_{2}\right) .
\end{aligned}
$$




\section{Singularity pattern $2+\frac{4}{3}$}

Spectral type $(1)_{3},(11)(1)$. The Riemann scheme is given by

$$
\left(\begin{array}{cccc}
x=0 & \overbrace{1}^{x} \theta_{2}^{0} & \overbrace{-t^{\frac{1}{3}}} & \theta_{1}^{\infty} / 3 \\
0 & \theta_{1}^{0} & -\omega t^{\frac{1}{3}} & \theta_{1}^{\infty} / 3 \\
0 & 0 & -\omega^{2} t^{\frac{1}{3}} & \theta_{1}^{\infty} / 3
\end{array}\right),
$$

and the Fuchs-Hukuhara relation is written as $\theta_{1}^{0}+\theta_{2}^{0}+\theta_{1}^{\infty}=0$. The Lax pair is expressed as

$$
\frac{\partial Y}{\partial x}=\left(\frac{A_{2}}{x^{2}}+\frac{A_{1}}{x}+A_{0}\right) Y, \quad \frac{\partial Y}{\partial t}=\left(\frac{1}{t} A_{0} x+B_{0}\right) Y
$$

where

$$
\begin{aligned}
A_{0}=\left(\begin{array}{lll}
0 & 1 & 0 \\
0 & 0 & 0 \\
0 & 0 & 0
\end{array}\right), & A_{1}=\left(\begin{array}{ccc}
-p_{2} q_{2} & -p_{2} & -p_{1} p_{2} \\
0 & -p_{1} q_{1}+p_{2} q_{2}+\theta_{2}^{0} & 1 \\
t & q_{1} & p_{1} q_{1}+\theta_{1}^{0}
\end{array}\right) \\
A_{2}=\left(\begin{array}{ccc}
0 & 0 & 0 \\
q_{2} & 1 & p_{1} \\
0 & 0 & 0
\end{array}\right), & B_{0}=\frac{1}{t}\left(\begin{array}{ccc}
-p_{2} q_{2}-1 & 0 & 0 \\
0 & -p_{1} q_{1}+p_{2} q_{2}+\theta_{2}^{0} & 1 \\
t & 0 & p_{1} q_{1}+\theta_{1}^{0}
\end{array}\right) .
\end{aligned}
$$

The Hamiltonian is given by

$$
\begin{aligned}
& t H_{\mathrm{KFS}}^{\frac{3}{2}+\frac{3}{2}}\left(\theta_{1}^{0}-\theta_{2}^{0}, \theta_{2}^{0}+1 ; t ; \begin{array}{c}
q_{1}, p_{1} \\
q_{2}, p_{2}
\end{array}\right)=t H_{\mathrm{III}\left(D_{7}\right)}\left(\theta_{1}^{0}-\theta_{2}^{0} ; t ; q_{1}, p_{1}\right) \\
& \quad+t H_{\mathrm{III}\left(D_{7}\right)}\left(\theta_{2}^{0}+1 ; t ; q_{2}, p_{2}\right)-p_{1} q_{1} p_{2} q_{2}-t\left(p_{1} p_{2}+p_{1}+p_{2}\right) .
\end{aligned}
$$

\section{Singularity pattern $\frac{3}{2}+\frac{4}{3}$}

Spectral type $(1)_{3},(1)_{2} 1$. The Riemann scheme is given by

$$
\left(\begin{array}{cccc}
\overbrace{0}^{x=0} \begin{array}{c}
0 \\
0
\end{array} & \overbrace{-t^{\frac{1}{3}}} & \theta_{1}^{\infty} / 3 \\
1 & \theta^{0} / 2 & -\omega t^{\frac{1}{3}} & \theta_{1}^{\infty} / 3 \\
-1 & \theta^{0} / 2 & -\omega^{2} t^{\frac{1}{3}} & \theta_{1}^{\infty} / 3
\end{array}\right)
$$

and the Fuchs-Hukuhara relation is written as $\theta^{0}+\theta_{1}^{\infty}=0$. The Lax pair is expressed as

$$
\frac{\partial Y}{\partial x}=\left(\frac{A_{2}}{x^{2}}+\frac{A_{1}}{x}+A_{0}\right) Y, \quad \frac{\partial Y}{\partial t}=\left(\frac{1}{t} A_{0} x+B_{0}\right) Y
$$

where

$$
\begin{aligned}
A_{0}=\left(\begin{array}{lll}
0 & 1 & 0 \\
0 & 0 & 0 \\
0 & 0 & 0
\end{array}\right), & A_{1}=\left(\begin{array}{ccc}
-p_{2} q_{2} & 0 & -p_{2} \\
0 & -p_{1} q_{1}+p_{2} q_{2}-\theta_{1}^{\infty} & q_{1} \\
t / q_{1} & 1 & p_{1} q_{1}
\end{array}\right), \\
A_{2}=\left(\begin{array}{lll}
0 & 0 & 0 \\
q_{2} & 0 & 1 \\
0 & 0 & 0
\end{array}\right), & B_{0}=\frac{1}{t}\left(\begin{array}{ccc}
-p_{2} q_{2}-1 & 0 & 0 \\
0 & -p_{1} q_{1}+p_{2} q_{2}-\theta_{1}^{\infty} & q_{1} \\
t / q_{1} & 0 & -p_{1} q_{1}+p_{2} q_{2}-\theta_{1}^{\infty}
\end{array}\right) .
\end{aligned}
$$


The Hamiltonian is given by

$$
\begin{aligned}
& t H_{\mathrm{KFS}}^{\frac{3}{2}+\frac{4}{3}}\left(\theta_{1}^{\infty} ; t ; \begin{array}{c}
q_{1}, p_{1} \\
q_{2}, p_{2}
\end{array}\right)=t H_{\mathrm{III}\left(D_{7}\right)}\left(\theta_{1}^{\infty} ; t ; q_{1}, p_{1}\right) \\
& \quad+t H_{\mathrm{III}\left(D_{7}\right)}\left(1-\theta_{1}^{\infty} ; t ; q_{2}, p_{2}\right)-p_{1} q_{1} p_{2} q_{2}-t\left(\frac{p_{2}}{q_{1}}+p_{1}+p_{2}\right) .
\end{aligned}
$$

\section{Singularity pattern $\frac{4}{3}+\frac{4}{3}$}

Spectral type $(1)_{3},(1)_{3}$. The Riemann scheme is given by

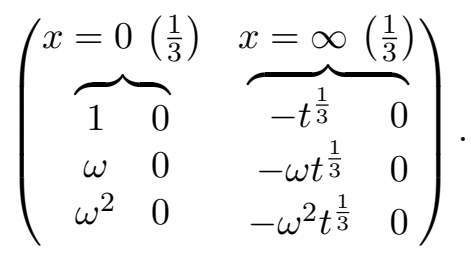

The Lax pair is expressed as

$$
\frac{\partial Y}{\partial x}=\left(\frac{A_{2}}{x^{2}}+\frac{A_{1}}{x}+A_{0}\right) Y, \quad \frac{\partial Y}{\partial t}=\left(\frac{1}{t} A_{0} x+B_{0}\right) Y,
$$

where

$$
\begin{aligned}
A_{0}=\left(\begin{array}{lll}
0 & 1 & 0 \\
0 & 0 & 0 \\
0 & 0 & 0
\end{array}\right), & A_{1}=\left(\begin{array}{ccc}
p_{2} q_{2}+1 & 0 & \frac{q_{1} q_{2}}{t} \\
0 & p_{1} q_{1}-p_{2} q_{2} & t \\
1 & -1 / q_{1} & -p_{1} q_{1}
\end{array}\right) \\
A_{2}=\left(\begin{array}{ccc}
0 & 0 & 0 \\
-t / q_{2} & 0 & 0 \\
0 & 0 & 0
\end{array}\right), & B_{0}=\frac{1}{t}\left(\begin{array}{ccc}
p_{2} q_{2} & 0 & 0 \\
0 & p_{1} q_{1}-p_{2} q_{2} & t \\
1 & 0 & -p_{1} q_{1}-1
\end{array}\right) .
\end{aligned}
$$

The Hamiltonian is given by

$$
\begin{aligned}
& t H_{\mathrm{KFS}}^{\frac{4}{3}+\frac{4}{3}}\left(t ; \begin{array}{l}
q_{1}, p_{1} \\
q_{2}, p_{2}
\end{array}\right)=t H_{\mathrm{III}\left(D_{8}\right)}\left(t ; q_{1}, p_{1}\right) \\
& \quad+t H_{\mathrm{III}\left(D_{8}\right)}\left(t ; q_{2}, p_{2}\right)-p_{1} q_{1} p_{2} q_{2}+\left(\frac{q_{1} q_{2}}{t}+q_{1}+q_{2}\right) .
\end{aligned}
$$

\section{Laplace transform}

In the degeneration scheme of the Garnier system and the Fuji-Suzuki system, we can see that the same Hamiltonian appears in several places. The linear systems corresponding to the same Hamiltonian can be transformed into one another by the Laplace transform. In this section, we present the correspondences through the Laplace transform.

It is known that a linear system (2.1) with $r_{\infty} \leq 1$ can canonically be written in the following form [34]:

$$
\frac{\mathrm{d} Y}{\mathrm{~d} x}=\left(Q(x-T)^{-1} P+S\right) Y .
$$

This system transforms into

$$
\frac{\mathrm{d} Y}{\mathrm{~d} x}=\left(-P(x-S)^{-1} Q-T\right) Y
$$


by the Laplace transform $x \mapsto-\mathrm{d} / \mathrm{d} x, \mathrm{~d} / \mathrm{d} x \mapsto x$. The correspondence between (4.1) and (4.2) is known as the Harnad duality when both $T$ and $S$ are semisimple [6], while here we do not impose the semisimplicity of $T$ or $S$.

Using this, we have the following correspondences of linear systems:

$$
\begin{array}{ll}
H_{\mathrm{Gar}}^{2+2+1}: & (1)(1),(1)(1), 11 \leftrightarrow(1)_{2}(1), 21,21, \\
H_{\mathrm{Gar}}^{3+2}: & ((1))((1)),(1)(1) \leftrightarrow((1))_{3}, 21,21, \\
H_{\mathrm{Gar}}^{2+\frac{3}{2}+1}: & (1)_{2},(1)(1), 11 \leftrightarrow(1)_{2}(1),(2)(1), \\
H_{\mathrm{Gar}}^{3+\frac{3}{2}}: & ((1))((1)),(1)_{2} \leftrightarrow((1))_{3},(2)(1), \\
H_{\mathrm{FS}}^{A_{3}}: & (11)(1),(11)(1) \leftrightarrow(1)_{2} 1,21,111, \\
H_{\mathrm{Suz}}^{2+\frac{3}{2}}: & (1)_{2} 1,(11)(1) \leftrightarrow(1)_{3}, 21,111, \\
H_{\mathrm{KFS}}^{\frac{3}{2}+\frac{3}{2}}: & (1)_{2} 1,(1)_{2} 1 \leftrightarrow(1)_{3},(11)(1) .
\end{array}
$$

Here we indicated which spectral types correspond to $\infty$.

When $r_{\infty}$ is greater than one, the correspondence through the Laplace transform is somewhat complicated. However, by using the method described in [15], we obtain the following correspondences:

$$
\begin{array}{ll}
H_{\mathrm{Gar}}^{3+2}: & ((1))^{\infty}((1)),(1)(1) \leftrightarrow((1))^{\infty}(1)_{2}, 21, \\
H_{\mathrm{Gar}}^{4+1}: & (((1)))^{\infty}(((1))), 11 \leftrightarrow(((1)))_{2} 1,21, \\
H_{\mathrm{Gar}}^{\frac{5}{2}+2}: & (((1)))_{2},(1)(1) \leftrightarrow\left(((1))^{\infty}\right)(1)_{2}, \\
H_{\mathrm{Gar}}^{5}: & ((((1))))_{2}, 21 \leftrightarrow((((1))))^{\infty}((((1)))) .
\end{array}
$$

\section{Conclusion}

The degeneration scheme presented in this series of papers focus on linear equations. Thus the scheme is redundant in terms of Hamiltonians since it happens that a certain Hamiltonian appears in several places.

When we focus on Hamiltonians, the degeneration scheme can be reduced to the following scheme. This scheme shows the relationship among 40 Painlevé-type equations including those already known.

Remark 5.1. To determine whether the Hamiltonians given different names in this series of papers are actually different or not requires further consideration. Concerning this problem, we refer to [19], which is an attempt to characterize the four-dimensional Painlevé-type equations from an algebro-geometric point of view.

Remark 5.2. We think that there are no degenerations of linear systems other than what we considered in this series of papers, and hence we believe that there are no other four-dimensional Painlevé-type equations. However, further research is needed to show that the four-dimensional Painlevé-type equations obtained in this series of papers actually constitute a complete list. We

think that a way to classify the unramified linear equations with four accessory parameters gives a hint (see [7]). 


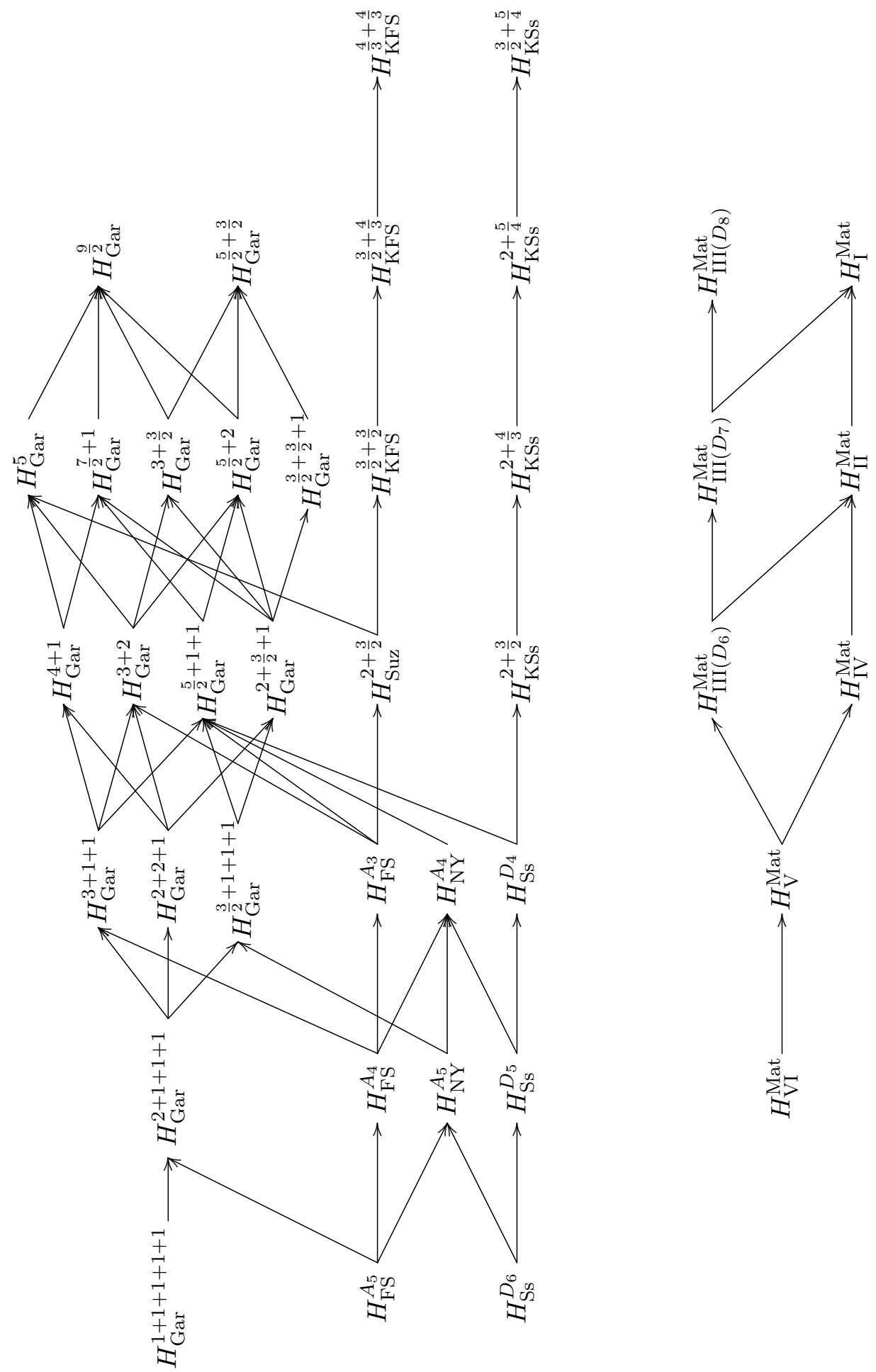

\section{A Data on degenerations}

In this appendix, we give explicit coordinate transformations used in degenerations. For example, for the linear system associated with $H_{\mathrm{Gar}}^{2+1+1+1}$, changing the variables and parameters $q_{i}$, $p_{i}, t_{i}, \theta_{i}^{*}, x, Y$ as shown in the table and taking the limit $\varepsilon \rightarrow 0$, we obtain the linear system associated with $H_{\text {Gar }}^{\frac{3}{2}+1+1+1}$. Also at this time, $\lim _{\varepsilon \rightarrow 0} \tilde{H}_{i}$ gives the Hamiltonian $H_{\text {Gar }, t_{i}}^{\frac{3}{2}+1+1}$.

The data that do not appear in the table below do not need to be changed. 


\section{A.1 degenerations of the Garnier system}

$2+1+1+1 \rightarrow 3 / 2+1+1+1$

$$
\begin{aligned}
& \theta_{1}^{\infty}=\tilde{\theta}_{1}^{\infty}+\varepsilon^{-1}, \quad \theta_{2}^{\infty}=-\varepsilon^{-1}, \quad t_{i}=\varepsilon \tilde{t}_{i}, \quad H_{t_{i}}=\varepsilon^{-1} \tilde{H}_{i}, \quad i=1,2, \\
& q_{1}=-\frac{1}{\varepsilon \tilde{q}_{1}}, \quad p_{1}=\varepsilon \tilde{q}_{1}\left(\tilde{p}_{1} \tilde{q}_{1}-\theta^{1}\right), \quad q_{2}=-\frac{1}{\varepsilon \tilde{q}_{2}}, \quad p_{2}=\varepsilon \tilde{q}_{2}\left(\tilde{p}_{2} \tilde{q}_{2}-\theta^{t}\right), \\
& x=\frac{\tilde{x}}{\tilde{t}_{1}}, \quad Y=t_{1} \tilde{\theta}_{1}^{\infty}+\varepsilon^{-1}\left(\begin{array}{rr}
1 & \\
& u
\end{array}\right)^{-1}\left(\begin{array}{rr}
0 & 1 \\
\varepsilon & 1
\end{array}\right) \tilde{Y} .
\end{aligned}
$$

$3+1+1 \rightarrow 5 / 2+1+1$

$$
\begin{aligned}
& \theta_{1}^{\infty}=\tilde{\theta}_{1}^{\infty}-\varepsilon^{-6}, \quad \theta_{2}^{\infty}=\varepsilon^{-6}, \quad t_{i}=\varepsilon^{-3}\left(\varepsilon^{4} \tilde{t}_{i}-2\right), \quad H_{t_{i}}=\varepsilon^{-1} \tilde{H}_{i}, \\
& q_{i}=\varepsilon^{-3}\left(\varepsilon^{2} \tilde{q}_{i}+1\right), \quad p_{i}=\varepsilon \tilde{p}_{i}, \quad i=1,2, \\
& x=-\varepsilon\left(\tilde{x}-\tilde{t}_{2}\right), \quad Y=e^{\varepsilon^{-2}\left(x-\tilde{t}_{2}\right)}\left(\begin{array}{cc}
\varepsilon^{-1} & -\varepsilon^{-3} \\
0 & -1
\end{array}\right) \tilde{Y} .
\end{aligned}
$$

$$
2+2+1 \rightarrow 2+3 / 2+1
$$

$\theta_{1}^{\infty}=\tilde{\theta}_{1}^{\infty}+\varepsilon^{-1}, \quad \theta_{2}^{\infty}=-\varepsilon^{-1}, \quad t_{1}=\varepsilon \tilde{t}_{1}, \quad t_{2}=-\varepsilon \tilde{t}_{2}$,

$H_{t_{1}}=\varepsilon^{-1} \tilde{H}_{1}, \quad H_{t_{2}}=-\varepsilon^{-1}\left(\tilde{H}_{2}-\frac{\tilde{p}_{2} \tilde{q}_{2}}{\tilde{t}_{2}}\right)$,

$q_{1}=-\frac{1}{\varepsilon \tilde{q}_{1}}, \quad p_{1}=\varepsilon \tilde{q}_{1}\left(\tilde{p}_{1} \tilde{q}_{1}-\theta^{1}\right), \quad q_{2}=\varepsilon \tilde{t}_{2} \tilde{p}_{2}, \quad p_{2}=-\frac{\tilde{q}_{2}}{\varepsilon \tilde{t}_{2}}$,

$x=\frac{\tilde{x}}{\tilde{t}_{1}}, \quad Y=t_{1} \tilde{\theta}_{1}^{\tilde{\theta}^{\infty}+\varepsilon^{-1}}\left(\begin{array}{ll}1 & \\ & u\end{array}\right)^{-1}\left(\begin{array}{ll}1 & -\varepsilon^{-1} \\ 0 & -\varepsilon^{-1}\end{array}\right) \tilde{Y}$.

$3 / 2+1+1+1 \rightarrow 5 / 2+1+1$

$$
\begin{aligned}
& \theta^{0}=-2 \varepsilon^{-3}, \quad \theta_{1}^{\infty}=\tilde{\theta}_{1}^{\infty}+2 \varepsilon^{-3}, \quad t_{i}=-\varepsilon^{-6}\left(\varepsilon^{2} \tilde{t}_{i}+1\right), \quad H_{t_{i}}=-\varepsilon^{4} \tilde{H}_{i}, \\
& q_{i}=\varepsilon^{-2} \tilde{q}_{i}+\varepsilon^{-3}, \quad p_{i}=\varepsilon^{2} \tilde{p}_{i}, \quad i=1,2, \\
& x=-\varepsilon^{-6}\left(\varepsilon^{2} \tilde{x}+1\right), \quad Y=e^{-\varepsilon^{-1} x}\left(\begin{array}{cc}
1 & -\varepsilon^{-1} \\
0 & \varepsilon^{2}
\end{array}\right) \tilde{Y} .
\end{aligned}
$$

$3 / 2+1+1+1 \rightarrow 2+3 / 2+1$

$$
\begin{aligned}
& \theta^{0}=-\varepsilon^{-1}, \quad \theta^{t}=\tilde{\theta}^{0}+\varepsilon^{-1}, \quad t_{2}=-\varepsilon \tilde{t}_{2}, \\
& q_{2}=\varepsilon \tilde{t}_{2} \tilde{p}_{2}, \quad p_{2}=-\frac{\tilde{q}_{2}}{\varepsilon \tilde{t}_{2}}, \quad H_{t_{2}}=-\varepsilon^{-1}\left(\tilde{H}_{2}-\frac{\tilde{p}_{2} \tilde{q}_{2}}{\tilde{t}_{2}}\right) .
\end{aligned}
$$

\section{$4+1 \rightarrow 7 / 2+1$}

$$
\begin{aligned}
& \theta_{1}^{\infty}=\tilde{\theta}_{1}^{\infty}-2 \varepsilon^{-15}, \quad \theta_{2}^{\infty}=2 \varepsilon^{-15}, \quad t_{1}=\varepsilon^{2} \tilde{t}_{1}-3 \varepsilon^{-10}, \quad t_{2}=-\varepsilon \tilde{t}_{2}+\varepsilon^{-5}, \\
& H_{t_{1}}=\varepsilon^{-2}\left(\tilde{H}_{1}-\frac{\varepsilon^{3}}{2} \tilde{q}_{1}\right), \quad H_{t_{2}}=-\varepsilon^{-1} \tilde{H}_{2}, \\
& q_{1}=\varepsilon \tilde{q}_{1}+\varepsilon^{-5}, \quad p_{1}=\frac{\varepsilon^{2}}{2}\left(\tilde{q}_{1}^{2}+\tilde{t}_{1}\right)+\varepsilon^{-1} \tilde{p}_{1}+\varepsilon^{-4} \tilde{q}_{1}-\varepsilon^{-10}, \\
& q_{2}=\varepsilon^{-2} \tilde{q}_{2}+\varepsilon^{-5}, \quad p_{2}=\varepsilon^{2} \tilde{p}_{2}, \\
& x=\varepsilon \tilde{x}, \quad Y=\exp \left(-\frac{1}{2 \varepsilon^{3}}\left(\tilde{x}-\tilde{t}_{2}\right)^{2}+\varepsilon^{-9}\left(\tilde{x}-\tilde{t}_{2}\right)-\varepsilon^{-3} \tilde{t}_{1}\right)\left(\begin{array}{cc}
1 & \varepsilon^{-3} \\
0 & \varepsilon^{2}
\end{array}\right) \tilde{Y} .
\end{aligned}
$$




\section{$3+2 \rightarrow 3+3 / 2$}

$$
\begin{aligned}
& \theta^{0}=2 \varepsilon^{-1}, \quad \theta_{1}^{\infty}=\tilde{\theta}_{1}^{\infty}-\varepsilon^{-1}, \quad \theta_{2}^{\infty}=\tilde{\theta}_{2}^{\infty}-\varepsilon^{-1}, \quad t_{1}=\sqrt{-1} \varepsilon \tilde{t}_{1}, \quad t_{2}=\sqrt{-1} \tilde{t}_{2}, \\
& H_{t_{1}}=\frac{1}{\sqrt{-1} \varepsilon}\left(\tilde{H}_{1}-\frac{\tilde{p}_{1} \tilde{q}_{1}+\tilde{p}_{2} \tilde{q}_{2}}{\tilde{t}_{1}}+\frac{1}{\tilde{t}_{1} \tilde{p}_{2}}\right), \quad H_{t_{2}}=\frac{1}{\sqrt{-1}}\left(\tilde{H}_{2}+\tilde{p}_{2} \tilde{q}_{1}\right), \\
& q_{1}=\frac{\tilde{t}_{1}}{f}, \quad p_{1}=-\frac{f}{\tilde{t}_{1}}\left(\tilde{p}_{1} \tilde{q}_{1}+\tilde{p}_{2} \tilde{q}_{2}+\tilde{\theta}_{1}^{\infty}+\frac{1}{\varepsilon}-\frac{1}{\tilde{p}_{2}}\right), \\
& q_{2}=\sqrt{-1} f \tilde{p}_{2}, \quad p_{2}=\sqrt{-1}\left(\frac{1}{f}\left(\tilde{q}_{2}+\frac{\tilde{p}_{1} \tilde{q}_{1}+2 \tilde{\theta}_{1}^{\infty}}{\tilde{p}_{2}}-\frac{1}{\tilde{p}_{2}^{2}}\right)+\tilde{p}_{2} \tilde{q}_{1}\right), \\
& x=\sqrt{-1} \tilde{x}, \quad Y=x^{\varepsilon^{-1}}\left(\begin{array}{cc}
1 & 0 \\
0 & u
\end{array}\right)^{-1}\left(\begin{array}{cc}
1 & 0 \\
0 & \frac{1}{\sqrt{-1} \tilde{p}_{2}^{2} f}
\end{array}\right)\left(\begin{array}{cc}
1 & 0 \\
0 & \tilde{u}
\end{array}\right) \tilde{Y},
\end{aligned}
$$

where

$$
f=\tilde{q}_{1}-\frac{\tilde{t}_{2}}{\tilde{p}_{2}}+\frac{\tilde{p}_{1}}{\tilde{p}_{2}^{2}} .
$$

The new gauge parameter $\tilde{u}$ satisfies the following equations:

$$
\frac{1}{\tilde{u}} \frac{\partial \tilde{u}}{\partial \tilde{t}_{1}}=-\frac{2}{\tilde{t}_{1}}\left(\tilde{p}_{1} \tilde{q}_{1}+\tilde{p}_{2} \tilde{q}_{2}+\tilde{\theta}_{1}^{\infty}\right), \quad \frac{1}{\tilde{u}} \frac{\partial \tilde{u}}{\partial \tilde{t}_{2}}=-2 \tilde{p}_{2} \tilde{q}_{1} .
$$

$3+2 \rightarrow 5 / 2+2$

$$
\begin{aligned}
& \theta_{1}^{\infty}=\tilde{\theta}_{1}^{\infty}-\varepsilon^{-6}, \quad \theta_{2}^{\infty}=\varepsilon^{-6}, \quad t_{1}=\varepsilon \tilde{t}_{2}, t_{2}=\varepsilon \tilde{t}_{1}-2 \varepsilon^{-3}, \\
& H_{t_{1}}=\varepsilon^{-1} \tilde{H}_{2}-\frac{\tilde{p}_{2} \tilde{q}_{2}}{\varepsilon \tilde{t}_{2}}, \quad H_{t_{2}}=\varepsilon^{-1} \tilde{H}_{1}-\varepsilon \tilde{p}_{1}, \quad q_{1}=\varepsilon^{2} \tilde{t}_{2} \tilde{p}_{2}, \quad p_{1}=-\frac{\tilde{q}_{2}}{\varepsilon^{2} \tilde{t}_{2}}, \\
& q_{2}=\varepsilon^{-3}+\varepsilon^{-1} \tilde{q}_{1}+\varepsilon\left(\tilde{p}_{1}-\tilde{t}_{1}\right), \quad p_{2}=\varepsilon \tilde{p}_{1}, \\
& x=\varepsilon \tilde{x}, \quad Y=e^{\varepsilon^{-2}\left(\tilde{x}-\tilde{t}_{1}\right)}\left(\begin{array}{cc}
0 & 1 \\
\varepsilon^{5} & 1 / q_{2}
\end{array}\right) \tilde{Y} .
\end{aligned}
$$

$5 / 2+1+1 \rightarrow 7 / 2+1$

$$
\begin{aligned}
& \theta^{t_{1}}=\tilde{\theta}^{0}, \quad \theta^{t_{2}}=-2 \varepsilon^{-15}, \quad \theta_{1}^{\infty}=\tilde{\theta}_{1}^{\infty}+2 \varepsilon^{-15}, \\
& t_{1}=\varepsilon^{-4} \tilde{t}_{2}-2 \varepsilon^{-10}, \quad t_{2}=\varepsilon^{2} \tilde{t}_{1}-3 \varepsilon^{-10}, \quad H_{t_{1}}=\varepsilon^{4} \tilde{H}_{2}, \quad H_{t_{2}}=\varepsilon^{-2}\left(\tilde{H}_{1}-\frac{\varepsilon^{3}}{2} \tilde{q}_{1}\right), \\
& q_{1}=\varepsilon^{-2} \tilde{q}_{2}, \quad p_{1}=\varepsilon^{2} \tilde{p}_{2}, \quad q_{2}=\varepsilon \tilde{q}_{1}+\varepsilon^{-5}, \\
& p_{2}=\frac{\varepsilon^{2}}{2}\left(\tilde{q}_{1}^{2}+\tilde{t}_{1}\right)+\varepsilon^{-1} \tilde{p}_{1}+\varepsilon^{-4} \tilde{q}_{1}-\varepsilon^{-10}, \\
& x=-\varepsilon^{-4} \tilde{x}+\varepsilon^{-4} \tilde{t}_{2}-2 \varepsilon^{-10}, \quad Y=\exp \left(\varepsilon^{-5} x+\frac{\varepsilon^{5}}{2} x^{2}+\varepsilon^{-3} \tilde{t}_{1}\right)\left(\begin{array}{cc}
1 & 0 \\
0 & -\varepsilon^{2}
\end{array}\right) \tilde{Y} .
\end{aligned}
$$

$5 / 2+1+1 \rightarrow 5 / 2+2$

$$
\begin{aligned}
& \theta^{t_{1}}=\varepsilon^{-1}, \quad \theta^{t_{2}}=\tilde{\theta}^{0}-\varepsilon^{-1}, \quad t_{2}=\tilde{t}_{1}+\varepsilon \tilde{t}_{2}, \quad H_{t_{1}}=\tilde{H}_{1}-\varepsilon^{-1} \tilde{H}_{2}, \quad H_{t_{2}}=\varepsilon^{-1} \tilde{H}_{2}, \\
& q_{1}=\tilde{q}_{1}, \quad p_{1}=\tilde{p}_{1}-\varepsilon^{-1} \tilde{p}_{2}, \quad q_{2}=\tilde{q}_{1}+\varepsilon \tilde{q}_{2}, \quad p_{2}=\varepsilon^{-1} \tilde{p}_{2}, \\
& x=-\tilde{x}+t_{1}, \quad Y=\left(\begin{array}{cc}
1 & -q_{1} \\
0 & 1
\end{array}\right) \tilde{Y} .
\end{aligned}
$$


$2+3 / 2+1 \rightarrow 7 / 2+1$

$$
\begin{aligned}
& \theta^{0}=-3 \varepsilon^{-5}, \quad \theta_{1}^{\infty}=\tilde{\theta}_{1}^{\infty}+3 \varepsilon^{-5}, \quad \theta^{t_{1}}=\tilde{\theta}^{0}, \\
& t_{1}=-\varepsilon^{-8} \tilde{t}_{2}-\varepsilon^{-10}, \quad t_{2}=2 \varepsilon^{-11} \tilde{t}_{1}+2 \varepsilon^{-15}, \\
& H_{t_{1}}=-\varepsilon^{8}\left(\tilde{H}_{2}+\frac{\varepsilon^{2} \tilde{p}_{2} \tilde{q}_{2}+\varepsilon \tilde{p}_{2}}{1+\varepsilon^{2} \tilde{t}_{2}}\right), \quad H_{t_{2}}=\frac{\varepsilon^{11}}{2} \tilde{H}_{1}, \\
& q_{1}=\varepsilon^{-5}\left(1+\varepsilon^{2} \tilde{t}_{2}\right)\left(1+\varepsilon \tilde{q}_{2}\right), \quad p_{1}=\frac{\varepsilon^{4} \tilde{p}_{2}}{1+\varepsilon^{2} \tilde{t}_{2}}, \\
& q_{2}=\varepsilon^{-10}\left(1-\varepsilon^{2} \tilde{q}_{1}\right), \quad p_{2}=\frac{\varepsilon^{5}}{2}-\frac{\varepsilon^{7} \tilde{q}_{1}}{2}-\varepsilon^{8} \tilde{p}_{1}, \\
& x=t_{1}\left(1-\varepsilon^{2} \tilde{x}\right), \quad Y=\exp \left[\frac{\tilde{x}-\tilde{t}_{2}}{2 \varepsilon^{3}}-\frac{\tilde{t}_{1}}{\varepsilon}-\frac{1}{\varepsilon}\left(\frac{\left(\tilde{x}-\tilde{t}_{2}\right)^{2}}{4}-\frac{\tilde{t}_{2} \tilde{x}}{2}\right)\right]\left(\begin{array}{cc}
1 & \varepsilon^{-1} \\
0 & -\frac{1}{\varepsilon^{6} t_{1}}
\end{array}\right) \tilde{Y} .
\end{aligned}
$$

$2+3 / 2+1 \rightarrow 3+3 / 2$

$$
\begin{aligned}
& \theta^{0}=\tilde{\theta}_{2}^{\infty}-\tilde{\theta}_{1}^{\infty}-\frac{1}{\varepsilon^{2}}, \quad \theta^{t_{1}}=\frac{1}{\varepsilon^{2}}, \quad \theta_{1}^{\infty}=2 \tilde{\theta}_{1}^{\infty}, \quad t_{1}=\varepsilon \tilde{t}_{1}, \quad t_{2}=\tilde{t}_{1} \tilde{t}_{2}+\frac{\tilde{t}_{1}}{\varepsilon}, \\
& H_{t_{1}}=\frac{1}{\varepsilon}\left(\tilde{H}_{1}-\frac{1}{\tilde{t}_{1}}\left(\tilde{t}_{2}+\frac{1}{\varepsilon}\right) \tilde{H}_{2}-\frac{\tilde{p}_{2} \tilde{q}_{2}}{\tilde{t}_{1}}-\frac{\tilde{p}_{2} \tilde{q}_{1}}{\varepsilon \tilde{t}_{1}}\right), \quad H_{t_{2}}=\frac{1}{\tilde{t}_{1}} \tilde{H}_{2}, \\
& q_{1}=\varepsilon \tilde{q}_{1}, \quad p_{1}=\frac{1}{\varepsilon}\left(\tilde{p}_{1}-\frac{\tilde{p}_{2}}{\varepsilon}\right), \quad q_{2}=\frac{\tilde{t}_{1} \tilde{p}_{2}}{\varepsilon}, \quad p_{2}=-\frac{1}{\tilde{t}_{1}}\left(\varepsilon \tilde{q}_{2}+\tilde{q}_{1}\right), \\
& x=\frac{\tilde{t}_{1}}{\tilde{x}}, \quad Y=\tilde{x}^{\tilde{\theta}_{1}^{\infty}} v\left(\begin{array}{cc}
-1 / \tilde{p}_{2} & 0 \\
-1 & 1 / \tilde{p}_{2}
\end{array}\right)\left(\begin{array}{cc}
1 & 0 \\
0 & \tilde{u}
\end{array}\right) \tilde{Y} .
\end{aligned}
$$

Here $\tilde{u}$ and $v$ satisfies

$$
\begin{aligned}
& \frac{1}{\tilde{u}} \frac{\partial \tilde{u}}{\partial \tilde{t}_{1}}=-\frac{2}{\tilde{t}_{1}}\left(\tilde{p}_{1} \tilde{q}_{1}+\tilde{p}_{2} \tilde{q}_{2}+\tilde{\theta}_{1}^{\infty}\right), \quad \frac{1}{\tilde{u}} \frac{\partial \tilde{u}}{\partial \tilde{t}_{2}}=-2 \tilde{p}_{2} \tilde{q}_{1}, \\
& \frac{1}{v} \frac{\partial v}{\partial \tilde{t}_{1}}=-\frac{1}{\tilde{t}_{1}}\left(\tilde{p}_{1} \tilde{q}_{1}+\tilde{p}_{2} \tilde{q}_{2}+2 \tilde{\theta}_{1}^{\infty}-\frac{1}{\tilde{p}_{2}}\right), \quad \frac{1}{v} \frac{\partial v}{\partial \tilde{t}_{2}}=-\frac{\tilde{p}_{1}}{\tilde{p}_{2}}-\tilde{p}_{2} \tilde{q}_{1}+\tilde{t}_{2} .
\end{aligned}
$$

$2+3 / 2+1 \rightarrow 5 / 2+2$

$$
\begin{aligned}
& \theta^{t_{1}}=2 \varepsilon^{-3}, \quad \theta_{1}^{\infty}=\tilde{\theta}_{1}^{\infty}-2 \varepsilon^{-3}, \quad t_{1}=\varepsilon^{-4} \tilde{t}_{1}+\varepsilon^{-6}, \quad t_{2}=-\varepsilon^{-4} \tilde{t}_{2}, \\
& H_{t_{1}}=\varepsilon^{4} \tilde{H}_{1}, \quad H_{t_{2}}=-\varepsilon^{4}\left(\tilde{H}_{2}-\frac{\tilde{p}_{2} \tilde{q}_{2}}{\tilde{t}_{2}}\right), \\
& q_{1}=\varepsilon^{-3}-\varepsilon^{-2} \tilde{q}_{1}, \quad p_{1}=1-\varepsilon^{2} \tilde{p}_{1}, \quad q_{2}=-\varepsilon^{-2} \tilde{t}_{2} \tilde{p}_{2}, \quad p_{2}=\varepsilon^{2} \frac{\tilde{q}_{2}}{\tilde{t}_{2}} \\
& x=\varepsilon^{-4} \tilde{x}, \quad Y=e^{\varepsilon^{-1}\left(\tilde{t}_{1}-\tilde{x}\right)}\left(\begin{array}{cc}
1 & 0 \\
0 & -\varepsilon^{8} t_{1}
\end{array}\right) \tilde{Y} .
\end{aligned}
$$

$2+3 / 2+1 \rightarrow 3 / 2+3 / 2+1$

$$
\begin{aligned}
& \theta^{0}=-2 \varepsilon^{-1}, \quad \theta_{1}^{\infty}=\tilde{\theta}_{1}^{\infty}+2 \varepsilon^{-1}, \quad t_{2}=\varepsilon \tilde{t}_{2}, \quad H_{t_{2}}=\varepsilon^{-1} \tilde{H}_{2}, \\
& q_{2}=-\tilde{q}_{2}, \quad p_{2}=-\tilde{p}_{2}-\frac{1}{\varepsilon \tilde{q}_{2}}, \quad Y=x^{-\varepsilon^{-1}} \tilde{Y} .
\end{aligned}
$$

$5 \rightarrow 9 / 2$

$$
\theta_{1}^{\infty}=-\frac{5}{\varepsilon^{28}}, \quad \theta_{2}^{\infty}=\frac{5}{\varepsilon^{28}}, \quad t_{1}=\frac{2 \tilde{t}_{1}}{3 \varepsilon^{5}}-\varepsilon^{3} \tilde{t}_{2}+\frac{160}{27 \varepsilon^{21}}, \quad t_{2}=\varepsilon^{2} \tilde{t}_{1}-\frac{5}{3 \varepsilon^{14}},
$$




$$
\begin{aligned}
& H_{t_{1}}=-\frac{1}{\varepsilon^{3}} \tilde{H}_{2}, \quad H_{t_{2}}=\frac{1}{\varepsilon^{2}}\left(\tilde{H}_{1}+\frac{2}{3 \varepsilon^{8}} \tilde{H}_{2}+\frac{\tilde{p}_{1}}{\varepsilon^{4}}\right), \\
& q_{1}=\varepsilon^{5} \tilde{q}_{2}-\varepsilon \tilde{p}_{1}-\frac{4}{3 \varepsilon^{7}}, \quad p_{1}=\frac{1}{\varepsilon^{5}}\left(\tilde{p}_{1}^{2}+\tilde{p}_{2}\right)+\frac{\tilde{q}_{2}}{\varepsilon^{9}}-\frac{\tilde{p}_{1}}{3 \varepsilon^{13}}+\frac{4}{3 \varepsilon^{21}}, \\
& q_{2}=-\frac{\tilde{p}_{1}}{\varepsilon^{6}}+\frac{1}{\varepsilon^{14}}, \quad p_{2}=\left(\tilde{q}_{1}-2 \tilde{p}_{1} \tilde{q}_{2}\right) \varepsilon^{6}+\left(-\tilde{p}_{2}+\tilde{t}_{1}\right) \varepsilon^{2}-\frac{2 \tilde{q}_{2}}{3 \varepsilon^{2}}+\frac{2 \tilde{p}_{1}}{3 \varepsilon^{6}}+\frac{4}{9 \varepsilon^{14}}, \\
& x=\varepsilon \tilde{x}+\frac{2}{3 \varepsilon^{7}}, \quad Y=\exp \left[\frac{\tilde{t}_{1} \tilde{x}+\frac{\tilde{x}^{3}}{3}-\tilde{t}_{2}}{\varepsilon^{4}}-\frac{\frac{\tilde{x}^{2}}{2}+\tilde{t}_{1}}{\varepsilon^{12}}+\frac{2 \tilde{x}}{\varepsilon^{20}}\right]\left(\begin{array}{cc}
1 & 0 \\
0 & u
\end{array}\right)^{-1}\left(\begin{array}{cc}
1 & -\varepsilon^{-4} \\
0 & -\varepsilon^{3}
\end{array}\right) \tilde{Y} .
\end{aligned}
$$

\section{$7 / 2+1 \rightarrow 9 / 2$}

$$
\begin{aligned}
& \theta^{0}=-\frac{2}{\varepsilon^{35}}, \quad \theta_{1}^{\infty}=\frac{2}{\varepsilon^{35}}, \quad t_{1}=\frac{\tilde{t}_{1}}{\varepsilon^{8}}+\frac{5}{3 \varepsilon^{28}}, \quad t_{2}=\varepsilon^{6} \tilde{t}_{1}-\varepsilon^{16} \tilde{t}_{2}-\frac{5}{3 \varepsilon^{14}}, \\
& H_{t_{1}}=\varepsilon^{8} \tilde{H}_{1}+\frac{\tilde{H}_{2}}{\varepsilon^{2}}, \quad H_{t_{2}}=-\frac{\tilde{H}_{2}}{\varepsilon^{16}}, \\
& q_{1}=\frac{\tilde{p}_{1}}{\varepsilon^{4}}-\frac{1}{3 \varepsilon^{14}}, \quad p_{1}=-\varepsilon^{4} \tilde{q}_{1}-\frac{\tilde{p}_{1}^{2}+\tilde{p}_{2}}{\varepsilon}+\frac{\tilde{q}_{2}}{\varepsilon^{6}}, \\
& q_{2}=-\varepsilon^{8} \tilde{q}_{2}-\varepsilon^{3} \tilde{p}_{1}-\frac{1}{\varepsilon^{7}}, \quad p_{2}=-\frac{\tilde{p}_{2}}{\varepsilon^{8}}-\frac{\tilde{p}_{1}}{\varepsilon^{18}}+\frac{1}{\varepsilon^{28}}, \\
& x=\frac{1}{\varepsilon^{14}}\left(\varepsilon^{10} \tilde{x}-1\right), \quad Y=\exp \left[\frac{\tilde{x}^{3}}{3 \varepsilon^{5}}+\frac{\tilde{x}^{2}}{2 \varepsilon^{15}}+\frac{\tilde{x}}{\varepsilon^{25}}\right]\left(\begin{array}{cc}
1 & 0 \\
0 & -\varepsilon^{2}
\end{array}\right) \tilde{Y} .
\end{aligned}
$$

\section{$3+3 / 2 \rightarrow 9 / 2$}

$$
\begin{aligned}
& \theta_{1}^{\infty}=-\frac{15}{8 \varepsilon^{14}}, \quad \theta_{2}^{\infty}=\frac{15}{8 \varepsilon^{14}}, \quad t_{1}=-\frac{\sqrt{2}}{\varepsilon^{35}}\left(1+\varepsilon^{8} \tilde{t}_{1}+\varepsilon^{12} \tilde{t}_{2}\right), \quad t_{2}=-\sqrt{2} \varepsilon \tilde{t}_{1}+\frac{5}{\sqrt{2} \varepsilon^{7}}, \\
& H_{t_{1}}=-\frac{\varepsilon^{23}}{\sqrt{2}}\left(\tilde{H}_{2}+\varepsilon^{12} \frac{\tilde{p}_{1} Q_{1}+\left(\tilde{p}_{2}-\tilde{t}_{1}\right) Q_{2}}{1+\varepsilon^{8} \tilde{t}_{1}+\varepsilon^{12} \tilde{t}_{2}}\right), \quad H_{t_{2}}=-\frac{1}{\sqrt{2} \varepsilon}\left(\tilde{H}_{1}-\frac{\tilde{H}_{2}}{\varepsilon^{4}}-\frac{5 \tilde{p}_{1}}{8 \varepsilon^{2}}+\tilde{q}_{2}\right), \\
& q_{1}=\sqrt{2}\left(1+\varepsilon^{8} \tilde{t}_{1}+\varepsilon^{12} \tilde{t}_{2}\right)\left(\frac{Q_{1}}{\varepsilon^{11}}-\frac{Q_{2}}{\varepsilon^{15}}\right), \quad p_{1}=\frac{\varepsilon^{11} \tilde{p}_{1}}{\sqrt{2}\left(1+\varepsilon^{8} \tilde{t}_{1}+\varepsilon^{12} \tilde{t}_{2}\right)}-\sqrt{2} \varepsilon^{7}, \\
& q_{2}=\frac{2\left(1+\varepsilon^{8} \tilde{t}_{1}+\varepsilon^{12} \tilde{t}_{2}\right) Q_{2}}{\varepsilon^{22}}, \quad p_{2}=\frac{\varepsilon^{18} \tilde{p}_{1}+\varepsilon^{22}\left(\tilde{p}_{2}-\tilde{t}_{1}\right)}{2\left(1+\varepsilon^{8} \tilde{t}_{1}+\varepsilon^{12} \tilde{t}_{2}\right)}-\frac{\varepsilon^{14}}{2}, \\
& x=\frac{\sqrt{2}}{\varepsilon^{7}\left(1-\varepsilon^{4} \tilde{x}\right)}, \\
& Y=\exp \left[\frac{3 \tilde{x}}{2 \varepsilon^{10}}+\frac{\tilde{x}^{2}+\frac{\tilde{t}_{1}}{2}}{\varepsilon^{6}}+\frac{-\tilde{t}_{1} \tilde{x}+\frac{\tilde{x}^{3}}{2}+\tilde{t}_{2}}{\varepsilon^{2}}\right]\left(\begin{array}{cc}
1 & 0 \\
0 & u
\end{array}\right)^{-1}\left(\begin{array}{cc}
1 & \varepsilon^{-2} \\
p_{2} q_{1} & q_{1}\left(\frac{p_{2}}{\varepsilon^{2}}-\frac{\sqrt{2}}{\varepsilon^{23} t_{1}}\right)
\end{array}\right) \tilde{Y},
\end{aligned}
$$

where

$$
Q_{1}=\tilde{q}_{1}+\frac{1}{8}\left(\frac{\tilde{p}_{1}}{\varepsilon^{6}}-\frac{3 \tilde{p}_{1}^{2}}{\varepsilon^{2}}-\frac{3 \tilde{p}_{2}}{\varepsilon^{2}}-\frac{2 \tilde{t}_{1}}{\varepsilon^{2}}-\frac{3}{\varepsilon^{10}}\right), \quad Q_{2}=\tilde{q}_{2}-\frac{3 \tilde{p}_{1}}{8 \varepsilon^{2}}+\frac{1}{8 \varepsilon^{6}}
$$

\section{$3+3 / 2 \rightarrow 5 / 2+3 / 2$}

$$
\begin{aligned}
& \theta_{1}^{\infty}=\varepsilon^{-6}, \quad \theta_{2}^{\infty}=-\varepsilon^{-6}, \quad t_{1}=\varepsilon \tilde{t}_{2}, \quad t_{2}=\varepsilon \tilde{t}_{1}-2 \varepsilon^{-3}, \\
& H_{t_{1}}=\varepsilon^{-1} \tilde{H}_{2}, \quad H_{t_{2}}=\varepsilon^{-1}\left(\tilde{H}_{1}+\tilde{q}_{1}\right), \\
& q_{1}=\varepsilon^{-1} \tilde{q}_{2}, \quad p_{1}=\frac{1}{\varepsilon \tilde{q}_{2}}\left(\tilde{p}_{1}-\tilde{q}_{1}^{2}-\tilde{t}_{1}+\varepsilon^{2}\left(\tilde{p}_{2} \tilde{q}_{2}-\tilde{p}_{1} \tilde{q}_{1}+\tilde{t}_{1} \tilde{q}_{1}\right)\right), \\
& q_{2}=\frac{\tilde{q}_{2}}{\varepsilon^{4}}\left(1-\varepsilon^{2} \tilde{q}_{1}\right), \quad p_{2}=-\frac{1}{\varepsilon^{2} \tilde{q}_{2}}\left(1+\varepsilon^{2} \tilde{q}_{1}+\varepsilon^{4}\left(\tilde{p}_{1}-\tilde{t}_{1}\right)\right),
\end{aligned}
$$




$$
x=\varepsilon \tilde{x}, \quad Y=\exp \left(\varepsilon^{-2}\left(\tilde{t}_{1}-\tilde{x}\right)\right)\left(\begin{array}{cc}
1 & 0 \\
0 & u
\end{array}\right)^{-1}\left(\begin{array}{cc}
0 & \varepsilon q_{1} \\
1 & \varepsilon p_{2} q_{1}
\end{array}\right) \tilde{Y} .
$$

$5 / 2+2 \rightarrow 9 / 2$

$$
\begin{aligned}
& \theta^{0}=-\frac{5}{\varepsilon^{21}}, \quad \theta_{1}^{\infty}=\frac{5}{\varepsilon^{21}}, \quad t_{1}=\frac{\tilde{t}_{1}}{\varepsilon^{2}}+\varepsilon^{4} \tilde{t}_{2}-\frac{5}{\varepsilon^{14}}, \quad t_{2}=-\frac{2 \tilde{t}_{1}}{\varepsilon^{23}}-\frac{2}{\varepsilon^{35}}, \\
& H_{t_{1}}=\frac{\tilde{H}_{2}}{\varepsilon^{4}}, \\
& H_{t_{2}}=\frac{\varepsilon^{23}}{2}\left[-\tilde{H}_{1}+\frac{\tilde{H}_{2}}{\varepsilon^{6}}+\frac{\varepsilon^{3}}{2\left(1+\varepsilon^{12} \tilde{t}_{1}\right)}\left\{\tilde{p}_{1}^{2}-\tilde{p}_{2}-2 \varepsilon^{3} \tilde{p}_{1} \tilde{q}_{2}+\varepsilon^{6}\left(\tilde{p}_{2}+\tilde{t}_{1}\right) \tilde{p}_{1}-2 \varepsilon^{9} \tilde{p}_{2} \tilde{q}_{2}\right\}\right] \text {, } \\
& q_{1}=\varepsilon^{8} \tilde{q}_{1}-\frac{\varepsilon^{5}}{2}\left(\tilde{p}_{1}^{2}+\tilde{p}_{2}+\tilde{t}_{1}\right)-\varepsilon^{2} \tilde{q}_{2}+\frac{\tilde{p}_{1}}{\varepsilon}+\frac{1}{\varepsilon^{7}}, \quad p_{1}=\frac{\tilde{p}_{1}}{\varepsilon^{8}}-\frac{2}{\varepsilon^{14}}, \\
& q_{2}=\frac{2\left(1+\varepsilon^{12} \tilde{t}_{1}\right)}{\varepsilon^{19}}\left(\tilde{q}_{2}-\frac{\tilde{p}_{1}}{2 \varepsilon^{3}}+\frac{1}{2 \varepsilon^{9}}\right), \quad p_{2}=\frac{\varepsilon^{19}}{2\left(1+\varepsilon^{12} \tilde{t}_{1}\right)}\left(\tilde{p}_{2}-\tilde{t}_{1}+\frac{\tilde{p}_{1}}{\varepsilon^{6}}-\frac{1}{\varepsilon^{12}}\right), \\
& x=\frac{1}{\varepsilon^{14}}\left(\varepsilon^{6} \tilde{x}-1\right), \quad Y=\exp \left[-\frac{\frac{\tilde{x}^{3}}{6}+\tilde{t}_{1} \tilde{x}}{\varepsilon^{3}}+\frac{\frac{\tilde{x}^{2}}{4}-\tilde{t}_{1}}{\varepsilon^{9}}+\frac{3 \tilde{x}}{2 \varepsilon^{15}}\right]\left(\begin{array}{cc}
1 & \varepsilon^{-3} \\
0 & \varepsilon^{4}
\end{array}\right) \tilde{Y} .
\end{aligned}
$$

$5 / 2+2 \rightarrow 5 / 2+3 / 2$

$$
\begin{aligned}
& \theta^{0}=2 \varepsilon^{-1}, \quad \theta_{1}^{\infty}=-2 \varepsilon^{-1}, \quad t_{2}=\varepsilon \tilde{t}_{2}, \quad H_{t_{2}}=\varepsilon^{-1} \tilde{H}_{2}, \\
& q_{2}=\tilde{q}_{2}, \quad p_{2}=\tilde{p}_{2}+\frac{1}{\varepsilon \tilde{q}_{2}}, \quad Y=\tilde{x}^{\varepsilon^{-1}} \tilde{Y} .
\end{aligned}
$$

$3 / 2+3 / 2+1 \rightarrow 5 / 2+3 / 2$

$$
\begin{aligned}
& \theta^{t_{1}}=2 \varepsilon^{-3}, \quad \theta_{1}^{\infty}=-2 \varepsilon^{-3}, \quad t_{1}=\varepsilon^{-4} \tilde{t}_{1}+\varepsilon^{-6}, \quad t_{2}=\varepsilon^{-4} \tilde{t}_{2}, \\
& H_{t_{1}}=\varepsilon^{4} \tilde{H}_{1}, \quad H_{t_{2}}=\varepsilon^{4}\left(\tilde{H}_{2}-\frac{\tilde{p}_{2} \tilde{q}_{2}}{\tilde{t}_{2}}\right), \\
& q_{1}=-\varepsilon^{-2} \tilde{q}_{1}+\varepsilon^{-3}, \quad p_{1}=1-\varepsilon^{2} \tilde{p}_{1}, \quad q_{2}=\frac{\tilde{t}_{2}}{\varepsilon^{2} \tilde{q}_{2}}, \quad p_{2}=-\frac{\varepsilon^{2} \tilde{p}_{2} \tilde{q}_{2}^{2}}{\tilde{t}_{2}}, \\
& x=\varepsilon^{-4} \tilde{x}, \quad Y=\exp \left(\varepsilon^{-1}\left(\tilde{t}_{1}-\tilde{x}\right)\right)\left(\begin{array}{cc}
1 & 0 \\
0 & -\varepsilon^{2}
\end{array}\right) \tilde{Y} .
\end{aligned}
$$

\section{A.2 degenerations of the Fuji-Suzuki system}

We first note that, concerning the following two linear systems, we adopt slightly different parametrizations from those in [15] for convenience of calculation.

As for the linear system of the spectral type (11)(1),21,111, the irregular singular point is moved to $x=\infty$, so that the Riemann scheme is given by

$$
\left(\begin{array}{cccc}
x=0 & x=1 & \multicolumn{1}{c}{x=\infty} \\
0 & 0 & \overbrace{t} & \theta_{1}^{\infty} \\
\theta_{1}^{0} & 0 & 0 & \theta_{2}^{\infty} \\
\theta_{2}^{0} & \theta^{1} & 0 & \theta_{3}^{\infty}
\end{array}\right),
$$

and the Fuchs-Hukuhara relation is written as $\theta_{1}^{0}+\theta_{2}^{0}+\theta^{1}+\theta_{1}^{\infty}+\theta_{2}^{\infty}+\theta_{3}^{\infty}=0$. The Lax pair is expressed as

$$
\frac{\partial Y}{\partial x}=\left(\frac{A_{0}}{x}+\frac{A_{1}}{x-1}+A_{\infty}\right) Y, \quad \frac{\partial Y}{\partial t}=\left(\frac{1}{t} A_{\infty} x+B_{0}\right) Y .
$$


Here

$$
\begin{aligned}
& A_{\xi}=U^{-1} P^{-1} \hat{A}_{\xi} P U, \quad \xi=0,1, \quad U=\operatorname{diag}(1, u, v), \\
& P=\left(\begin{array}{ccc}
1 & 0 & 0 \\
p_{2} q_{2}-\theta_{2}^{0}-\theta_{2}^{\infty} & 1 & 0 \\
p_{1} q_{1}-\theta_{3}^{\infty} & \frac{p_{1}\left(q_{1}-q_{2}\right)-\theta_{3}^{\infty}}{\theta_{3}^{\infty}-\theta_{2}^{\infty}} & 1
\end{array}\right), \\
& \hat{A}_{0}=\left(\begin{array}{ll}
1 & 0 \\
0 & 1 \\
0 & 0
\end{array}\right)\left(\begin{array}{ccc}
\theta_{1}^{0} & q_{2}-1 & q_{1}-1 \\
0 & \theta_{2}^{0} & p_{2}\left(q_{1}-q_{2}\right)+\theta_{2}^{0}+\theta_{2}^{\infty}
\end{array}\right) \\
& \hat{A}_{1}=\left(\begin{array}{c}
1 \\
p_{2} \\
p_{1}
\end{array}\right)\left(\begin{array}{lll}
p_{1} q_{1}+p_{2} q_{2}+\theta^{1} & -q_{2} & -q_{1}
\end{array}\right), \\
& A_{\infty}=\left(\begin{array}{ccc}
-t & 0 & 0 \\
0 & 0 & 0 \\
0 & 0 & 0
\end{array}\right), \quad B_{0}=\frac{1}{t}\left(\begin{array}{ccc}
0 & \left(A_{0}+A_{1}\right)_{12} & \left(A_{0}+A_{1}\right)_{13} \\
\left(A_{0}+A_{1}\right)_{21} & 0 & 0 \\
\left(A_{0}+A_{1}\right)_{31} & 0 & 0
\end{array}\right) .
\end{aligned}
$$

The Hamiltonian is given by

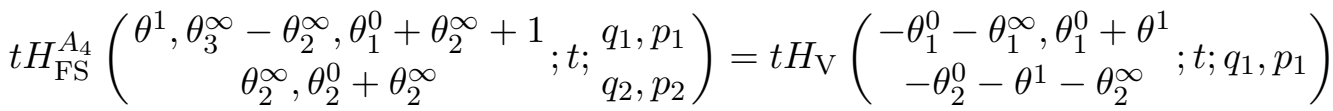

$$
\begin{aligned}
& +t H_{\mathrm{V}}\left(\begin{array}{c}
\theta_{2}^{0}+\theta^{1}+\theta_{2}^{\infty}, \theta_{1}^{0}-\theta_{2}^{0}+\theta^{1} ; t ; q_{2}, p_{2} \\
-\theta^{1}
\end{array}\right)+p_{1}\left(q_{2}-1\right)\left\{p_{2}\left(q_{1}+q_{2}\right)-\theta_{2}^{0}-\theta_{2}^{\infty}\right\} .
\end{aligned}
$$

The gauge parameters satisfy

$$
\frac{t}{u} \frac{\mathrm{d} u}{\mathrm{~d} t}=-p_{1} q_{2}-t q_{2}+\theta_{1}^{\infty}-\theta_{2}^{\infty}, \quad \frac{t}{v} \frac{\mathrm{d} v}{\mathrm{~d} t}=-t q_{1}+\theta_{1}^{\infty}-\theta_{3}^{\infty} .
$$

Concerning the linear system of the spectral type $(11)(1),(11)(1)$, as the expression of the Hamiltonian $H_{\mathrm{FS}}^{A_{3}}$ changed (see Remark 3.1), the parametrization of the linear system also changed slightly. The variables $x$ and $Y$ also changed in the obvious way.

As the result, the Riemann scheme is given by

$$
\left(\begin{array}{cccc}
\overbrace{1}^{x=0} & \theta_{1}^{0} & \overbrace{t} & \theta_{1}^{\infty} \\
0 & \theta_{2}^{0} & 0 & \theta_{2}^{\infty} \\
0 & 0 & 0 & \theta_{3}^{\infty}
\end{array}\right),
$$

and the Fuchs-Hukuhara relation is written as $\theta_{1}^{0}+\theta_{2}^{0}+\theta_{1}^{\infty}+\theta_{2}^{\infty}+\theta_{3}^{\infty}=0$. The Lax pair is expressed as

$$
\frac{\partial Y}{\partial x}=\left(\frac{A_{2}}{x^{2}}+\frac{A_{1}}{x}+A_{0}\right) Y, \quad \frac{\partial Y}{\partial t}=\left(\frac{1}{t} A_{0} x+B_{0}\right) Y,
$$

where

$$
\begin{aligned}
A_{\xi}=U^{-1} P^{-1} \hat{A}_{\xi} P U, & \xi=1,2, \quad P=\left(\begin{array}{ccc}
1 & 0 & 0 \\
-p_{2} & 1 & 0 \\
-q_{1} & \frac{q_{1} q_{2}-t}{\theta_{2}^{\infty}-\theta_{3}^{\infty}} & 1
\end{array}\right), \quad U=\operatorname{diag}(1, u, v), \\
A_{0}=\left(\begin{array}{ccc}
-t & 0 & 0 \\
0 & 0 & 0 \\
0 & 0 & 0
\end{array}\right), & \hat{A}_{1}=\left(\begin{array}{ccc}
p_{1} q_{1}-p_{2} q_{2}-\theta_{1}^{\infty} & -q_{2} & p_{1} \\
p_{2} q_{2}-\theta_{2}^{0}-\theta_{2}^{\infty} & p_{2} q_{2}-\theta_{2}^{\infty} & -p_{1} p_{2} \\
t & t & -p_{1} q_{1}-\theta_{3}^{\infty}
\end{array}\right),
\end{aligned}
$$




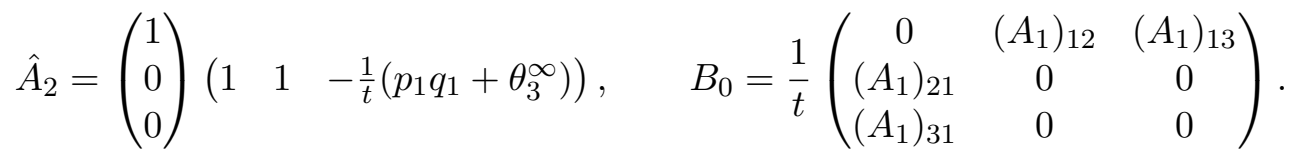

The Hamiltonian is given by

$$
\begin{aligned}
& t H_{\mathrm{FS}}^{A_{3}}\left(\begin{array}{c}
\alpha, \beta \\
\gamma, \delta
\end{array} ; t ; \begin{array}{c}
q_{1}, p_{1} \\
q_{2}, p_{2}
\end{array}\right)=t H_{\mathrm{III}\left(D_{6}\right)}\left(\theta_{3}^{\infty}, \theta_{3}^{\infty}-\theta_{1}^{\infty}+1 ; t ; q_{1}, p_{1}\right) \\
& \quad+t H_{\mathrm{III}\left(D_{6}\right)}\left(-\theta_{2}^{0}-\theta_{2}^{\infty}, \theta_{1}^{\infty}-\theta_{2}^{\infty} ; t ; q_{2}, p_{2}\right)-p_{1} p_{2}\left(q_{1} q_{2}+t\right) .
\end{aligned}
$$

The gauge parameters satisfy

$$
\frac{t}{u} \frac{\mathrm{d} u}{\mathrm{~d} t}=p_{1} q_{1}-2 p_{2} q_{2}+q_{2}, \quad \frac{t}{v} \frac{\mathrm{d} v}{\mathrm{~d} t}=2 p_{1} q_{1}-p_{2} q_{2}-q_{1}+1 .
$$

Below are the data for degenerations.

$\mathbf{2}+\mathbf{1}+\mathbf{1} \rightarrow \mathbf{2}+\mathbf{1}+\mathbf{1}$

$(1)(1)(1), 21,21 \rightarrow(1)_{2}(1), 21,21$

$$
\begin{aligned}
& \theta_{1}^{\infty}=\tilde{\theta}_{1}^{\infty}+\varepsilon^{-1}, \quad \theta_{3}^{\infty}=-\varepsilon^{-1}, \quad t_{2}=\varepsilon \tilde{t}_{2}, H_{t_{2}}=\varepsilon^{-1} \tilde{H}_{2}, \\
& q_{1}=\tilde{q}_{1}+\frac{\theta_{2}^{\infty}}{\tilde{p}_{1}}, \quad p_{1}=\tilde{p}_{1}, \quad q_{2}=\tilde{p}_{2}, \quad p_{2}=-\tilde{q}_{2}, \\
& Y=t_{2} \varepsilon^{-1} \operatorname{diag}(1, u, v)^{-1}\left(\begin{array}{ccc}
0 & \varepsilon & 0 \\
0 & 0 & \varepsilon p_{1} q_{1} \\
t_{2} q_{2} & \varepsilon q_{2}\left(p_{2} q_{2}-\theta_{3}^{\infty}\right) & 0
\end{array}\right) \tilde{Y} .
\end{aligned}
$$

$3+\mathbf{1} \rightarrow \mathbf{3}+\mathbf{1}$

$((1)(1))((1)), 21 \rightarrow((1))(1)_{2}, 21$

$$
\begin{aligned}
& \theta_{2}^{\infty}=\tilde{\theta}_{2}^{\infty}+\varepsilon^{-1}, \quad \theta_{3}^{\infty}=-\varepsilon^{-1}, \quad t_{1}=\tilde{t}_{2}-\varepsilon \tilde{t}_{1}, \quad t_{2}=\tilde{t}_{2}, \\
& H_{t_{1}}=-\frac{1}{\varepsilon}\left(\tilde{H}_{1}-\frac{\tilde{p}_{1} \tilde{q}_{1}}{\tilde{t}_{1}}\right), \quad H_{t_{2}}=\varepsilon^{-1} \tilde{H}_{1}+\tilde{H}_{2}-\frac{\tilde{p}_{1} \tilde{q}_{1}}{\varepsilon \tilde{t}_{1}}, \\
& q_{1}=\varepsilon \tilde{t}_{1} \tilde{p}_{1}+\tilde{q}_{2}, \quad p_{1}=-\frac{\tilde{q}_{1}}{\varepsilon \tilde{t}_{1}}, \quad q_{2}=\tilde{q}_{2}, \quad p_{2}=\tilde{p}_{2}+\frac{\tilde{q}_{1}}{\varepsilon \tilde{t}_{1}}, \\
& x=-\tilde{x}, \quad Y=\exp \left(\frac{1}{2} x^{2}-t_{2} x\right)\left(\begin{array}{ccc}
1 & \\
& u & \\
& & v
\end{array}\right)^{-1}\left(\begin{array}{ccc}
1 & 0 & 0 \\
0 & 1 & \frac{q_{1}}{\left(t_{1}-t_{2}\right) q_{2}} \\
0 & 0 & -\frac{q_{1}}{\left(t_{1}-t_{2}\right) q_{2}}
\end{array}\right) \tilde{Y} .
\end{aligned}
$$

$3+\mathbf{1} \rightarrow 5 / 2+1$

$((1)(1))((1)), 21 \rightarrow(((1)))_{2} 1,21$

$$
\begin{aligned}
& \theta_{1}^{\infty}=\tilde{\theta}_{1}^{\infty}-\varepsilon^{-6}, \quad \theta_{2}^{\infty}=\varepsilon^{-6}, \quad \theta_{3}^{\infty}=\tilde{\theta}_{2}^{\infty}, \\
& t_{1}=\varepsilon \tilde{t}_{1}-2 \varepsilon^{-3}, \quad t_{2}=-\varepsilon^{-1} \tilde{t}_{2}-\varepsilon^{-3}, \\
& q_{1}=-\varepsilon \tilde{p}_{1}, \quad p_{1}=\varepsilon^{-1} \tilde{q}_{1}-\varepsilon^{-3}, \quad q_{2}=\varepsilon^{-1} \tilde{q}_{2}, \quad p_{2}=\varepsilon \tilde{p}_{2}, \\
& H_{t_{1}}=\varepsilon^{-1} \tilde{H}_{1}, \quad H_{t_{2}}=-\varepsilon \tilde{H}_{2}, \\
& x=\varepsilon \tilde{x}, \quad Y=\exp \left(\frac{1}{2} x^{2}-t_{2} x-\varepsilon^{-2} \tilde{t}_{1}\right)\left(\begin{array}{ccc}
1 & & \\
& u & \\
& & v
\end{array}\right) \quad\left(\begin{array}{cccc}
0 & -1 & 0 \\
1 / \varepsilon & -p_{1} & 0 \\
0 & 0 & \varepsilon
\end{array}\right) \tilde{Y} .
\end{aligned}
$$


$2+1+1 \rightarrow 3+1$

$(1)_{2}(1), 21,21 \rightarrow((1))(1)_{2}, 21$

$$
\begin{aligned}
& \theta^{1}=\tilde{\theta}_{1}^{\infty}+\varepsilon^{-2}, \quad \theta_{1}^{\infty}=\tilde{\theta}_{2}^{\infty}, \quad \theta_{2}^{\infty}=-\varepsilon^{-2}, \quad t_{1}=\varepsilon^{-2}-\varepsilon^{-1} \tilde{t}_{2}, \quad t_{2}=\varepsilon^{-1} \tilde{t}_{1}, \\
& H_{t_{1}}=-\varepsilon \tilde{H}_{2}, \quad H_{t_{2}}=\varepsilon\left(\tilde{H}_{1}-\frac{\tilde{p}_{1} \tilde{q}_{1}}{\tilde{t}_{1}}\right), \quad q_{1}=\left(1+\frac{\tilde{q}_{1}}{\varepsilon\left(\tilde{q}_{1} \tilde{q}_{2}+\tilde{t}_{1}\right)}\right)^{-1}, \\
& p_{1}=-\left(\tilde{p}_{1} \tilde{q}_{1}-\tilde{p}_{2} \tilde{q}_{2}-\theta^{0}\right)\left(\frac{\tilde{q}_{1}}{\varepsilon\left(\tilde{q}_{1} \tilde{q}_{2}+\tilde{t}_{1}\right)}+2+\frac{\varepsilon\left(\tilde{q}_{1} \tilde{q}_{2}+\tilde{t}_{1}\right)}{\tilde{q}_{1}}\right)-\frac{\tilde{q}_{1} \tilde{q}_{2}+\tilde{t}_{1}}{\varepsilon \tilde{q}_{1}}-\varepsilon^{-2}, \\
& q_{2}=-\tilde{t}_{1} \frac{\tilde{q}_{1}\left(\tilde{p}_{1} \tilde{q}_{1}-\theta^{0}-\tilde{\theta}_{1}^{\infty}\right)+\tilde{t}_{1} \tilde{p}_{2}}{\tilde{q}_{1}\left(\tilde{q}_{1} \tilde{q}_{2}+\tilde{t}_{1}\right)}, \quad p_{2}=1+\frac{\tilde{q}_{1} \tilde{q}_{2}}{\tilde{t}_{1}}, \\
& x=\varepsilon \tilde{x}, \quad Y=f\left(\begin{array}{ccc}
\varepsilon t_{2} p_{2} q_{2} & -t_{2} & \frac{1}{\varepsilon p_{2}}\left(\left(q_{1}-1\right)\left(\varepsilon^{-2}-p_{1} q_{1}\right)+\theta^{1}\right)+\frac{q_{2}}{\varepsilon\left(q_{1}-1\right)} \\
\varepsilon t_{2}^{2} p_{2} & 0 & \frac{t_{2}}{\varepsilon\left(q_{1}-1\right)} \\
\varepsilon t_{2}^{2} p_{2}\left(q_{1}-1\right) & 0 & 0
\end{array}\right) \tilde{Y},
\end{aligned}
$$

where $f$ satisfies

$$
\frac{1}{f} \frac{\partial f}{\partial \tilde{t}_{1}}=-\tilde{p}_{1} \tilde{q}_{1}-1, \quad \frac{1}{f} \frac{\partial f}{\partial \tilde{t}_{2}}=-\tilde{q}_{2}-\tilde{t}_{2} .
$$

$2+1+1 \rightarrow 5 / 3+1+1$

$(1)_{2}(1), 21,21 \rightarrow((1))_{3}, 21,21$

$$
\begin{aligned}
& \theta_{1}^{\infty}=\tilde{\theta}_{1}^{\infty}+\varepsilon^{-2}, \quad \theta_{2}^{\infty}=-\varepsilon^{-2}, \quad t_{1}=-\varepsilon \tilde{t}_{1}\left(1+\varepsilon \tilde{t}_{2}\right), \quad t_{2}=\varepsilon^{-1} \tilde{t}_{1}, \\
& H_{t_{1}}=-\frac{1}{\varepsilon^{2} \tilde{t}_{1}} \tilde{H}_{2}, \quad H_{t_{2}}=\varepsilon \tilde{H}_{1}-\frac{1+\varepsilon \tilde{t}_{2}}{\tilde{t}_{1}} \tilde{H}_{2}-\frac{\varepsilon \tilde{p}_{1} \tilde{q}_{1}}{\tilde{t}_{1}}, \\
& q_{1}=1+\frac{\tilde{q}_{1} \tilde{q}_{2}}{\tilde{t}_{1}}+\frac{\tilde{q}_{1}}{\varepsilon \tilde{t}_{1}}, \quad p_{1}=\varepsilon \tilde{t}_{1}\left(\tilde{p}_{1}-\frac{\tilde{p}_{2} \tilde{q}_{2}+\theta^{0}}{\tilde{q}_{1}}\right)+\frac{\tilde{t}_{1}}{\varepsilon \tilde{q}_{1}}, \\
& q_{2}=\varepsilon \tilde{t}_{1}\left(\tilde{p}_{1}-\frac{\tilde{p}_{2} \tilde{q}_{2}+\theta^{0}}{\tilde{q}_{1}}\right)+\tilde{t}_{1} \frac{1-\varepsilon \tilde{p}_{2}}{\varepsilon \tilde{q}_{1}}, \quad p_{2}=1+\frac{\tilde{q}_{1} \tilde{q}_{2}}{\tilde{t}_{1}}, \\
& Y=\tilde{t}_{1}^{\varepsilon^{-2}} e^{\varepsilon^{-1} \tilde{t}_{2}-\frac{1}{2} \tilde{t}_{2}{ }^{2}}\left(\begin{array}{ccc}
1 & -1 / t_{1} & 0 \\
0 & 1 & \frac{q_{1}-p_{2}}{\varepsilon^{2} t_{2} q_{1}} \\
0 & 0 & \frac{q_{1}-p_{2}}{\varepsilon^{2} t_{2}}
\end{array}\right) \tilde{Y} .
\end{aligned}
$$

$2+1+1 \rightarrow 2+2$

$(1)_{2}(1), 21,21 \rightarrow(1)_{2}(1),(2)(1)$

$$
\begin{aligned}
& \theta^{0}=\tilde{\theta}^{0}-\varepsilon^{-1}, \quad \theta^{1}=\varepsilon^{-1}, \quad t_{1}=\varepsilon \tilde{t}_{1}, \quad t_{2}=-\varepsilon \tilde{t}_{2}, \\
& H_{t_{1}}=\varepsilon^{-1} \tilde{H}_{1}, \quad H_{t_{2}}=-\varepsilon^{-1}\left(\tilde{H}_{2}-\frac{\tilde{p}_{2} \tilde{q}_{2}}{\tilde{t}_{2}}\right), \\
& q_{1}=-\frac{1}{\varepsilon \tilde{q}_{1}}, \quad p_{1}=\varepsilon \tilde{q}_{1}\left(\tilde{p}_{1} \tilde{q}_{1}+\theta_{2}^{\infty}\right), \quad q_{2}=\varepsilon \tilde{t}_{2} \tilde{p}_{2}, \quad p_{2}=-\frac{\tilde{q}_{2}}{\varepsilon \tilde{t}_{2}}, \\
& x=\varepsilon^{-1} \tilde{x}, \quad Y=\left(\begin{array}{ccc}
1 & 0 & 0 \\
0 & \varepsilon & 0 \\
0 & 0 & \varepsilon q_{1}
\end{array}\right) \tilde{Y} .
\end{aligned}
$$

$2+\mathbf{2} \rightarrow \mathbf{2}+\mathbf{2}$

$(1)(1)(1),(2)(1) \rightarrow(1)_{2}(1),(2)(1)$

$$
\theta_{1}^{\infty}=\tilde{\theta}_{1}^{\infty}-\varepsilon^{-1}, \quad \theta_{3}^{\infty}=\varepsilon^{-1}, \quad t_{2}=\varepsilon \tilde{t}_{2}, \quad H_{t_{2}}=\varepsilon^{-1}\left(\tilde{H}_{2}-\frac{\tilde{p}_{2} \tilde{q}_{2}}{\tilde{t}_{2}}\right),
$$




$$
\begin{aligned}
& q_{1}=\tilde{q}_{1}+\frac{\theta_{2}^{\infty}}{\tilde{p}_{1}}, \quad p_{1}=\tilde{p}_{1}, \quad q_{2}=\frac{\tilde{t}_{2}}{\tilde{q}_{2}}, \quad p_{2}=-\frac{\tilde{q}_{2}\left(\tilde{p}_{2} \tilde{q}_{2}-\varepsilon^{-1}\right)}{\tilde{t}_{2}}, \\
& Y=\tilde{t}_{2}^{-\varepsilon^{-1}} \frac{p_{2}}{t_{2}} \cdot \operatorname{diag}(1, u, v)^{-1} \cdot\left(\begin{array}{ccc}
0 & \varepsilon q_{2} & 0 \\
0 & 0 & \varepsilon p_{1} q_{2} \\
t_{2} & \varepsilon p_{2} q_{2} & 0
\end{array}\right) \tilde{Y} .
\end{aligned}
$$

$4 \rightarrow 4$

$(((1)(1)))(((1))) \rightarrow(((1)))(1)_{2}$

$$
\begin{aligned}
& \theta_{2}^{\infty}=\tilde{\theta}_{2}^{\infty}+\varepsilon^{-1}, \quad \theta_{3}^{\infty}=-\varepsilon^{-1}, \quad t_{1}=\tilde{t}_{1}, t_{2}=\tilde{t}_{1}+\varepsilon \tilde{t}_{2}, \\
& H_{t_{1}}=\tilde{H}_{1}-\varepsilon^{-1} \tilde{H}_{2}, \quad H_{t_{2}}=\varepsilon^{-1} \tilde{H}_{2}, \\
& q_{1}=\tilde{q}_{1}, \quad p_{1}=\tilde{p}_{1}-\varepsilon^{-1} \tilde{p}_{2}, \quad q_{2}=\tilde{q}_{1}+\varepsilon \tilde{q}_{2}, \quad p_{2}=\varepsilon^{-1} \tilde{p}_{2}, \\
& x=-\tilde{x}, \quad Y=\exp \left(-\frac{x^{3}}{3}-t_{2} x\right)\left(\begin{array}{ccc}
\varepsilon & 0 & 0 \\
0 & 0 & 1 \\
0 & -\varepsilon & -1
\end{array}\right) \tilde{Y} .
\end{aligned}
$$

\section{$\mathbf{3}+\mathbf{1} \rightarrow \mathbf{4}$}

$((1))(1)_{2}, 21 \rightarrow(((1)))(1)_{2}$

$$
\begin{aligned}
& \theta^{0}=-\varepsilon^{-6}, \quad \theta_{1}^{\infty}=\tilde{\theta}_{1}^{\infty}+\varepsilon^{-6}, \quad t_{1}=\varepsilon \tilde{t}_{2}, \quad t_{2}=\varepsilon \tilde{t}_{1}-2 \varepsilon^{-3}, \\
& H_{t_{1}}=\varepsilon^{-1}\left(\tilde{H}_{2}-\frac{\tilde{p}_{2} \tilde{q}_{2}}{\tilde{t}_{2}}\right), \quad H_{t_{2}}=\varepsilon^{-1} \tilde{H}_{1}, \\
& q_{1}=\varepsilon^{2} \tilde{t}_{2} \tilde{p}_{2}, \quad p_{1}=-\frac{\tilde{q}_{2}}{\varepsilon^{2} \tilde{t}_{2}}, \quad q_{2}=\varepsilon^{-1} \tilde{q}_{1}+\varepsilon^{-3}, \quad p_{2}=\varepsilon \tilde{p}_{1}, \\
& x=\varepsilon^{-1} \tilde{x}+\varepsilon^{-3}, \quad Y=\exp \left(-\varepsilon^{-2} \tilde{t}_{1}\right)\left(\begin{array}{ccc}
1 & 0 & 0 \\
0 & \varepsilon^{-1} & 0 \\
0 & 0 & \tilde{t}_{2}
\end{array}\right) \tilde{Y} .
\end{aligned}
$$

$\mathbf{3}+\mathbf{1} \rightarrow \mathbf{7} / \mathbf{3}+\mathbf{1}$

$((1))(1)_{2}, 21 \rightarrow((((1))))_{3}, 21$

$$
\begin{aligned}
& \theta_{1}^{\infty}=\tilde{\theta}_{1}^{\infty}-3 \varepsilon^{-4}, \quad \theta_{2}^{\infty}=3 \varepsilon^{-4}, \quad t_{1}=\varepsilon^{-3} \tilde{t}_{1}+\varepsilon^{-4} \tilde{t}_{2}+\varepsilon^{-6}, \quad t_{2}=\tilde{t}_{2}-3 \varepsilon^{-2}, \\
& H_{t_{1}}=\varepsilon^{3} \tilde{H}_{1}, \quad H_{t_{2}}=-\varepsilon^{-1} \tilde{H}_{1}+\tilde{H}_{2} \\
& q_{1}=\varepsilon^{-3} \tilde{q}_{1}+\varepsilon^{-4}, \quad p_{1}=\varepsilon^{3} \tilde{p}_{1}-\varepsilon^{2} \tilde{q}_{2}, \quad q_{2}=\tilde{q}_{2}, \quad p_{2}=\tilde{p}_{2}-\varepsilon^{-1} \tilde{q}_{1}-2 \varepsilon^{-2}, \\
& x=\varepsilon \tilde{x}, \quad Y=\exp \left(\varepsilon^{-1} \tilde{x}+\varepsilon^{-1} \tilde{t}_{1}-\varepsilon^{-2} \tilde{t}_{2}\right)\left(\begin{array}{ccc}
0 & -\varepsilon & 0 \\
0 & \varepsilon^{-1}-\varepsilon p_{2}-\varepsilon^{3} q_{1} & 1 \\
\varepsilon^{-1} & -\varepsilon^{-3} & -\varepsilon^{-2}
\end{array}\right) \tilde{Y} \text {. }
\end{aligned}
$$

$\mathbf{5} / \mathbf{2}+\mathbf{1} \rightarrow \mathbf{7} / \mathbf{3}+\mathbf{1}$

$(((1)))_{2} 1,21 \rightarrow((((1))))_{3}, 21$

$$
\begin{aligned}
& \theta_{1}^{\infty}=\tilde{\theta}_{1}^{\infty}+\varepsilon^{-12}, \quad \theta_{2}^{\infty}=-\varepsilon^{-12}, \quad t_{1}=-\varepsilon \tilde{t}_{1}+\frac{\tilde{t}_{2}}{\varepsilon^{2}}+\frac{3}{4 \varepsilon^{8}}, \quad t_{2}=-\varepsilon^{2} \tilde{t}_{2}+\frac{3}{2 \varepsilon^{4}}, \\
& H_{t_{1}}=-\varepsilon^{-1} \tilde{H}_{1}, \quad H_{t_{2}}=-\varepsilon^{-5} \tilde{H}_{1}-\varepsilon^{-2} \tilde{H}_{2}-\varepsilon^{-2} \tilde{q}_{2}, \\
& q_{1}=-\frac{\tilde{q}_{1}}{\varepsilon}-\frac{1}{2 \varepsilon^{4}}, \quad p_{1}=-\varepsilon \tilde{p}_{1}-\frac{\tilde{q}_{2}}{\varepsilon^{2}}, \quad q_{2}=\varepsilon^{2} \tilde{q}_{2}-\varepsilon^{8}, \quad p_{2}=\frac{\tilde{p}_{2}-\tilde{t}_{2}}{\varepsilon^{2}}+\frac{\tilde{q}_{1}}{\varepsilon^{5}}+\frac{1}{\varepsilon^{8}}, \\
& x=-\varepsilon \tilde{x}, \quad Y=\exp \left(\frac{\tilde{x}}{\varepsilon^{3}}+\frac{\tilde{t}_{1}}{2 \varepsilon^{3}}+\frac{\tilde{t}_{2}}{2 \varepsilon^{6}}\right)\left(\begin{array}{ccc}
\varepsilon^{3} & \varepsilon q_{1}+\frac{1}{2 \varepsilon^{3}} & 1 \\
0 & \varepsilon & 0 \\
0 & -\varepsilon p_{2}-\frac{q_{1}}{\varepsilon^{3}}-\frac{1}{2 \varepsilon^{7}} & -\varepsilon^{-4}
\end{array}\right) \tilde{Y} .
\end{aligned}
$$


$5 / 3+1+1 \rightarrow 2+5 / 3$

$((1))_{3}, 21,21 \rightarrow((1))_{3},(2)(1)$

$$
\begin{aligned}
& \theta^{0}=-\varepsilon^{-1}, \quad \theta^{1}=\tilde{\theta}^{0}+\varepsilon^{-1}, \quad t_{1}=\varepsilon \tilde{t}_{1}, \\
& H_{t_{1}}=\frac{1}{\varepsilon}\left(\tilde{H}_{1}-\frac{\tilde{p}_{1} \tilde{q}_{1}+\tilde{p}_{2} \tilde{q}_{2}}{\tilde{t}_{1}}\right), \quad H_{t_{2}}=\tilde{H}_{2}+\tilde{p}_{2} \tilde{q}_{1}, \\
& q_{1}=\frac{\tilde{t}_{1}}{\tilde{q}_{1}}, \quad p_{1}=-\frac{\tilde{q}_{1}}{\varepsilon \tilde{t}_{1}}\left(\varepsilon \tilde{p}_{1} \tilde{q}_{1}+\varepsilon \tilde{p}_{2} \tilde{q}_{2}+1\right), \quad q_{2}=-\tilde{p}_{2} \tilde{q}_{1}, \quad p_{2}=-\tilde{p}_{2} \tilde{q}_{1}+\frac{\tilde{q}_{2}}{\tilde{q}_{1}}+\tilde{t}_{2}, \\
& x=\frac{\tilde{x}}{\varepsilon \tilde{t}_{1}}, \quad Y=\tilde{t}_{1}-\tilde{\theta}^{0}\left(\begin{array}{ccc}
1 & 0 & 0 \\
0 & \varepsilon \tilde{t}_{1} & 0 \\
0 & 0 & \varepsilon^{2} \tilde{t}_{1}^{2}
\end{array}\right) \tilde{Y} .
\end{aligned}
$$

$\mathbf{2}+\mathbf{2} \rightarrow \mathbf{4}$

$(1)_{2}(1),(2)(1) \rightarrow(((1)))(1)_{2}$

$$
\begin{aligned}
& \theta^{0}=\tilde{\theta}_{1}^{\infty}+2 \varepsilon^{-3}, \quad \theta_{1}^{\infty}=\tilde{\theta}_{2}^{\infty}, \quad \theta_{2}^{\infty}=-2 \varepsilon^{-3}, \quad t_{1}=\varepsilon^{-4} \tilde{t}_{1}+\varepsilon^{-6}, \quad t_{2}=\varepsilon^{-4} \tilde{t}_{2}, \\
& H_{t_{1}}=\varepsilon^{4} \tilde{H}_{1}, \quad H_{t_{2}}=\varepsilon^{4}\left(\tilde{H}_{2}+\frac{\tilde{p}_{1}}{\tilde{t}_{2} \tilde{p}_{2}}-\frac{\tilde{p}_{2} \tilde{q}_{2}}{\tilde{t}_{2}}\right), \quad q_{1}=\varepsilon^{-3}-\varepsilon^{-2}\left(\tilde{q}_{1}+\frac{1}{\tilde{p}_{2}}\right), \\
& p_{1}=1-\varepsilon^{2} \tilde{p}_{1}, \quad q_{2}=-\varepsilon^{-2} \tilde{t}_{2} \tilde{p}_{2}, \quad p_{2}=\frac{\varepsilon^{2}}{\tilde{t}_{2}}\left(\tilde{q}_{2}-\frac{\tilde{\theta}_{2}^{\infty}}{\tilde{p}_{2}}-\frac{\tilde{p}_{1}}{\tilde{p}_{2}^{2}}\right), \\
& x=-\varepsilon^{4}\left(\tilde{x}+\varepsilon^{-1}\right), \quad Y=f\left(\begin{array}{ccc}
0 & -1 / t_{2} & \frac{p_{1}-1}{q_{2}} \\
0 & 0 & 1 \\
-\frac{q_{2}}{\varepsilon t_{2}} & 0 & 0
\end{array}\right) \tilde{Y} .
\end{aligned}
$$

Here $f$ satisfies

$$
\frac{1}{f} \frac{\partial f}{\partial \tilde{t}_{1}}=-\tilde{q}_{1}, \quad \frac{1}{f} \frac{\partial f}{\partial \tilde{t}_{2}}=\frac{\tilde{p}_{2} \tilde{q}_{2}+1}{\tilde{t}_{2}} .
$$

$2+2 \rightarrow 2+5 / 3$

$(1)_{2}(1),(2)(1) \rightarrow((1))_{3},(2)(1)$

$$
\begin{aligned}
& \theta_{1}^{\infty}=\tilde{\theta}_{1}^{\infty}+\varepsilon^{-2}, \quad \theta_{2}^{\infty}=-\varepsilon^{-2}, \quad t_{1}=\varepsilon \tilde{t}_{1}, \quad t_{2}=\tilde{t}_{1} \tilde{t}_{2}+\varepsilon^{-1} \tilde{t}_{1}, \\
& H_{t_{1}}=\varepsilon^{-1}\left(\tilde{H}_{1}-\frac{1}{\tilde{t}_{1}}\left(\tilde{t}_{2}+\varepsilon^{-1}\right) \tilde{H}_{2}-\frac{\tilde{p}_{1} \tilde{q}_{1}}{\tilde{t}_{1}}-\frac{\tilde{p}_{2} \tilde{q}_{1}}{\varepsilon \tilde{t}_{1}}\right), \quad H_{t_{2}}=\frac{1}{\tilde{t}_{1}} \tilde{H}_{2}, \\
& q_{1}=\varepsilon\left(\tilde{q}_{1}-\varepsilon \tilde{q}_{2}\right), \quad p_{1}=-\frac{\tilde{p}_{2}}{\varepsilon^{2}}, \quad q_{2}=\frac{\tilde{t}_{1}}{\varepsilon}\left(\tilde{p}_{2}+\varepsilon \tilde{p}_{1}\right), \quad p_{2}=-\frac{\tilde{q}_{1}}{\tilde{t}_{1}}, \\
& x=\frac{\tilde{x}}{\tilde{t}_{1}}, \quad Y=\tilde{t}_{1}^{\varepsilon^{-2}+1-\theta^{0}}\left(\begin{array}{ccc}
-\frac{\varepsilon}{\tilde{t}_{1}} & 1 / \tilde{t}_{1} & 0 \\
0 & -\varepsilon & 1 \\
0 & 0 & 1
\end{array}\right) \tilde{Y} .
\end{aligned}
$$

$2+1+1 \rightarrow 3 / 2+1+1$

$(11)(1), 21,111 \rightarrow(1)_{2} 1,21,111$

$$
\begin{aligned}
& \theta_{1}^{0}=\tilde{\theta}^{0}-\varepsilon^{-1}, \quad \theta_{2}^{0}=\varepsilon^{-1}, \quad t=-\varepsilon \tilde{t}, \quad H=-\varepsilon^{-1} \tilde{H}, \\
& q_{1}=1-\tilde{p}_{1}-\tilde{p}_{2}, \quad p_{1}=\tilde{q}_{2}, \quad q_{2}=1-\tilde{p}_{1}, \quad p_{2}=\tilde{q}_{1}-\tilde{q}_{2} .
\end{aligned}
$$

$2+2 \rightarrow 2+3 / 2$

$(11)(1),(11)(1) \rightarrow(1)_{2} 1,(11)(1)$

$$
\theta_{1}^{\infty}=\tilde{\theta}_{1}^{\infty}+\varepsilon^{-1}, \quad \theta_{2}^{\infty}=-\varepsilon^{-1}, \quad \theta_{3}^{\infty}=\tilde{\theta}_{2}^{\infty}, \quad t=\varepsilon \tilde{t}, \quad H=\varepsilon^{-1} \tilde{H}-\frac{\tilde{p}_{1} \tilde{q}_{1}}{\varepsilon \tilde{t}},
$$




$$
\begin{aligned}
& q_{1}=\frac{\tilde{t}}{\tilde{q}_{1}}, \quad p_{1}=-\frac{\tilde{q}_{1}}{\tilde{t}}\left(\tilde{p}_{1} \tilde{q}_{1}+\tilde{\theta}_{2}^{\infty}\right), \quad q_{2}=-\frac{1}{\tilde{p}_{2}}, \quad p_{2}=-\tilde{p}_{2}\left(\tilde{p}_{2} \tilde{q}_{2}-\varepsilon^{-1}+\theta_{2}^{0}\right), \\
& Y=t^{\varepsilon^{-1}} U^{-1} P^{-1}\left(\begin{array}{ccc}
0 & 1 & 0 \\
-1 / q_{2} & 0 & -1 / q_{2} \\
\varepsilon q_{1} & 0 & 0
\end{array}\right) \tilde{Y} .
\end{aligned}
$$

$3 / 2+1+1 \rightarrow 2+3 / 2$

$(1)_{2} 1,21,111 \rightarrow(1)_{2} 1,(11)(1)$

$$
\begin{aligned}
& \theta_{1}^{0}=\tilde{\theta}_{1}^{0}-\varepsilon^{-1}, \quad \theta^{1}=\varepsilon^{-1}, \quad t=-\varepsilon \tilde{t}, \quad H=-\varepsilon^{-1} \tilde{H}+\frac{\tilde{p}_{1} \tilde{q}_{1}}{\varepsilon \tilde{t}}, \\
& q_{1}=\frac{\tilde{t} \tilde{p}_{1}}{\tilde{p}_{1} \tilde{q}_{1}+\theta_{2}^{\infty},} \quad p_{1}=-\frac{\tilde{q}_{1}}{\tilde{t}}\left(\tilde{p}_{1} \tilde{q}_{1}+\theta_{2}^{\infty}\right), \quad q_{2}=-\varepsilon \tilde{q}_{2}, \quad p_{2}=-\varepsilon^{-1} \tilde{p}_{2}, \\
& x=\varepsilon^{-1} \tilde{x}, \quad Y=\left(\begin{array}{ccc}
1 & 0 & 0 \\
0 & -1 & 0 \\
0 & 0 & -1 / t
\end{array}\right) \tilde{Y} .
\end{aligned}
$$

$3 / 2+1+1 \rightarrow 4 / 3+1+1$

$(1)_{2} 1,21,111 \rightarrow(1)_{3}, 21,111$

$$
\begin{aligned}
& \theta_{1}^{\infty}=\tilde{\theta}_{1}^{\infty}-\varepsilon^{-1}, \quad \theta_{2}^{\infty}=\varepsilon^{-1}, \quad t=\varepsilon \tilde{t}, \quad H=\varepsilon^{-1} \tilde{H}-\frac{\tilde{p}_{1} \tilde{q}_{1}}{\varepsilon \tilde{t}}, \quad q_{1}=\varepsilon \tilde{q}_{2}, \\
& p_{1}=\varepsilon^{-1} \tilde{p}_{2}, \quad q_{2}=\frac{\tilde{t}}{\tilde{q}_{1}}, \quad p_{2}=-\frac{\tilde{q}_{1}}{\tilde{t}}\left(\tilde{p}_{1} \tilde{q}_{1}-\theta_{1}^{0}+\theta_{2}^{0}\right), \quad Y=\left(\begin{array}{ccc}
t & 0 & 0 \\
0 & 1 & 0 \\
0 & 0 & -q_{2} / t
\end{array}\right) \tilde{Y} .
\end{aligned}
$$

$\mathbf{2}+\mathbf{3} / \mathbf{2} \rightarrow \mathbf{3} / \mathbf{2}+\mathbf{3} / \mathbf{2}$

$(1)_{2} 1,(11)(1) \rightarrow(1)_{2} 1,(1)_{2} 1$

$$
\begin{aligned}
& \theta_{1}^{0}=\tilde{\theta}^{0}+\varepsilon^{-1}, \quad \theta_{2}^{0}=-\varepsilon^{-1}, \quad t=\varepsilon \tilde{t}, \quad H=\varepsilon^{-1} \tilde{H}-\frac{\tilde{p}_{2} \tilde{q}_{2}}{\varepsilon \tilde{t}}, \\
& q_{1}=-\varepsilon \tilde{t} \tilde{p}_{2}, \quad p_{1}=\frac{\tilde{q}_{2}}{\varepsilon \tilde{t}}-\frac{1}{\varepsilon \tilde{q}_{1}}, \quad q_{2}=\tilde{q}_{1}, \quad p_{2}=\tilde{p}_{1}+\frac{-\theta_{2}^{\infty}+\varepsilon^{-1}}{\tilde{q}_{1}}-\frac{\tilde{t} \tilde{p}_{2}}{\tilde{q}_{1}^{2}}, \\
& x=\varepsilon^{-1} \tilde{x}, \quad Y=t^{-\theta_{1}^{\infty}}\left(\begin{array}{ccc}
1 & 0 & 0 \\
0 & 1 & 0 \\
0 & 0 & q_{2}
\end{array}\right) \tilde{Y} .
\end{aligned}
$$

$\mathbf{2}+\mathbf{3} / \mathbf{2} \rightarrow \mathbf{2}+\mathbf{4} / \mathbf{3}$

$(1)_{2} 1,(11)(1) \rightarrow(1)_{3},(11)(1)$

$$
\begin{aligned}
& \theta_{1}^{\infty}=\tilde{\theta}_{1}^{\infty}-\varepsilon^{-1}, \quad \theta_{2}^{\infty}=\varepsilon^{-1}, \quad \theta_{1}^{0}=\tilde{\theta}_{2}^{0}, \quad \theta_{2}^{0}=\tilde{\theta}_{1}^{0}, \quad q_{1}=-\varepsilon \tilde{t} \tilde{p}_{2}, \quad p_{1}=\frac{\tilde{q}_{2}}{\varepsilon \tilde{t}}, \\
& q_{2}=\tilde{q}_{1}, \quad p_{2}=\tilde{p}_{1}, \quad t=\varepsilon \tilde{t}, \quad H=\varepsilon^{-1} \tilde{H}-\frac{\tilde{p}_{2} \tilde{q}_{2}}{\varepsilon \tilde{t}}, \quad Y=\left(\begin{array}{ccc}
t & 0 & 0 \\
0 & 1 & 0 \\
0 & 0 & 1
\end{array}\right) \tilde{Y} .
\end{aligned}
$$

$4 / 3+1+1 \rightarrow 2+4 / 3$

$(1)_{3}, 21,111 \rightarrow(1)_{3},(11)(1)$

$$
\begin{aligned}
& \theta^{1}=-\varepsilon^{-1}, \quad \theta_{2}^{0}=\tilde{\theta}_{2}^{0}+\varepsilon^{-1}, \quad q_{1}=-\varepsilon \tilde{t} \tilde{p}_{1}, \quad p_{1}=\frac{\tilde{q}_{1}}{\varepsilon \tilde{t}}, \quad t=\varepsilon \tilde{t}, \\
& H=\varepsilon^{-1} \tilde{H}-\frac{\tilde{p}_{1} \tilde{q}_{1}}{\varepsilon \tilde{t}}, \quad x=-\varepsilon^{-1} \tilde{x}, \quad Y=\left(\begin{array}{ccc}
1 & 0 & 0 \\
0 & -\varepsilon & 0 \\
0 & -\varepsilon & \frac{\varepsilon q_{1}}{t}
\end{array}\right) \tilde{Y} .
\end{aligned}
$$


$3 / 2+3 / 2 \rightarrow 3 / 2+4 / 3$

$(1)_{2} 1,(1)_{2} 1 \rightarrow(1)_{3},(1)_{2} 1$

$$
\begin{aligned}
& \theta_{1}^{\infty}=-\varepsilon^{-1}, \quad \theta_{2}^{\infty}=\tilde{\theta}_{1}^{\infty}+\varepsilon, \quad t=\varepsilon \tilde{t}, \quad H=\varepsilon^{-1} \tilde{H}, \\
& q_{1}=\tilde{q}_{1}, \quad p_{1}=\tilde{p}_{1}+\frac{\tilde{\theta}_{1}^{\infty}+\varepsilon^{-1}}{\tilde{q}_{1}}, \quad Y=t^{-\varepsilon^{-1}}\left(\begin{array}{ccc}
t & 0 & 0 \\
0 & 1 & 0 \\
0 & 0 & 1
\end{array}\right) \tilde{Y} .
\end{aligned}
$$

$2+4 / 3 \rightarrow 3 / 2+4 / 3$

$(1)_{3},(11)(1) \rightarrow(1)_{3},(1)_{2} 1$

$$
\begin{aligned}
& \theta_{1}^{0}=\tilde{\theta}^{0}-\varepsilon^{-1}, \quad \theta_{2}^{0}=\varepsilon^{-1}, \quad t=\varepsilon \tilde{t}, \quad H=\varepsilon^{-1} \tilde{H}, \\
& q_{1}=\tilde{q}_{1}, \quad p_{1}=\tilde{p}_{1}+\frac{-\tilde{\theta}^{0}+\varepsilon^{-1}}{\tilde{q}_{1}}, \quad x=\varepsilon^{-1} \tilde{x}, \quad Y=\left(\begin{array}{ccc}
1 & 0 & 0 \\
0 & \varepsilon & 0 \\
0 & 0 & \varepsilon q_{1}
\end{array}\right) \tilde{Y} .
\end{aligned}
$$

$3 / 2+4 / 3 \rightarrow 4 / 3+4 / 3$

$(1)_{3},(1)_{2} 1 \rightarrow(1)_{3},(1)_{3}$

$$
\begin{array}{ll}
\theta^{0}=3 \varepsilon^{-1}, & \theta_{1}^{\infty}=-3 \varepsilon^{-1}, \quad t=\varepsilon \tilde{t}, \quad H=\varepsilon \tilde{H}-\frac{\tilde{p}_{1} \tilde{q}_{1}+\tilde{p}_{2} \tilde{q}_{2}}{\varepsilon \tilde{t}}, \\
q_{1}=-\frac{\tilde{t}}{\tilde{q}_{1}}, & p_{1}=\frac{\tilde{q}_{1}}{\tilde{t}}\left(\tilde{p}_{1} \tilde{q}_{1}+\frac{1}{3}-\frac{1}{\varepsilon}\right), \quad q_{2}=-\frac{\tilde{t}}{\tilde{q}_{2}}, \quad p_{2}=\frac{\tilde{q}_{2}}{\tilde{t}}\left(\tilde{p}_{2} \tilde{q}_{2}+\frac{2}{3}+\frac{1}{\varepsilon}\right), \\
x=\varepsilon^{-1} \tilde{x}, & Y=t^{\frac{1}{\varepsilon}-\frac{1}{3}} x^{\frac{1}{\varepsilon}-\frac{1}{3}}\left(\begin{array}{ccc}
1 & 0 & 0 \\
0 & \varepsilon & 0 \\
0 & 0 & t / q_{1}
\end{array}\right) \tilde{Y} .
\end{array}
$$

\section{Acknowledgements}

The author would like to thank Professor Kazuki Hiroe for his helpful suggestions. The author would also like to thank Professors Hidetaka Sakai and Akane Nakamura who provided invaluable comments and continual encouragement.

\section{References}

[1] Babbitt D.G., Varadarajan V.S., Formal reduction theory of meromorphic differential equations: a group theoretic view, Pacific J. Math. 109 (1983), 1-80.

[2] Boalch P., Simply-laced isomonodromy systems, Publ. Math. Inst. Hautes Études Sci. 116 (2012), 1-68, arXiv:1107.0874.

[3] Fuji K., Suzuki T., Drinfeld-Sokolov hierarchies of type $A$ and fourth order Painlevé systems, Funkcial. Ekvac. 53 (2010), 143-167, arXiv:0904.3434.

[4] Gambier B., Sur les équations différentielles du second ordre et du premier degré dont l'intégrale générale est a points critiques fixes, Acta Math. 33 (1910), 1-55.

[5] Garnier R., Sur des équations différentielles du troisième ordre dont l'intégrale générale est uniforme et sur une classe d'équations nouvelles d'ordre supérieur dont l'intégrale générale a ses points critiques fixes, Ann. Sci. École Norm. Sup. (3) 29 (1912), 1-126.

[6] Harnad J., Dual isomonodromic deformations and moment maps to loop algebras, Comm. Math. Phys. 166 (1994), 337-365, hep-th/9301076.

[7] Hiroe K., Oshima T., A classification of roots of symmetric Kac-Moody root systems and its application, in Symmetries, Integrable Systems and Representations, Springer Proc. Math. Stat., Vol. 40, Springer, Heidelberg, 2013, 195-241. 
[8] Hukuhara M., Sur les points singuliers des équations différentielles linéaires, II, J. Fac. Sci. Hokkaido Univ. 5 (1937), 123-166.

[9] Jimbo M., Miwa T., Ueno K., Monodromy preserving deformation of linear ordinary differential equations with rational coefficients. I. General theory and $\tau$-function, Phys. D 2 (1981), 306-352.

[10] Kapaev A.A., Lax pairs for Painlevé equations, in Isomonodromic Deformations and Applications in Physics (Montréal, QC, 2000), CRM Proc. Lecture Notes, Vol. 31, Amer. Math. Soc., Providence, RI, 2002, $37-48$.

[11] Kapaev A.A., Hubert E., A note on the Lax pairs for Painlevé equations, J. Phys. A: Math. Gen. 32 (1999), 8145-8156.

[12] Kawakami H., Matrix Painlevé systems, J. Math. Phys. 56 (2015), 033503, 27 pages.

[13] Kawakami H., Four-dimensional Painlevé-type equations associated with ramified linear equations I: Matrix Painlevé systems, arXiv:1608.03927.

[14] Kawakami H., Four-dimensional Painlevé-type equations associated with ramified linear equations II: Sasano systems, arXiv:1609.05263.

[15] Kawakami H., Nakamura A., Sakai H., Degeneration scheme of 4-dimensional Painlevé-type equations, arXiv:1209.3836.

[16] Kawamuko H., On the Garnier system of half-integer type in two variables, Funkcial. Ekvac. 52 (2009), $181-201$.

[17] Kimura H., The degeneration of the two-dimensional Garnier system and the polynomial Hamiltonian structure, Ann. Mat. Pura Appl. 155 (1989), 25-74.

[18] Levelt A.H.M., Jordan decomposition for a class of singular differential operators, Ark. Mat. 13 (1975), $1-27$.

[19] Nakamura A., Autonomous limit of 4-dimensional Painlevé-type equations and degeneration of curves of genus two, arXiv:1505.00885.

[20] Ohyama Y., Kawamuko H., Sakai H., Okamoto K., Studies on the Painlevé equations. V. Third Painlevé equations of special type $P_{\mathrm{III}}\left(D_{7}\right)$ and $P_{\mathrm{III}}\left(D_{8}\right)$, J. Math. Sci. Univ. Tokyo 13 (2006), 145-204.

[21] Ohyama Y., Okumura S., A coalescent diagram of the Painlevé equations from the viewpoint of isomonodromic deformations, J. Phys. A: Math. Gen. 39 (2006), 12129-12151.

[22] Okamoto K., Studies on the Painlevé equations. III. Second and fourth Painlevé equations, $P_{\mathrm{II}}$ and $P_{\mathrm{IV}}$, Math. Ann. 275 (1986), 221-255.

[23] Okamoto K., Studies on the Painlevé equations. I. Sixth Painlevé equation $P_{\mathrm{VI}}$, Ann. Mat. Pura Appl. 146 (1987), 337-381.

[24] Okamoto K., Studies on the Painlevé equations. II. Fifth Painlevé equation P. Japan. J. Math. (N.S.) 13 (1987), 47-76.

[25] Okamoto K., Studies on the Painlevé equations. IV. Third Painlevé equation PIII, Funkcial. Ekvac. 30 (1987), 305-332.

[26] Painlevé P., Sur les équations différentielles du second ordre à points critiques fixes, C. R. Acad. Sci. Paris 127 (1898), 945-948.

[27] Sakai H., Rational surfaces associated with affine root systems and geometry of the Painlevé equations, Comm. Math. Phys. 220 (2001), 165-229.

[28] Sakai H., Isomonodromic deformation and 4-dimensional Painlevé type equations, Preprint, University of Tokyo, Mathematical Sciences, 2010.

[29] Sasano Y., Coupled Painlevé VI systems in dimension four with affine Weyl group symmetry of type $D_{6}^{(1)}$. II, in Algebraic Analysis and the Exact WKB Analysis for Systems of Differential Equations, RIMS Kôkyûroku Bessatsu, B5, Res. Inst. Math. Sci. (RIMS), Kyoto, 2008, 137-152, arXiv:0704.2367.

[30] Suzuki T., A particular solution of a Painlevé system in terms of the hypergeometric function ${ }_{n+1} F_{n}, S I G M A$ 6 (2010), 078, 11 pages, arXiv:1004.0059.

[31] Tsuda T., UC hierarchy and monodromy preserving deformation, J. Reine Angew. Math. 690 (2014), 1-34, arXiv:1007.3450.

[32] Turrittin H.L., Convergent solutions of ordinary linear homogeneous differential equations in the neighborhood of an irregular singular point, Acta Math. 93 (1955), 27-66.

[33] Wasow W., Asymptotic expansions for ordinary differential equations, Pure and Applied Mathematics, Vol. 14, Interscience Publishers John Wiley \& Sons, Inc., New York - London - Sydney, 1965.

[34] Yamakawa D., Middle convolution and Harnad duality, Math. Ann. 349 (2011), 215-262, arXiv:0911.3863. 